\title{
Colloquium: Nonthermal pathways to ultrafast control in quantum materials
}

\author{
Alberto de la Torre $\odot^{*}$ \\ Department of Physics, Brown University, Providence, Rhode Island 02912, USA \\ Dante M. Kennes $\odot^{\dagger}$ \\ Institut für Theorie der Statistischen Physik, RWTH Aachen University \\ and JARA Fundamentals of Future Information Technology, 52056 Aachen, Germany \\ and Max Planck Institute for the Structure and Dynamics of Matter, \\ Center for Free-Electron Laser Science (CFEL), \\ Luruper Chaussee 149, 22761 Hamburg, Germany
}

Martin Claassen

Department of Physics and Astronomy, University of Pennsylvania, Philadelphia, Pennsylvania 19104, USA

Simon Gerber $\circledast^{\S}$

Laboratory for Micro and Nanotechnology, Paul Scherrer Institut, Forschungsstrasse 111, CH-5232 Villigen PSI, Switzerland

James W. Mclverøी and Michael A. Sentef $\oplus^{\|}$

Max Planck Institute for the Structure and Dynamics of Matter, Center for Free-Electron Laser Science (CFEL), Luruper Chaussee 149, 22761 Hamburg, Germany

(published 14 October 2021)

Recent progress in utilizing ultrafast light-matter interaction to control the macroscopic properties of quantum materials is reviewed. Particular emphasis is placed on photoinduced phenomena that do not result from ultrafast heating effects but rather emerge from microscopic processes that are inherently nonthermal in nature. Many of these processes can be described as transient modifications to the free energy landscape resulting from the redistribution of quasiparticle populations, the dynamical modification of coupling strengths, and the resonant driving of the crystal lattice. Other pathways result from the coherent dressing of a material's quantum states by the light field. A selection of recently discovered effects leveraging these mechanisms, as well as the technological advances that led to their discovery, is discussed. A road map for how the field can harness these nonthermal pathways to create new functionalities is presented.

DOI: 10.1103/RevModPhys.93.041002

\section{CONTENTS}

I. Introduction

II. Emergent Phenomena after Pulsed Laser Excitation

A. Weak-excitation regime: Collective modes and disentangling degrees of freedom

B. Optical switching

\footnotetext{
*alberto_de_la_torre_duran@brown.edu

†dante.kennes@rwth-aachen.de

‡claassen@sas.upenn.edu

simon.gerber@psi.ch

†james.mciver@mpsd.mpg.de

"michael.sentef@mpsd.mpg.de
}

C. Out-of-equilibrium critical behavior 6

D. Nonlinearities and dynamical couplings $\quad 7$

1. Dynamical Hubbard $U$ interaction $\quad 7$

2. Nonlinear phononics 8

III. Dressed States of Nonequilibrium Matter 9

A. Theory introduction $\quad 10$

1. Dynamical localization in a driven atomic chain 10

2. Floquet primer

B. Dressed band structures:

Floquet topological insulators

C. Toward Floquet many-body physics: Heating and interactions

D. Engineering correlated sys

IV. Outlook

Acknowledgments $\quad 17$ 
Appendix: Technical Advances Shaping Ultrafast Quantum Materials Science

1. Experimental tools

2. Theoretical tools

References

\section{INTRODUCTION}

Quantum materials host a wide range of many-body and topological phenomena that both challenge our physical understanding of solids and offer possibilities for nextgeneration technologies. From unconventional superconductivity to topologically protected edge modes, the remarkable physics in quantum materials emerges from complex interactions between spin, charge, lattice, and orbital degrees of freedom (Keimer and Moore, 2017) and the geometric and topological aspects of their wave functions (Wang and Zhang, 2017; Narang, Garcia, and Felser, 2021). Many materials host multiple quantum phases that can be independently accessed by the application of external perturbations such as electromagnetic fields, pressure, strain, and chemical doping, making these systems ideal platforms for future technological applications (Tokura, Kawasaki, and Nagaosa, 2017). Moreover, the search for new ways to create and control their macroscopic properties continues to improve our fundamental understanding of the interactions between the underlying degrees of freedom, which in turn may lead to new functionalities (Basov, Averitt, and Hsieh, 2017; Alexandradinata et al., 2020; Giustino et al., 2021).

One promising route to controlling and understanding quantum materials is ultrafast light-matter interaction. This can induce long-lived nonequilibrium states with functionally relevant properties that cannot be realized in thermal equilibrium. Since the pioneering work of Koshihara et al. (1990) on photoinduced phase transitions, ultrafast optical experiments have been used to investigate a variety of nonequilibrium effects in quantum materials. Breakthrough results in the past decade include the surgical decoupling of microscopic degrees of freedom in iron-based superconductors (Gerber et al., 2017), ultrafast switching into hidden phases in transition-metal dichalcogenides (Stojchevska et al., 2014), dynamically controlled microscopic interactions in correlated transition-metal oxides (Först, Manzoni et al., 2011), possible light-induced superconductivity in organic compounds (Mitrano et al., 2016), and photon-dressed topological states in topological insulators (Wang et al., 2013) and graphene (McIver et al., 2020).

The mechanisms behind many of the most intriguing nonequilibrium phenomena go beyond the simple melting of thermal states by laser-induced heating. They rely on distinct nonthermal pathways, such as transient modifications to the free energy landscape or photon dressing effects, where

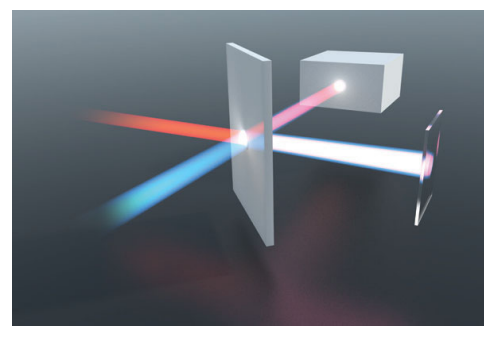

Optical probes

- Probes dielectric properties

- Flexible in implementation (spectral range, detection scheme, environment) - fs time resolution

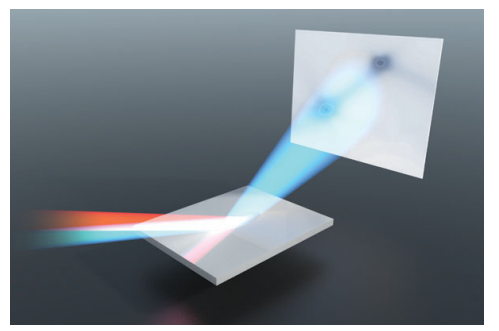

Scattering probes

- Probes structural dynamics and dynamics of electronic degrees of freedom at elemental resonances

- Access to dispersion relations via finite momentum transfer

- fs time resolution
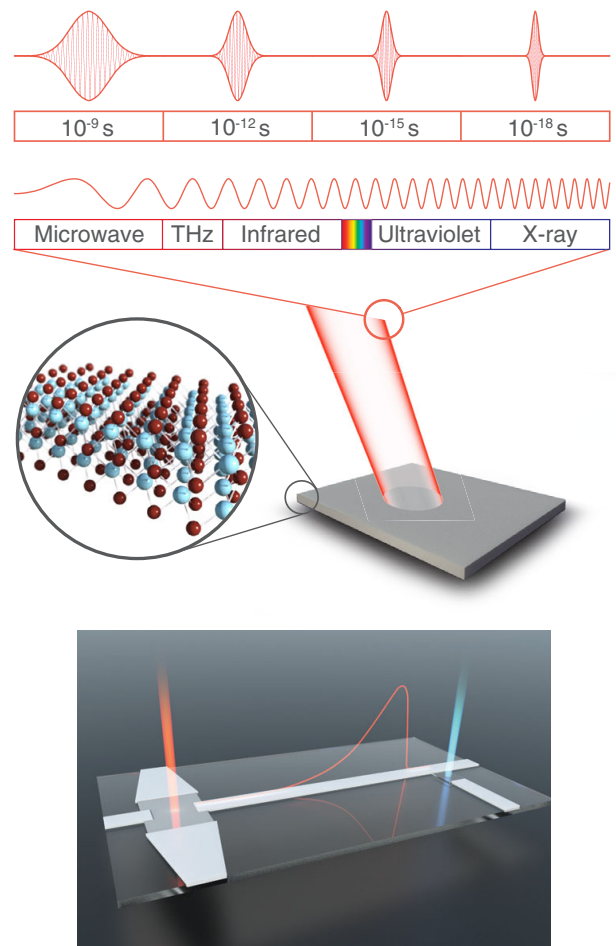

Transport

- Probes transient photoconductivity

- Integrates well into microstructured devices

- Sub-ps time resolution

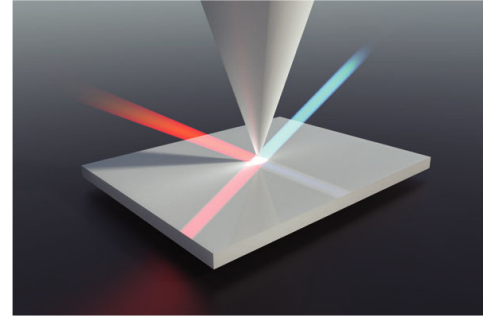

Scanning probes

- Probes optical constants in near-field (SNOM) or tunneling currents (STM) - fs time resolution

- nm spatial resolution

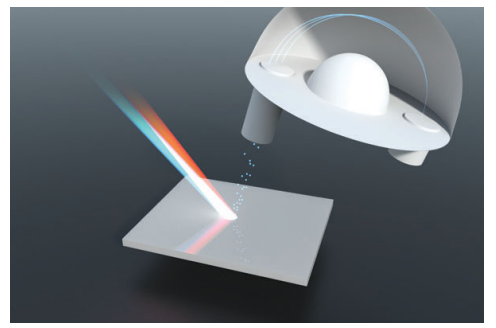

ARPES

- Probes time- and momentum-resolved carrier dynamics, and the evolution of electronic spectral functions

- Direct probe of electronic temperature - Tunability of energy vs. time resolution (down to $\sim 15 \mathrm{meV}, \sim 30 \mathrm{fs}$ )

FIG. 1. Overview of the main experimental tools available to investigate ultrafast phenomena in quantum materials. The upper central panel illustrates the broad spectral and temporal ranges from which tailored short laser pulses can be created to photoexcite (pump) materials out of equilibrium. The surrounding panels show categories of ultrafast time-resolved probes and their capabilities. 


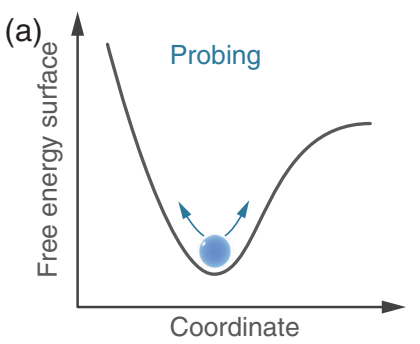

(b)

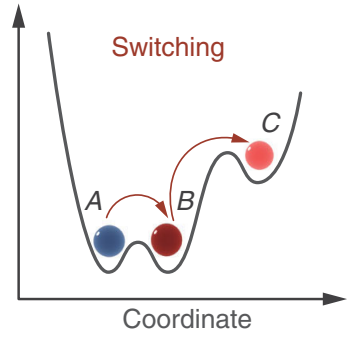

(c)

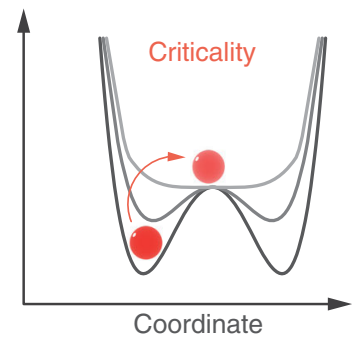

(d)

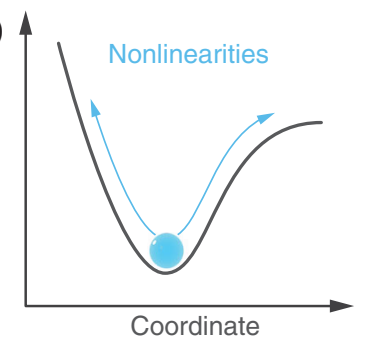

FIG. 2. Illustration of nonthermal pathways triggered by laser excitation. All panels show a potential or free energy landscape as a function of a system coordinate, such as an electronic order parameter or a lattice displacement. (a) Weak excitations around a stable minimum (ground state) permit one to probe collective modes and their mutual couplings. (b) With a short, more intense laser pulse, one can switch between degenerate ground states $(A$ and $B$ ) or drive the material to a nonthermal, metastable excited state $C$. (c) Nonequilibrium critical behavior can be induced by a short, even more intense excitation that transiently modifies the free energy landscape itself. (d) With a strong excitation, the system can also be driven into anharmonic regimes with deviations from parabolic behavior, which allows one to probe nonlinear effects and change effective couplings in the material.

quasithermal descriptions based on effective temperatures are insufficient or impossible.

Here we survey recent efforts in applying nonthermality as a resource to exert control over quantum materials on ultrashort timescales in a flexible and reversible manner. We highlight a selection of phenomena that emerge after pulsed laser excitation, including nonthermal switching mechanisms, out-of-equilibrium critical behavior, and the nonlinear control of microscopic couplings and phononics (Sec. II). Another class of nonthermal phenomena are those that occur for the duration of the laser pulse, where the microscopic degrees of freedom are strongly coupled to the amplitude, frequency, and polarization of the light field, in some cases forming dressed states that can be described by Floquet theory. We review recent realizations of Floquet engineering protocols in quantum materials and discuss new theoretical directions for future experiments (Sec. III).

Recent progress in the field is due in part to the development of improved time-resolved experimental techniques. State-of-the-art light sources can now reliably generate intense optical pulses at wavelengths spanning from the terahertz to the extreme ultraviolet with a wide range of available pulse durations and repetition rates (Steinmeyer et al., 1999; Bartels et al., 2002; Cerullo and De Silvestri, 2003; Reimann, 2007; Liu et al., 2017). These advances have enabled a surgical approach to photoexciting ("pumping") quantum materials in pump-probe experiments, for example, by selectively accessing electronic transitions, directly coupling to collective modes, or intentionally avoiding such resonances entirely. This enhanced pump tunability, combined with a fleet of experimental probes targeting complementary macroscopic observables (Fig. 1 and Appendix A.1), has led to an improved understanding of nonequilibrium phenomena in solids and the nonthermal pathways leading to their creation. Moreover, these results have triggered complementary theoretical efforts toward modeling microscopic dynamics in photoexcited quantum materials (Appendix A.2).

A full-fledged review of the rapidly growing field of ultrafast quantum materials science is beyond the scope of this Colloquium. To account for the important early works, we refer to other reviews, including those by Averitt and Taylor (2002), Basov et al. (2011), Orenstein (2012), Zhang and Averitt (2014), and Giannetti et al. (2016), which provide extensive reports of ultrafast spectroscopy of strongly correlated electron systems. Kirilyuk, Kimel, and Rasing (2010) wrote an in-depth review on ultrafast magneto-optical effects. Buzzi et al. (2018) reviewed ultrafast structural dynamics in solids probed by time-resolved $\mathrm{x}$-ray scattering. Overviews of time-resolved inelastic scattering $\mathrm{x}$-ray scattering were provided by Cao et al. (2019) and Mitrano and Wang (2020). Further reviews on Floquet engineering were given by Bukov, D'Alessio, and Polkovnikov (2015), Oka and Kitamura (2019), and Rudner and Lindner (2020).

\section{EMERGENT PHENOMENA AFTER PULSED LASER EXCITATION}

We discuss here emergent phenomena in quantum materials occurring after photoexcitation by an ultrafast laser pulse. In particular, we focus on processes dominated by dynamics that cannot be described by a hot electronic subsystem that thermalizes back to equilibrium through heat exchange with the cold crystalline lattice (phonons), the so-called two-temperature models (Anisimov, Kapeliovich, and Perelman, 1974; Allen, 1987) or $n$-temperature generalizations (Koopmans et al., 2010). These descriptions rest on the assumption that the mutual couplings between degrees of freedom are not modified by the excitation (Allen, 1987; Petek and Ogawa, 1997; Bauer, Marienfeld, and Aeschlimann, 2015). In quantum materials, this is not necessarily the case, and the optical excitation can result in modified or suppressed coupling constants (Ishioka et al., 2008; Murakami et al., 2015). These simplified models neglect nonthermal effects resulting from ultrafast light-matter coupling. For example, in many correlated systems the notion of thermality, in the sense of a unique thermal density matrix and temperature, breaks down on ultrashort (femtosecond) to intermediate (subpicosecond) timescales (Kemper, Abdurazakov, and Freericks, 2018). This departure from the thermal response makes the pulsed excitation of quantum materials a unique strategy for creating and controlling quantum phenomena.

We depict in Fig. 2 different scenarios of transient phenomena in terms of a simplified free energy landscape as a function of growing excitation strength. In the weak-excitation regime, the optical drive can excite collective oscillations of the underlying order parameters [Fig. 2(a), Sec. II.A]. By tracking 
these modes in the time domain one can disentangle microscopic degrees of freedom and their mutual couplings. In some materials, photoexcitation can drive the system into a different low-energy state or hidden states not accessible in thermal equilibrium [Fig. 2(b), Sec. II.B]. Under the right excitation conditions, ultrafast transient modifications of the free energy landscape can also induce nonequilibrium phase transitions and enable the study of nonthermal critical behavior [Fig. 2(c), Sec. II.C]. Finally, strong excitations can drive a system into nonlinear regimes, dynamically modifying the effective microscopic couplings governing its quantum many-body wave function, thus providing another strategy to investigate emergent phenomena with no equilibrium counterpart [Fig. 2(d), Sec. II.D].

\section{A. Weak-excitation regime: Collective modes and disentangling degrees of freedom}

We consider a situation where the system under investigation is only weakly driven; i.e., it remains in a linear regime [Fig. 2(a)]. The recovery toward equilibrium is sensitive to collective modes and reflects the characteristic timescales of the different degrees of freedom. Prominent examples of coherent modes in ordered states that have been probed using time-domain techniques are amplitude modes of charge-density wave materials (Demsar, Biljaković, and Mihailovic, 1999; Demsar et al., 2002; Schmitt et al., 2008; Lee et al., 2012; Mihailovic, 2019; Zong et al., 2019a), potential excitonic insulators (Werdehausen et al., 2018), and the Higgs amplitude (Matsunaga et al., 2013, 2014; Chu et al., 2020) and Leggett modes (Giorgianni et al., 2019), as well as Josephson plasmons (Laplace and Cavalleri, 2016) in superconductors.

In the weak-excitation regime, charge, lattice, spin, or orbital degrees of freedom of correlated electron systems can be disentangled in the time domain by an appropriate choice of the experimental probe [Fig. 3(a)]. Under particular circumstances these techniques can be brought together in a unified fashion, as demonstrated by Gerber et al. (2017), who used time- and angle-resolved photoemission spectroscopy (tr-ARPES) and x-ray scattering to study the significance of a cooperative interplay among electron-electron and electronphonon interactions in the iron-based superconductor parent compound FeSe. Figure 3(b) depicts how both the lattice displacement and the electronic band structure lock into a coherent $A_{1 g}$ phonon mode that is driven by an ultrafast infrared (IR) laser pulse. In turn, this terahertz frequency locking of the 2 degrees of freedom allows for a purely experimental, highly precise quantification of fundamental physical properties. For example, for FeSe it was used to quantify the orbitally resolved electron-phonon deformation potential, which reveals the critical importance of electron correlations for the material's properties.

This example shows that such multimessenger approaches allow one not only to infer nonequilibrium characteristics but also to extract equilibrium properties via the time domain (Gerber et al., 2017; Mitrano et al., 2019). A caveat concerns the difficulty in bringing together more than one ultrafast experiment in a unified fashion. Ideally, a multimessenger experiment is conducted in one single setup, such as that used
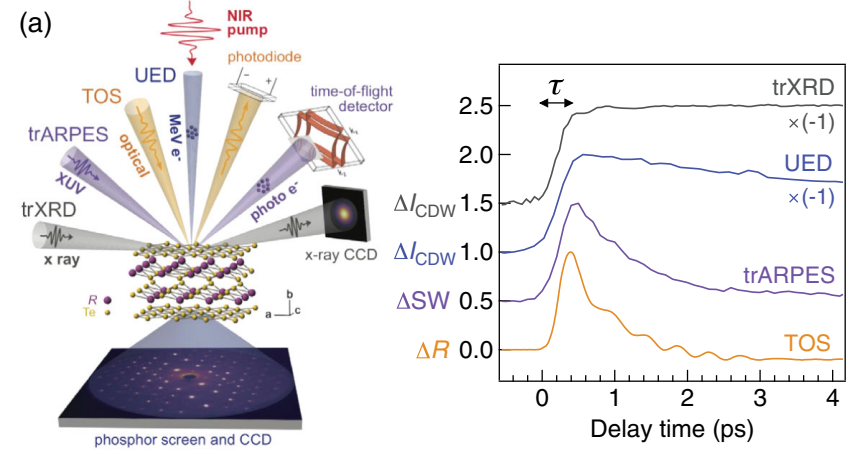

(b)

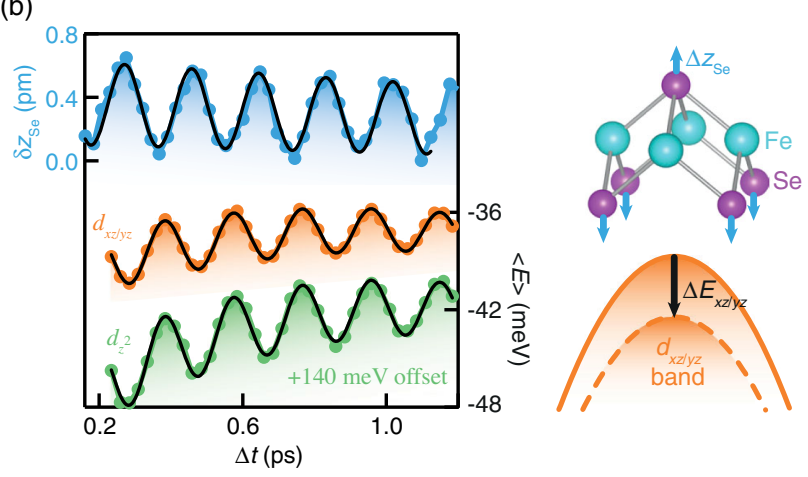

FIG. 3. Multimessenger probing of disentangled microscopic degrees of freedom. (a) A portfolio of ultrafast techniques has been used to investigate photoinduced melting of charge-density wave order in the materials $(\mathrm{La} / \mathrm{Tb}) \mathrm{Te}_{3}$, including time-resolved $\mathrm{x}$-ray diffraction (tr-XRD), time- and angle-resolved photoemission spectroscopy (tr-ARPES), transient optical spectroscopy (TOS), and ultrafast electron diffraction (UED). The signals obtained can be qualitatively and quantitatively different since distinct degrees of freedom are probed. From Zong et al., 2019a. (b) Se atoms in FeSe are coherently displaced following photoexcitation by an ultrafast IR pulse, which can be directly measured by tr-XRD (blue, top). At the same time, corresponding energy shifts of electron bands are revealed by tr-ARPES (orange, middle; green, bottom). The schematic on the right depicts the $A_{1 g}$ phonon mode that induces the periodic modulation of the lattice and electronic bands. From Gerber et al., 2017.

for the simultaneous detection of electronic and structural (Porer et al., 2014; Mardegan et al., 2021) or multiple structural orders (Morrison et al., 2014; Kogar et al., 2020; Zhou et al., 2021). This ensures that the pumping conditions, such as pump fluence, pump and probe beam profile, time resolution, penetration depth (effectively absorbed energy densities), and, notably, also the definition of time zero (the instant when the pump pulse excites the sample) are guaranteed to be consistent. Such unified experimental setups allow one to compare not only the amplitude signals of the disentangled degrees of freedom but also their phase relation and decay rates (dephasing times), paving the way to coherent quantum control of (dis)entangled degrees of freedom.

\section{B. Optical switching}

If one goes one step further from equilibrium, a natural question to ask is whether nonthermal pathways can be found to photoinduce a phase transition, that is, optically switch the 
system into parts of the free energy landscape that are not accessible in thermal equilibrium. Indeed, the key idea behind optically driven switching is similar to basic concepts of chemical reaction pathways, in which catalysts help the system overcome an activation energy barrier in order to trigger the intended reaction.

We start with a pedagogical description within a free energy landscape framework, as sketched in Fig. 2(b) (Nasu, Ping, and Mizouchi, 2001). We consider a long-range ordered symmetry-broken ground state given by one of the global minima of the free energy landscape, which is indicated by $A$. To reach any of the local minima in its vicinity $(C)$ the system has to overcome an associated energy cost that might be insurmountable in thermal equilibrium. However, excitation with an ultrashort laser pulse can lift the system over this energy barrier by nonadiabatic energy transfer into the electronic degrees of freedom and give rise to metastable phases (Koshihara et al., 1990; Tokura, 2006), which in some cases can persist indefinitely on experimental timescales, as first reported in manganites (Kiryukhin et al., 1997; Miyano et al., 1997). In $1 T-\mathrm{TaS}_{2}$ (Fazekas and Tosatti, 1979), the ultrafast transfer of energy into the electronic degrees of freedom by either laser ( $<35 \mathrm{fs)} \mathrm{(Perfetti} \mathrm{et} \mathrm{al.,} \mathrm{2006;}$ Stojchevska et al., 2014; Zong et al., 2018; Gerasimenko et al., 2019; Vodeb et al., 2019; Danz, Domröse, and Ropers, 2021) or electrical ( 30 ps) pulses (Vaskivskyi et al., 2015; Mraz et al., 2021) results in a long-lived amorphouslike metallic state, as illustrated in Fig. 4(a), due to the formation of light-induced topological defects (Haupt et al., 2016; Laulhé et al., 2017). Another example of an ultrafast transition induced by nonthermal electronic configurations is the switching into long-lived high-symmetry crystalline phases in prototypical Peierls systems, as shown by Huber et al. (2014), Teitelbaum et al. (2018), and Naseska et al. (2021) [Fig. 4(b)]. Ultrafast excitation of the electronic degrees of freedom in these materials results in a nonthermal distribution that thermalizes into the high-energy metastable state via dissipation of the surplus energy into a heat bath, e.g., phonons or the substrate (Demsar et al., 2004). The equilibrium state is then reinstated after a slow relaxation process or upon heating above a critical temperature (Vaskivskyi et al., 2015; Gerasimenko et al., 2019). For a comprehensive theoretical description of ultrafast optical switching in terms of a Ginzburg-Landau (GL) theory see the recent work by Sun and Millis (2020).

Nonthermal metastable phases provide new opportunities for controllable and reversible switching and thus have potential for technological applications. This has inspired work in a variety of quantum materials families. To exemplify the universality of optical switching and to acknowledge their relevance within the ultrafast condensed matter field, we highlight here the three following research directions: (i) lightinduced insulator-to-metal transitions in nanofabricated (Liu et al., 2012) and pristine samples of the correlated insulator $\mathrm{VO}_{2}$ (Becker et al., 1994; Cavalleri et al., 2001, 2004; Baum, Yang, and Zewail, 2007; Morrison et al., 2014; Wegkamp et al., 2014; Wall et al., 2018; Otto et al., 2019), (ii) the control of the spin-orbit-lattice-coupled ground state in manganites (Tokura and Nagaosa, 2000) by either laser (Takubo et al., 2005; Ichikawa et al., 2011; Li et al., 2013, 2018; Beaud et al.,
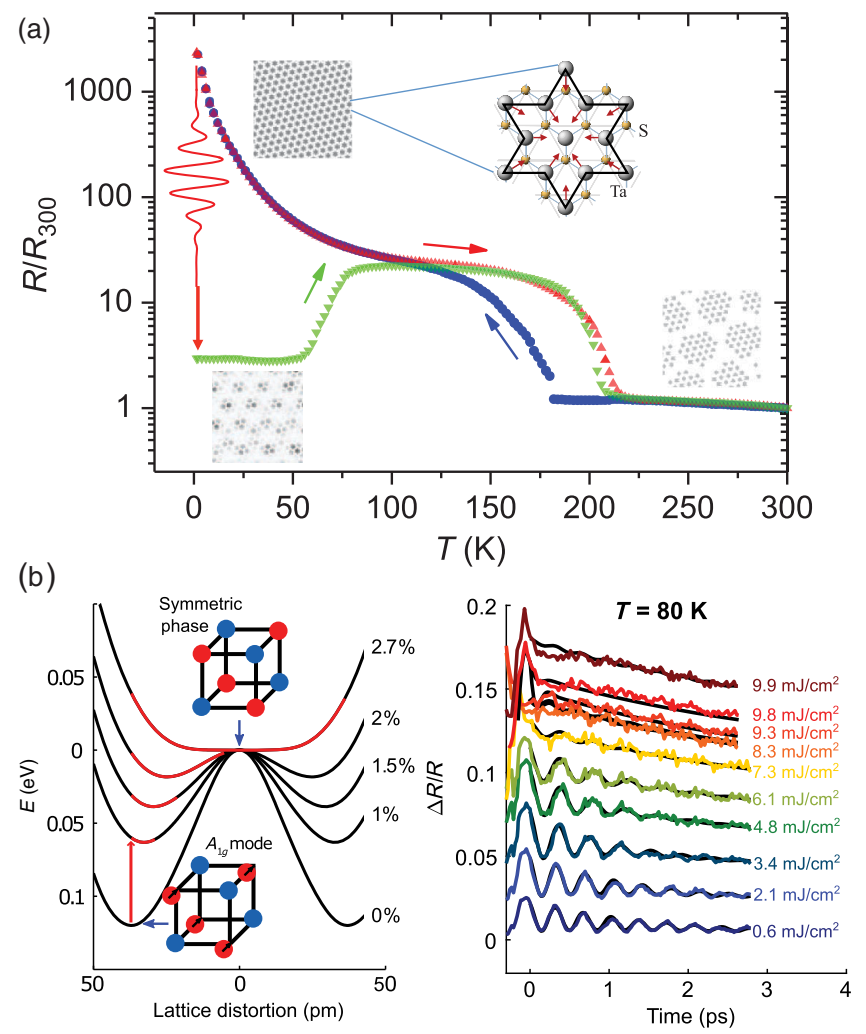

FIG. 4. Ultrafast switching. (a) At equilibrium $1 T-\mathrm{TaS}_{2}$ goes through successive charge-density wave (CDW) transitions as a function of temperature, resulting in an insulating low-temperature state that is characterized by strong correlations (blue circles, red up triangles). Upon photoexcitation with a single $35 \mathrm{fs}$ optical pulse, a long-lived metallic state emerges (green down triangles). Adapted from Vaskivskyi et al., 2015. (b) Photoexcitation of electrons in bismuth changes the local nuclear potential energy. For excitation densities above $2 \%$ the amplitude of the initiated $A_{1 g}$ mode drives a transition to a higher-symmetry phase. The $A_{1 g}$ phonon mode, characteristically for the low-symmetry phase, gets damped as the new phase is reached. Adapted from Teitelbaum et al., 2018.

2014; Zhang et al., 2016; Teitelbaum et al., 2019) or electric field (Asamitsu et al., 1997) pulses, and (iii) the emergence of a long-lived state with superconductinglike properties at higher temperatures than at equilibrium upon light-induced suppression of the charge (stripe) order in the 1/8-doped cuprates (Fausti et al., 2011; Nicoletti et al., 2014; Cremin et al., 2019) and in $\mathrm{K}_{3} \mathrm{C}_{60}$ (Mitrano et al., 2016; Cantaluppi et al., 2018; Budden et al., 2021).

These works have paved the way for moving the field toward developing new flexible, fast, and reversible switching strategies, for example, unimpeded by the healing of topological defects (Mihailovic, 2019; Mraz et al., 2021). Here the field of multiferroic materials (in which magnetism is controlled by dc electric fields) served as inspiration (Spaldin, 2020) for ultrafast experiments that reported on the engineering of ferroelectric states (Kubacka et al., 2014; Stoica et al., 2019). Ultrafast switching into a metastable ferroelectric phase has been observed after coherently driving the quantum paraelectric phase of $\mathrm{SrTiO}_{3}$ with strong terahertz fields 
(Li et al., 2019; Nova et al., 2019). This approach has also been used to demonstrate the ultrafast reversibility of the ferroelectric polarization in both directions, which is essential for future technological applications (Qi et al., 2009; Mankowsky et al., 2017). Additionally, the prospect of utilizing laser pulses to switch the topological state of a material was recently demonstrated in terahertz-driven $\mathrm{WTe}_{2}$ (Sie et al., 2019).

Finally, we discuss the key ideas behind symmetry-guided switching (Tsuji and Aoki, 2015; Chou, Liao, and Foster, 2017; Dehghani and Mitra, 2017; Sentef et al., 2017; Dasari and Eckstein, 2019; Kennes, Claassen et al., 2019; Ono and Ishihara, 2019; Dehghani, Hafezi, and Ghaemi, 2021) on the specific example of chiral superconductors (Claassen et al., 2019), which constitutes a theoretically proposed but not yet experimentally verified ultrafast switching paradigm. Going back to Fig. 2(b), this example corresponds to a free energy landscape with two minima $A$ and $B$ that represent the left- and right-handed chiral ground states of a topological superconductor. It was shown that a combination of linearly polarized laser pulses followed by circularly polarized laser pulses can nudge the system from the right- to the left-handed chiral ground state. The system can immediately be switched back equally quickly by applying another sequence of pulses and simply changing the chirality of the circularly polarized laser pulse. The key ingredient besides laser polarization is the duration of the two pulses: the circular pulse has to be applied when the system has not yet recovered from the linear pulse and remains in a superposition of the two chiral states. Recently, it was theoretically shown how the same mechanism, but with spatially localized laser spots, could be used to write, move, or erase chiral domains in real space (Yu et al., 2021).

\section{Out-of-equilibrium critical behavior}

Ultrafast photoexcitation can induce an instantaneous change of the free energy landscape by suppressing the relevant order parameter [Fig. 2(c)]. This phenomenology challenges our understanding of symmetry-breaking phase transitions. At equilibrium, the macroscopic time-dependent GL theory assumes an order parameter that varies sufficiently slowly in time. However, when the order parameter is suppressed on system-intrinsic timescales, it is unclear whether equilibrium concepts (universality classes, scaling) remain applicable and how the order-parameter fluctuations evolve after light excitation. Here we discuss out-of-equilibrium symmetry-breaking phase transitions both as a pathway toward accessing nonthermal effects and as a platform to study fundamental questions about macroscopic properties associated with order-parameter dynamics. The seminal works by Hohenberg and Halperin (1977), Polkovnikov et al. (2011), and Täuber (2017) offer comprehensive reviews.

An example of universal critical behavior in out-ofequilibrium phase transitions is the slowing down of the recovery dynamics. As occurs at thermal equilibrium, in the vicinity of a second-order transition spatial fluctuations of the order parameter diverge, and the exponential rate with which the ordered phase is reinstated vanishes following a

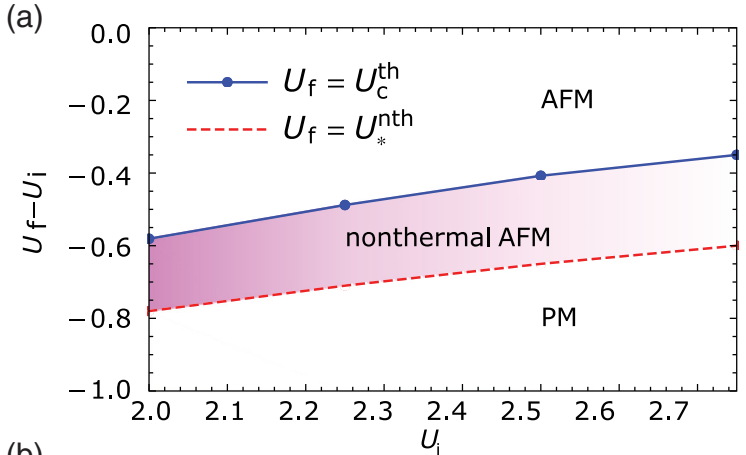

(b)

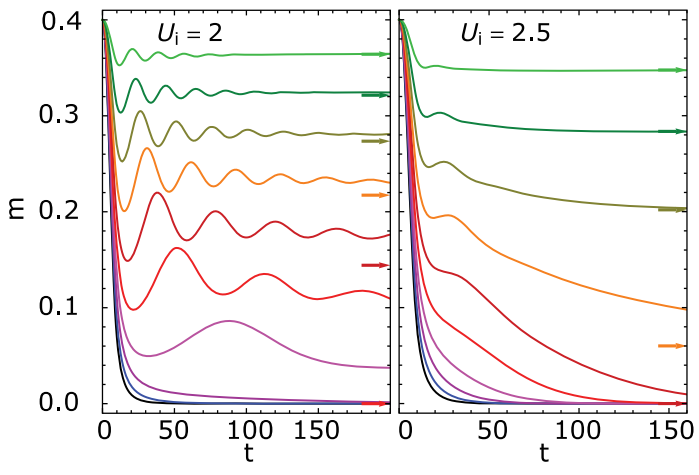

FIG. 5. Nonthermal critical behavior. (a) Nonequilibrium phase diagram as a function of $U_{\mathrm{i}}$ and size of the quench $U_{\mathrm{f}}-U_{\mathrm{i}}$. A splitting of the critical point into thermal and nonthermal antiferromagnetic (AFM) critical points is observed. (b) Time evolution of the magnetization for a quench of the electronelectron interaction $U_{\mathrm{i}} \rightarrow U_{\mathrm{f}}$ in the antiferromagnetic phase of the Hubbard model. Each color represents a different value of $U_{\mathrm{f}}$ (left panel: $U_{\mathrm{f}}=1.0,1.1, \ldots, 1.9$ and right panel: $U_{\mathrm{f}}=$ $1.5,1.6, \ldots, 2.4$ from bottom to top). Adapted from Tsuji, Eckstein, and Werner, 2013.

power law. This has been seen in the electronic recovery of charge order in perovskite manganites (Beaud et al., 2014) and $\mathrm{La}_{1 / 3} \mathrm{Sr}_{2 / 3} \mathrm{FeO}_{3}$ (Zhu et al., 2018), in the suppression dynamics of the charge-density wave in $\mathrm{LaTe}_{3}$ (Zong et al., 2019a), and in the spin and orbital dynamics in $\mathrm{YVO}_{3}$ and $\mathrm{GdVO}_{3}$ (Yusupov, Mihailovic et al., 2010) and $\mathrm{LaVO}_{3}$ (Lovinger et al., 2020). Divergent slow dynamics also characterizes the ultrafast suppression of the nematic electronic order in FeSe (Shimojima et al., 2019) and in iron pnictides (Patz et al., 2014). This seemingly universal behavior in out-of-equilibrium second-order phase transitions has been discussed in the time-dependent GL framework (Dolgirev et al., 2020), with stochastic Langevin equations describing the relevant subsystems (Sieberer, Buchhold, and Diehl, 2016), and with nonequilibrium Green's functions (Schüler, Murakami, and Werner, 2018).

Additionally, ultrafast phase transitions provide the possibility of studying the effects of nonadiabatic suppression. Near the phase transition the ordered and disordered phases are quasidegenerate in energy. This allows the fluctuations of the order parameter to be arbitrarily slow. Within the KibbleZurek mechanism (Kibble, 1976; Zurek, 1996), when the order-parameter suppression happens on timescales faster than those of the fluctuations, coherence between order-parameter domains is lost and the critical behavior is replaced by 
recovery dynamics dominated by the healing of topological defects. This behavior is universal (Bray, 1994) and has been observed in the long-time recovery dynamics after suppression of the striped phase in cuprates (Mitrano et al., 2019) and of the charge order in $\mathrm{LaTe}_{3}$ (Zong et al., 2019b), $\mathrm{LaTe}_{3}$ (Yusupov, Mertelj et al., 2010; Mertelj et al., 2013), and $1 T-\mathrm{TaS}_{2}$ (Vogelgesang et al., 2018). A different nonthermal scenario has been shown to emerge when two strongly coupled order parameters are quenched by a laser: In the striped nickelate $\mathrm{La}_{1.75} \mathrm{Sr}_{0.25} \mathrm{NiO}_{4}$ an absence of topological defects was reported in conjunction with slow phase fluctuation recovery (Lee et al., 2012; Chuang et al., 2013) and explained using a time-dependent GL theory (Kung et al., 2013).

We finally discuss predictions of nonthermal behavior in strongly correlated systems when they are driven through a phase transition (Chiocchetta et al., 2017). For example, quenching of the electron-electron repulsion $U$ in the antiferromagnetic phase of the Hubbard model results in a suppression of the magnetization. Depending on the size of the quench it was shown that a nonzero order parameter and coherent amplitude mode oscillations survive even for quenched values of $U$ at which the system would be in the disordered phase at thermal equilibrium (Werner, Tsuji, and Eckstein, 2012; Sandri and Fabrizio, 2013; Tsuji, Eckstein, and Werner, 2013; Balzer et al., 2015; Werner and Murakami, 2020). This gives rise to the prototypical out-of-equilibrium phase diagram shown in Fig. 5, where the suppression of the amplitude mode is associated with a secondary nonthermal critical point at $U_{\mathrm{f}}=U_{*}^{\text {nth }}$. Note that such a transition is not expected within the GL picture, where at the critical point the amplitude mode oscillations vanish as the curvature of the free energy potential is suppressed, and consequently the restoring force disappears (Hohenberg and Halperin, 1977).

\section{Nonlinearities and dynamical couplings}

We now discuss cases where nonlinear regimes are reached through intense excitation and showcase two examples: (i) dynamical effective interactions due to modified electronic screening, in which the nonlinearity stems from electronelectron interactions, and (ii) strongly driven crystal lattices, in which the nonlinearity is due to phonon-phonon interactions or other phonon-related nonlinearities. We note that this classification is mainly for pedagogical reasons. In reality, in both cases the effective Hamiltonian is transiently modified, and it is not always straightforward to reveal the microscopic origin of this modification. The first direct experimental evidence of transiently changed electron-electron interactions was interpreted in the context of (ii) (Kaiser et al., 2014a; Singla et al., 2015).

\section{Dynamical Hubbard $U$ interaction}

The dynamical regime after ultrafast photoexcitation opens a pathway for changing the effective interactions in correlated materials. The paradigmatic example for such an effective interaction is the Hubbard $U$, which parametrizes the screened local part of the Coulomb integrals of strongly localized (typically $d$ or $f$ ) orbitals. Examples of applications of the Hubbard model are transition-metal oxides (Imada, Fujimori, (a)
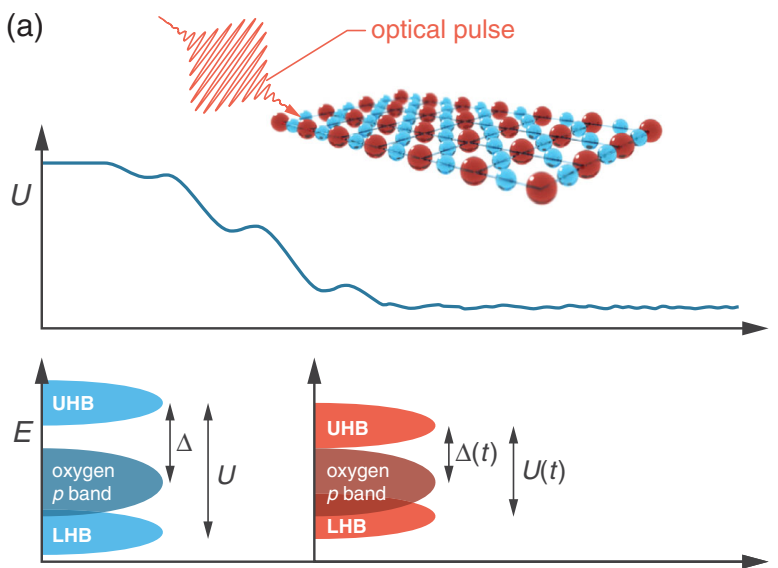

(b)
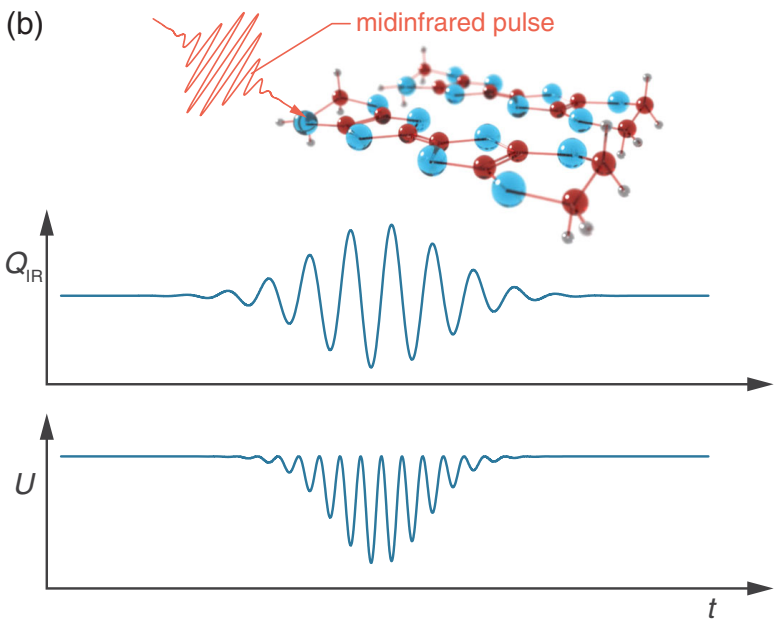

FIG. 6. Dynamically screened Hubbard $U$ interaction in laserdriven quantum materials. (a) Screening is typically enhanced when a material is excited by an optical laser pulse, which creates electronic excitations, resulting in a dynamically reduced timedependent $U$ (Tancogne-Dejean, Sentef, and Rubio, 2018; Golež, Eckstein, and Werner, 2019). In charge-transfer insulators this results in a narrowing of the Mott-Hubbard gap between the upper and lower Hubbard bands (UHB and LHB, respectively), as well as the charge-transfer gap $\Delta$ (Tancogne-Dejean, Sentef, and Rubio, 2020). (b) For a mid-IR laser excitation that is resonant with an IR-active phonon mode, the dynamical $U$ can parametrically depend on the phonon-mode coordinate $Q_{\mathrm{IR}}$ via a nonlinear electron-phonon interaction $\left(Q_{\mathrm{IR}}^{2} U\right)$. Whether $U$ increases or decreases on average depends on the sign of this nonlinearity.

and Tokura, 1998). There localized transition-metal ions are embedded between more delocalized ligand orbitals, which often are energetically separated from the $d$ orbitals. In such cases a downfolding procedure can be applied (Miyake, Aryasetiawan, and Imada, 2009) in which the low-energy effective model contains a Hubbard $U$ that depends on the screening from electrons in delocalized environmental orbitals. The key idea of a dynamical Hubbard $U$ interaction is that the electronic excitations created after interaction with an ultrafast laser pulse can modify the screening environment, effectively changing $U$ within femtosecond timescales [Fig. 6(a)]. 
To explain the underlying mechanism, we discuss here the transient reduction of $U$ upon photoexcitation in the correlated antiferromagnetic charge-transfer insulator $\mathrm{NiO}$ predicted within time-dependent density functional theory plus $U$ $($ TDDFT $+U$ ) (Tancogne-Dejean, Sentef, and Rubio, 2018). The underlying physics can be understood as follows: The electronic structure of $\mathrm{NiO}$ is such that the localized correlated subspace of mainly $d$-orbital character and the rest of the system of mainly $p$-orbital character can be considered independently. Photoexcitation of electrons from the localized subspace into delocalized states results in additional screening. This naturally leads to a decrease of the effective $U$. It is instructive to think of the excitation as an induced timedependent polarization of the localized electrons affecting the dielectric properties of the delocalized electrons, which is expected for strong driving fields even for frequencies away from optical transitions. Similar results were obtained by Golež, Eckstein, and Werner (2019) and Gillmeister et al. (2020) through different methods.

Experimental evidence for a dynamically modified Hubbard $U$ interaction by photoexcitation of electron-hole pairs was reported in the transition-metal dichalcogenide $\mathrm{MoTe}_{2}$ (Beaulieu et al., 2021). In this material, the uncorrelated band structure has two electronlike bands crossing the Fermi level $E_{F}$ at the edge of the Brillouin zone. However, this is not observed in photoemission. Instead, as explained by DFT $+U$, the Hubbard $U$ pushes the respective states above $E_{F}$. This effect can be partially undone on ultrafast timescales, as demonstrated by tr-ARPES. At $\sim 200 \mathrm{fs}$ after photoexcitation, an ultrafast Lifshitz transition was observed at which the Fermi surface topology changed because the electronlike bands were pushed below $E_{F}$, triggered by the dynamical reduction of $U$.

A different route toward dynamical modulation of the Hubbard $U$ interaction is the coherent driving of the crystal lattice. Dynamical $U$ through phonon driving was first demonstrated in a prototypical one-dimensional Mottinsulating charge-transfer salt (Kaiser et al., 2014a; Singla et al., 2015) and more recently reported as a microscopic mechanism for possible photomolecular superconductivity far above equilibrium $T_{\mathrm{c}}$ in two-dimensional molecular crystals (Buzzi et al., 2020; Tindall et al., 2020). The phononic route toward dynamical interactions is particularly suited for molecular crystals, where the effective local sites of the Hubbard model comprise entire molecules or parts thereof, which can be vibrationally excited and the on-site effective interaction thus changed [Fig. 6(b)] through the deformation of the intramolecular wave function during the vibration. The key advantage of resonant phonon excitation in the mid-IR frequency range compared to photodoping by optical pulses lies in the spectral selectivity of the excitation pathway. This leads us to the discussion of vibrational control of emergent quantum phenomena in Sec. II.D.2.

\section{Nonlinear phononics}

We now focus on resonant excitation of the crystal lattice with terahertz and mid-IR laser pulses. The notion of modeselective control of the crystal lattice in quantum materials was named "nonlinear phononics" and relies on the symmetries of the crystal and the phonon modes (Först, Manzoni et al., 2011; Subedi, Cavalleri, and Georges, 2014). There are three main differences from experiments where a pump interacts directly with the electronic degrees of freedom. (i) The energy from the pump is transferred into an initially coherent motion of an IR-active phonon mode, which then further interacts with other phonon modes, as well as, electrons, and other collective degrees of freedom in the system. (ii) The targeted excitation of a particular phonon mode opens additional pathways for mode-selective control that are not accessible in traditional driving schemes. (iii) Salient effects can already happen during the pump pulse and can often be understood as parametric excitation of degrees of freedom that are coupled to the driven mode.

We consider a crystal with an inversion center in which parity is a good quantum number and phonon modes can be grouped into even (Raman active) and odd (IR active). Lowest-order anharmonicity leads to a nonlinear coupling of the form $g_{\text {cubic }} Q_{\mathrm{IR}}^{2} Q_{\mathrm{R}}$ between an IR and a $\operatorname{Raman}(\mathrm{R})$ mode, with a coupling strength $g_{\text {cubic }}$ that is determined by the anharmonic potential. Coherent driving of the IR mode to large amplitudes [Fig. 7(a)] using strong mid-IR laser pulses couples to the Raman modes such that a unidirectional displacive force occurs that is proportional to the square of the IR-mode displacement [Fig. 7(b)]. The consequences of the respective transient crystal structures [Fig. 7(c)] on the electronic subsystem have been shown to potentially change the phases of quantum materials on ultrafast timescales (Mankowsky, Först, and Cavalleri, 2016). As an example of fingerprints of a transiently modified lattice structure in pump-probe experiments, we show transient Bragg peak intensities [Fig. 7(d)] from femtosecond x-ray diffraction, supported by $a b$ initio density functional theory calculations of the $\mathrm{Cu}-\mathrm{O}$ distance [Fig. 7(e)], in the $\mathrm{YBa}_{2} \mathrm{Cu}_{3} \mathrm{O}_{6.5}$ hightemperature superconductor (Mankowsky et al., 2014).

Among others, coherent control of the crystal lattice by driving to anharmonic regimes has been suggested as a mechanism to induce ultrafast phase transitions, such as lattice-controlled metal-insulator transitions (Rini et al., 2007), and to lead to states of matter without equilibrium counterparts, including possible light-induced superconductivity (Fausti et al., 2011; Hu et al., 2014; Kaiser et al., 2014b; Mankowsky et al., 2014). In turn, these groundbreaking experiments have stimulated considerable theoretical activity involving nonequilibrium superconductivity (Denny et al., 2015; Raines, Stanev, and Galitski, 2015; Kim et al., 2016; Knap et al., 2016; Komnik and Thorwart, 2016; Okamoto, Cavalleri, and Mathey, 2016; Sentef et al., 2016, 2017; Babadi et al., 2017; Coulthard et al., 2017; Kennes et al., 2017; Mazza and Georges, 2017; Murakami et al., 2017; Sentef, 2017; Dasari and Eckstein, 2018; Nava et al., 2018; Wang, Chen et al., 2018), as well as a debate on how we should understand and interpret optical superconductinglike signatures on transient timescales (Demsar, 2020; Zhang et al., 2020). Furthermore, coherent optical driving of the lattice has been suggested as a path toward switching applications (see Sec. II.B), as well as a means of mapping interatomic forces and potentials (von Hoegen et al., 2018; Kozina et al., 2019). Another developing subfield is phonomagnetism (Nova et al., 
(a)

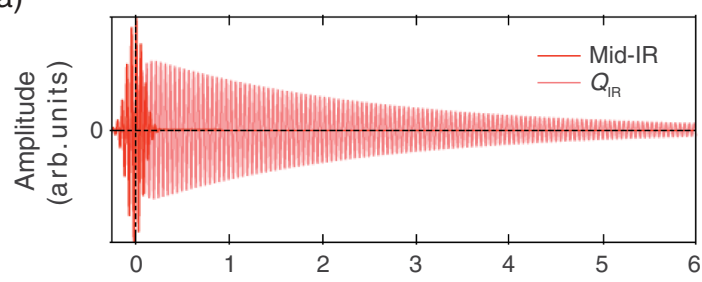

(b)

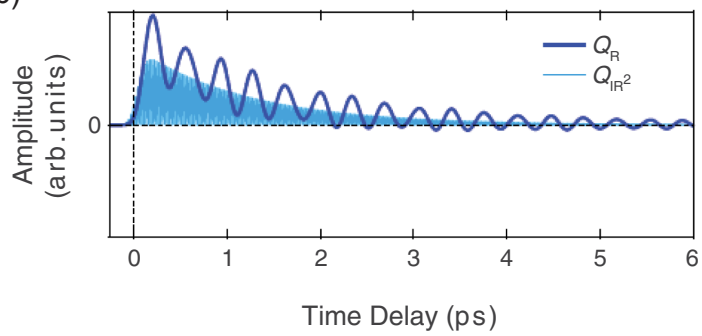

(c)

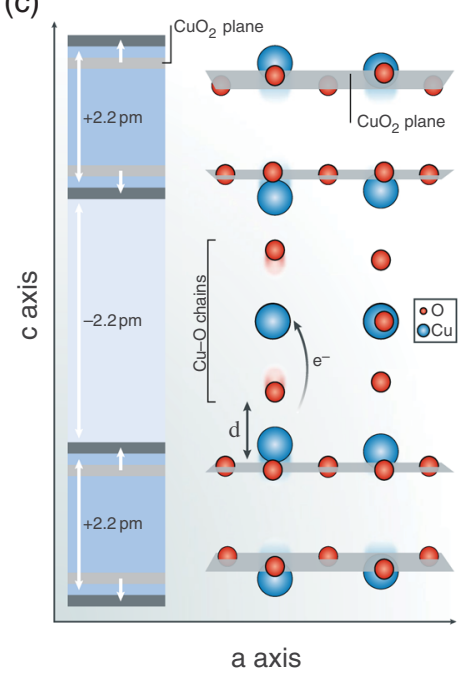

(d)

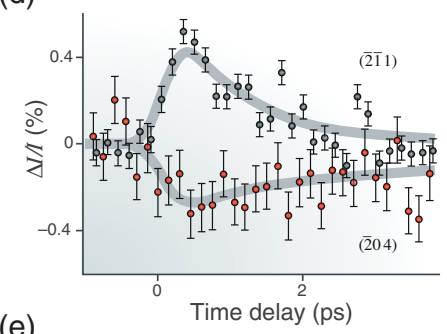

(e)

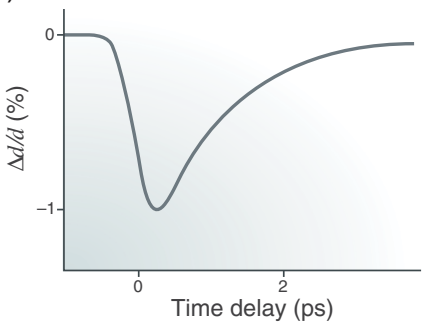

FIG. 7. Transient crystal structure from nonlinear phononics. (a) Oscillatory dynamics of an IR-active phonon-mode coordinate $Q_{\mathrm{IR}}$ is induced under resonant driving by a mid-IR laser pulse. (b) Owing to nonlinear phonon-phonon coupling to a Raman mode (coordinate $Q_{\mathrm{R}}$ ) of the form $Q_{\mathrm{IR}}^{2} Q_{\mathrm{R}}$, the potential energy of the Raman mode is transiently shifted to a new minimum following the squared amplitude $Q_{\mathrm{IR}}^{2}$. (a),(b) Adapted from Mankowsky et al., 2015. Accordingly, the Raman mode is displaced toward its transient potential minimum, which remains as long as the dynamics of the IR-active mode is coherent. (c) For $\mathrm{YBa}_{2} \mathrm{Cu}_{3} \mathrm{O}_{6.5}$, the motion of the optically excited coherent $B_{1 u}$ mode leads to a transiently modified crystal structure that was measured using time-resolved x-ray diffraction (Mankowsky et al., 2014) and is compatible with light-induced superconducting behavior. (d) Time-resolved relative change of intensity $\Delta I / I$ of two representative Bragg peaks. Solid curves show the ab initio calculated structure based on nonlinear phonon-mode coupling. (e) Transient change in the $\mathrm{Cu}-\mathrm{O}$ distance $d$ obtained from the calculation. (c)-(e) Adapted from Buzzi et al., 2018.

2017; Disa et al., 2020; Juraschek, Narang, and Spaldin, 2020; Afanasiev et al., 2021; Giorgianni et al., 2021; Stupakiewicz et al., 2021), in which magnetic properties of materials are modified via coherent phonon driving.

To summarize, phononic control of quantum materials is a rapidly growing research field, conceptually located at the boundary between ultrafast dynamics after short laser excitation and dressed states of nonequilibrium matter, that we discuss in the following.

\section{DRESSED STATES OF NONEQUILIBRIUM MATTER}

Another class of nonequilibrium phenomena encompasses the effects that occur in solids during optical illumination. Examples include high-harmonic generation (Ghimire et al., 2011; Schubert et al., 2014; von Hoegen et al., 2018), subcycle electron and spin dynamics (Kampfrath et al., 2011; Schultze et al., 2013; Lucchini et al., 2016; Reimann et al., 2018), light-field-driven currents (Schiffrin et al., 2013; Higuchi et al., 2017), and the parametric amplification of plasma waves (Rajasekaran et al., 2016). Comprehensive reviews of many of these topics were given by Kampfrath, Tanaka, and Nelson (2013) and Ghimire and Reis (2019). Here we focus on observables described by the coherent dressing of a material's quantum states by the rapidly oscillating light field. The manner and degree to which the states are dressed are determined by the amplitude, frequency, and polarization of the electromagnetic drive. Such a coherent light-matter interaction thereby provides an external control knob for transiently tuning the electronic and magnetic interactions in solids, which can be used to engineer quantum states with no equilibrium counterpart.

The consequences of photon dressing have long been investigated in the context of atomic systems and have led to applications in atomic clocks, precision spectroscopy, and quantum information processing (Scully and Zubairy, 1997). At the root of many of these phenomena is the alternating current (ac) Stark effect, a process whereby the oscillating electromagnetic field effectively shifts or splits electronic energy levels via the electric dipole interaction (Autler and Townes, 1955). This fundamental dressing effect has also been observed in solid-state systems using the strong ac fields in ultrafast laser pulses (Fröhlich, Nöthe, and Reimann, 1985; Mücke et al., 2001; Rossi and Kuhn, 2002; Schäfer and Wegener, 2002; Haug and Jauho, 2008; Bonitz, 2016; Reutzel et al., 2020).

Quantum materials can be dressed by light in more complex ways than isolated atomic systems by exploiting the additional degrees of freedom that interact with the light field. While this enhanced complexity is an opportunity for realizing novel material responses, it comes at the cost of increased dissipation, which leads to decoherence due to electron-electron, electron-phonon, and other scattering mechanisms. Far stronger optical fields are thereby required to overcome these sources of decoherence in driven solid-state electronic systems (Sato et al., 2019; Nuske et al., 2020; Aeschlimann et al., 2021), which has historically made dressed states difficult to experimentally access.

Advances in the generation of strong-field laser pulses now make it possible to routinely access regimes where dressing 
(a) Equilibrium

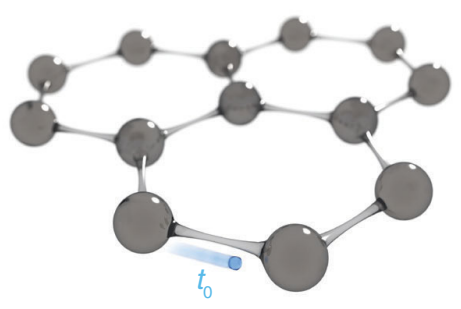

Optically dressed

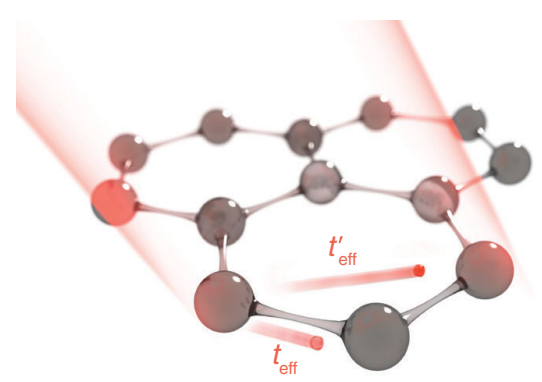

(b)
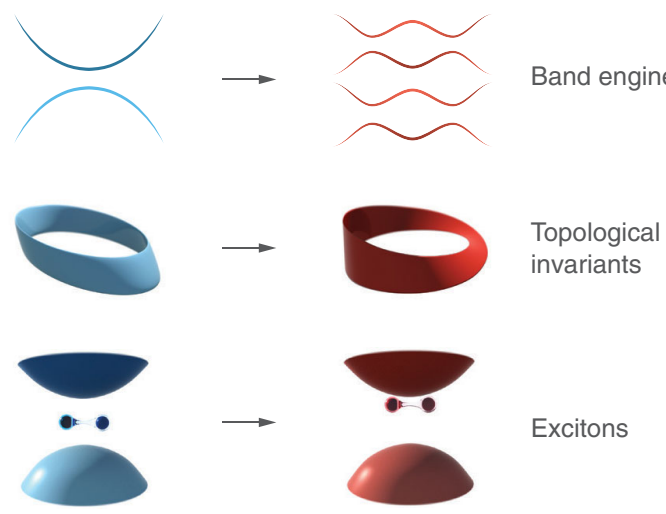

$\downarrow \uparrow \downarrow \uparrow \downarrow \downarrow \downarrow \rightarrow \uparrow \uparrow \uparrow \uparrow \uparrow \uparrow$ Magnetism

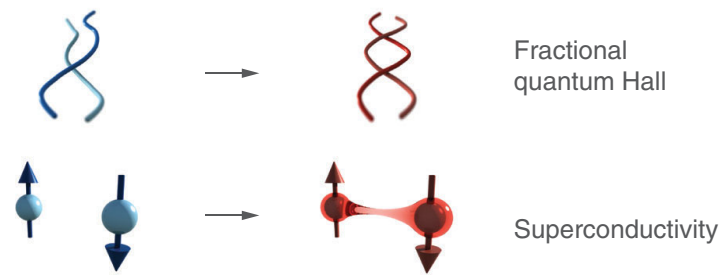

FIG. 8. Versatile functionalities enabled by Floquet engineering. (a) Periodic driving using optical fields can steer the macroscopic properties of quantum materials by dressing their microscopic degrees of freedom, such as electronic hopping parameters. (b) Examples include proposals and experimental realizations of new electronic band structures (Wang et al., 2013; Mahmood et al., 2016), the manipulation of band topologies (Oka and Aoki, 2009; Kitagawa et al., 2011; Lindner, Refael, and Galitski, 2011; McIver et al., 2020), and the observation of excitonic Stark shifts caused by the creation of strong synthetic magnetic fields (Kim et al., 2014; Sie et al., 2015). Additional proposals include the optical control of magnetic correlations (Mentink, Balzer, and Eckstein, 2015; Claassen et al., 2017; Kennes et al., 2018) that have been experimentally observed in quantum simulation settings (Görg et al., 2018), the creation of fractionized quasiparticles obeying non-Abelian statistics (Lee et al., 2018), and forms of photon-dressed superconductivity (Knap et al., 2016; Murakami et al., 2017; Kennes, Claassen et al., 2019; Tindall et al., 2020).

effects dominate the response of optically driven quantum materials, which are being investigated with the aid of a new generation of ultrafast experimental probes (Wang et al., 2013; McIver et al., 2020). Theoretical breakthroughs utilizing Floquet theory (Lindner, Refael, and Galitski, 2011; Oka and Kitamura, 2019; Rudner and Lindner, 2020) are simultaneously offering unprecedented guidance for experiments. This combined effort has led to remarkable discoveries and predictions (Fig. 8). We review some of these developments in the following.

\section{A. Theory introduction}

To introduce the concept of dressed states in solids, we begin with the intuitive example of a periodically driven atomic chain in the high-frequency limit. We then generalize to the case of arbitrary frequency, for which Floquet theory provides a flexible and powerful framework to describe dressing effects.

\section{Dynamical localization in a driven atomic chain}

Consider the following one-dimensional tight-binding model of noninteracting spinless fermions in second quantization:

$$
H=-\sum_{n} t_{0} c_{n}^{\dagger} c_{n+1}+\text { H.c. }=\sum_{k} \epsilon(k) c_{k}^{\dagger} c_{k}
$$

where $t_{0}$ is the hopping amplitude and $c_{n}^{(\dagger)}$ annihilates (creates) a fermion on site $n$. In momentum space, one obtains a dispersion relation $\epsilon(k)=-2 t_{0} \cos (k a)$, where $a$ is the lattice constant.

Next consider a coupling to a time-periodic longitudinal electric field $E(t)=E_{0} \sin (\omega t)$ with a field strength $E_{0}$ and frequency $\omega$, which can be included using the Peierls substitution $t_{0} \rightarrow t_{\mathrm{h}}(t)=t_{0} e^{i e a A(t) / \hbar}$, where $A(t)=E_{0} / \omega \cos (\omega t)$ is the vector potential, such that $E(t)=-\partial_{t} A(t)$. When the driving frequency is larger than the intrinsic energy and timescales in the system, the high-frequency oscillations cancel each other. Time averaging $t_{\mathrm{h}}(t)$, denoted by $\langle\cdots\rangle$, yields $t_{\text {eff }}=\left\langle t_{\mathrm{h}}(t)\right\rangle=t_{0} J_{0}(F)$, where $J_{0}$ is the 0th-order Bessel function. We introduced the dimensionless Floquet parameter

$$
F=\frac{a e E_{0}}{\hbar \omega}
$$

to parametrize the strength of the electromagnetic drive, with $e$ the elementary charge. Since $\left|J_{0}(x)\right|<1$, the resulting effective hopping amplitude $t_{\text {eff }}$ is always smaller than the 
(a)

Real time picture

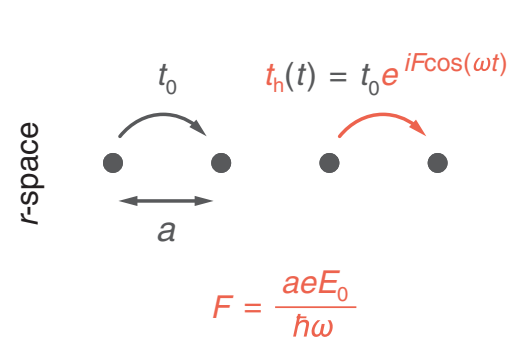

$k(t) \rightarrow k-\frac{e E_{0}}{\hbar \omega} \cos (\omega t)$

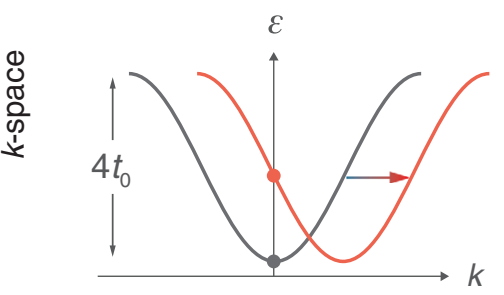

Effective picture

$t_{\text {eff }}=\left\langle t_{\mathrm{n}}(t)\right\rangle<t_{0}$

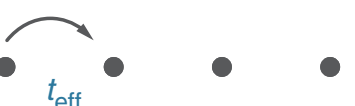

$t_{\text {eff }}$
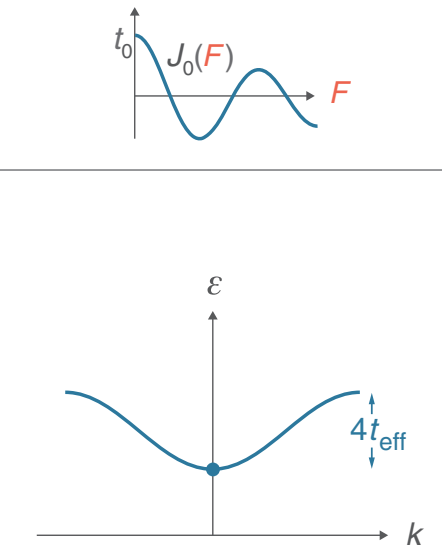

(b)

Floquet sidebands

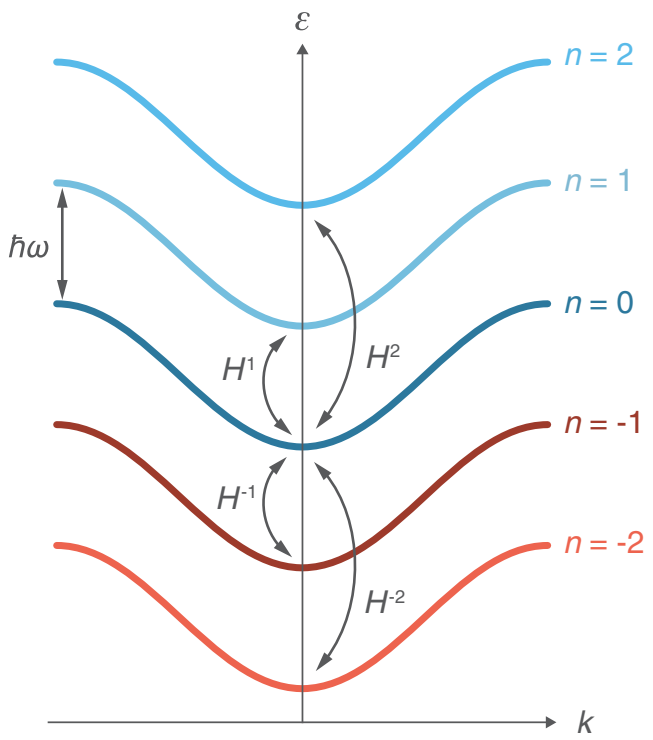

FIG. 9. Dynamical localization in an atomic chain and Floquet sidebands. (a) Illustration of dynamical localization in an atomic chain visualized in real (upper row) and momentum space (lower row). The two columns represent the real-time picture (left panel) as well as the effective, time-averaged picture (right panel) of the periodically driven system. When the equilibrium band dispersion (black, lower left panel) is driven back and forth in momentum space by the light field (red), the total bandwidth is reduced in the effective dressed picture (blue, lower right panel). This corresponds to a decrease in the effective hopping amplitude in real space, described by the Bessel function $t_{\text {eff }}=t_{0} J_{0}(F)$, shown in the upper right panel. (b) Visual representation of Floquet replicas according to Eq. (6). At high frequency $(\omega \rightarrow \infty)$ the effective Hamiltonian is given by the time average $H^{0}$. At finite frequency Floquet sidebands at energy distance $\omega$ become accessible via the higher moments $H^{1}, H^{2}, \ldots$ of the Fourier series of the time-dependent Hamiltonian $H(t)$.

equilibrium value $t_{0}$, meaning that on average (i.e., in the effective or dressed picture) the electrons in the driven chain are more localized than those in the undriven chain. This realspace description of dynamical localization is depicted in Fig. 9(a).

Equivalently, in momentum space the effect of dynamical localization is captured by the minimal coupling $k \rightarrow k-e A(t) / \hbar$ in the band dispersion. The effect of the electric field is to periodically shake the dispersion to the left and right [bottom row of Fig. 9(a)]. When considering the minimum of the equilibrium dispersion relation at $k=0$, one finds that this shaking on average shifts the bottom of the band dispersion to higher energy in the effective picture. Analogously, the dispersion maxima at the zone boundaries are lowered in energy upon averaging. The net effect in the effective, or dressed, picture is a reduction in bandwidth compared to the equilibrium dispersion relation. This example demonstrates how periodic driving provides a tuning knob for material properties via the renormalization of electronic hopping (Dunlap and Kenkre, 1986; Bucksbaum et al., 1990; Mentink, Balzer, and Eckstein, 2015; Claassen et al., 2017; Kennes et al., 2018; Kennes, Claassen et al., 2019).

\section{Floquet primer}

Floquet theory provides a powerful basis to treat a periodically driven quantum system beyond high- or low-frequency expansions. Such a system is governed by the following time-dependent Schrödinger equation:

$$
i \hbar \frac{d}{d t}|\Psi(t)\rangle=H(t)|\Psi(t)\rangle
$$

with a time-periodic Hamiltonian $H(t+T)=H(t)$ with period $T$. Floquet (1883) showed that the solution to Eq. (3) can be written as

$$
\left|\Psi_{l}(t)\right\rangle=e^{-i \epsilon_{l} t / \hbar}\left|\Phi_{l}(t)\right\rangle
$$

where the $T$-periodic wave function $\left|\Phi_{l}(t)\right\rangle=\left|\Phi_{l}(t+T)\right\rangle$ and the quasienergies $\epsilon_{l}$ are determined by the algebraic equation

$$
\left(\epsilon_{l}+n \hbar \omega\right)\left|\Phi_{l}^{n}\right\rangle=\sum_{n^{\prime}} H^{n-n^{\prime}}\left|\Phi_{l}^{n^{\prime}}\right\rangle
$$

with frequency $\omega=2 \pi / T$ and Fourier decompositions $H^{n}=$ $(1 / T) \int_{0}^{T} d t e^{i n \omega t} H(t)$ and $\left|\Phi_{l}^{n}\right\rangle=(1 / T) \int_{0}^{T} d t e^{i n \omega t}\left|\Phi_{l}(t)\right\rangle$. This maps a periodic differential equation to an algebraic (quasiequilibrium) problem. The time-dependent problem is transformed into an effective static Hamiltonian by considering $\underline{\Phi}_{l}=\left(\ldots,\left|\Phi_{l}^{-1}\right\rangle\left|\Phi_{l}^{0}\right\rangle\left|\Phi_{l}^{1}\right\rangle, \ldots\right)^{T}$ and $\mathcal{H} \underline{\Phi}_{l}=\epsilon_{l} \underline{\Phi}_{l}$, such that 
(a)

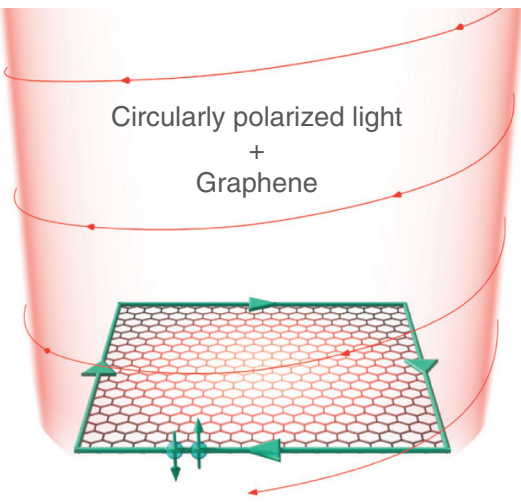

Dressed chiral edge states (b)
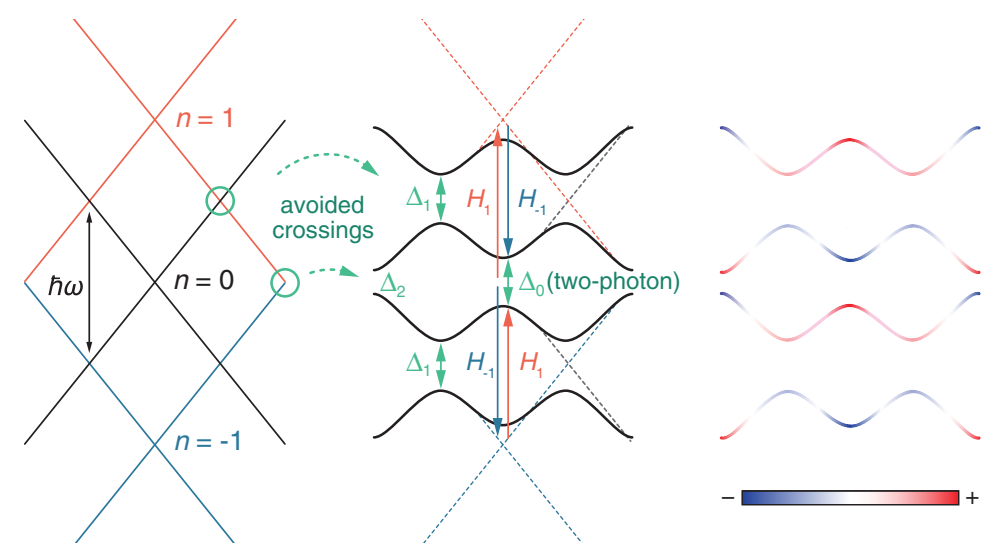

Before hybridization
After hybridization Berry curvature distribution (c)

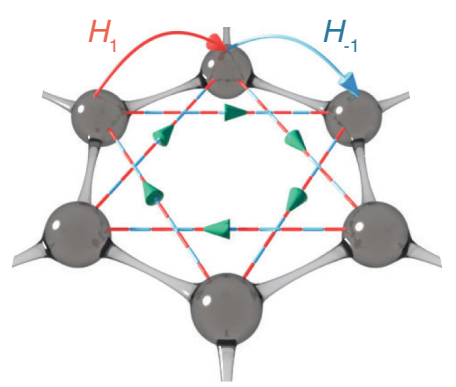

Two-photon absorption/emission (d)

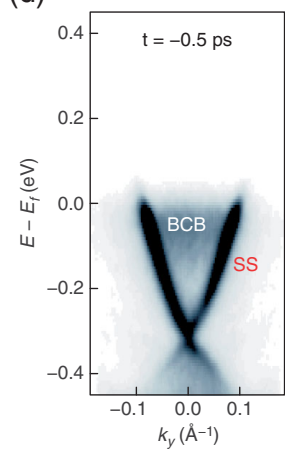

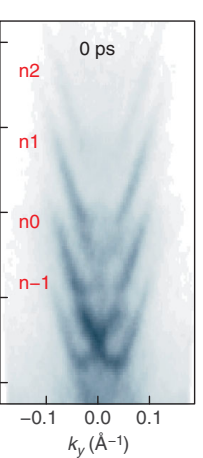

(e)

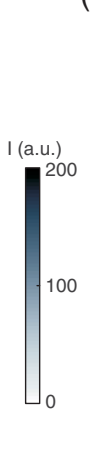

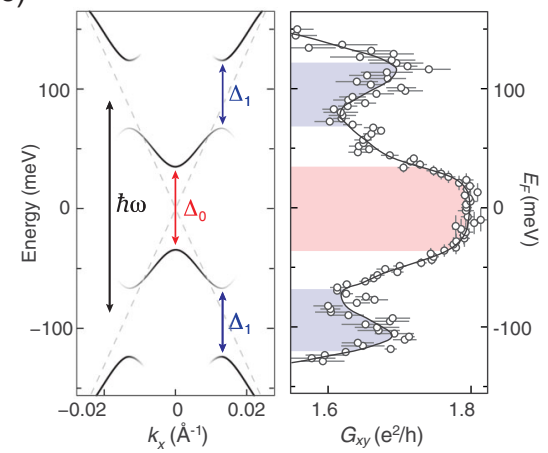

FIG. 10. Topological Floquet engineering in Dirac systems. (a) A coherent interaction with circularly polarized light was predicted to induce a photon-dressed topological band structure in graphene that is characterized by protected chiral edge states (Oka and Aoki, 2009; Kitagawa et al., 2011). (b) When the Floquet sidebands form, they hybridize at their crossing points, opening band gaps $\left(\Delta_{1}\right.$, $\left.\Delta_{2}\right)$. A gap also opens at the Dirac point $\left(\Delta_{0}\right)$ due to a two-photon absorption-emission process that breaks time-reversal symmetry. The resulting Floquet-Bloch bands are fully gapped and possess a nonzero total Berry curvature and Chern number. (c) Real-space schematic of the two-photon absorption-emission hopping process that induces a gap at the Dirac point in graphene. (d) tr- $\mathrm{ARPES}$ data of $\mathrm{Bi}_{2} \mathrm{Se}_{3}$ detecting the formation of Floquet-Bloch bands from the Dirac surface states. From Mahmood et al., 2016. (e) Nonequilibrium anomalous Hall conductance of graphene driven by circularly polarized light as a function of the equilibrium Fermi level $\left(E_{F}\right)$. Red and blue shading corresponds to $E_{F}$ being gated to the regions where the gaps $\Delta_{0}$ and $\Delta_{1}$ were predicted to appear. From McIver $e t$ al., 2020.

$$
\mathcal{H}=\left(\begin{array}{ccccc}
\ddots & H^{-1} & H^{-2} & H^{-3} & H^{-4} \\
H^{1} & H^{0}-(-1) \hbar \omega & H^{-1} & H^{-2} & H^{-3} \\
H^{2} & H^{1} & H^{0}-0 \hbar \omega & H^{-1} & H^{-2} \\
H^{3} & H^{2} & H^{1} & H^{0}-1 \hbar \omega & H^{-1} \\
H^{4} & H^{3} & H^{2} & H^{1} & \ddots
\end{array}\right) .
$$

$\mathcal{H}$ consists of an infinite number of copies of the timeaveraged Hamiltonian $H^{0}$ energetically shifted by integer multiples of the driving frequency $\omega$. The higher harmonics $H^{1}, H^{2}, \ldots$ couple the nearest, next-nearest, etc., neighboring copies in this effective language. In the context of solids, where the undriven system is described by Bloch bands, these copies, or Floquet replicas, lead to Floquet-Bloch bands repeating periodically in momentum (Bloch) as well as energy (Floquet) space [Fig. 9(b)]. This insight results in the notion of sidebands emerging under periodic driving, which can be used to alter the physical properties of a system dramatically
(Oka and Aoki, 2009; Kennes, Müller et al., 2019; Rudner and Lindner, 2020) (Fig. 10). Although Floquet theory was introduced more than 100 years ago, its implementation within new theoretical approaches (Sec. A.2) is a subject of intense research. Green's functions, other diagrammatics, or effective single-particle pictures, such as those used in dynamical mean-field theory, functional renormalization group, and density functional theory, can easily exploit these ideas (Tsuji, Oka, and Aoki, 2008; De Giovannini et al., 2013; Aoki et al., 2014; Eissing, Meden, and Kennes, 2016). For tensor networks (Kennes et al., 2018; Sahoo, Schneider, and Eggert, 2019) and other variational techniques this is substantially complicated by the fact that Floquet theory requires the determination of the full spectrum.

Floquet engineering is challenging in quantum materials owing to the large optical fields $\left(10^{7}-10^{8} \mathrm{~V} / \mathrm{m}\right)$ and long wavelengths needed to obtain a sizable Floquet parameter [Eq. (2)]. This necessitates the use of intense ultrafast laser pulses and limits current experiments to the low-frequency regime. While Floquet theory inherently assumes continuous 
wave driving, experimental results obtained using laser pulses with multiple optical cycles have nevertheless been well described using this framework (Wang et al., 2013; Kim et al., 2014; Sie et al., 2015; Mahmood et al., 2016; McIver et al., 2020). We review some of these experimental results in the following.

\section{B. Dressed band structures: Floquet topological insulators}

Optically dressed quantum materials garnered considerable interest after they were proposed to be capable of hosting topologically nontrivial states that could be manipulated by adjusting the light field (Oka and Aoki, 2009; Kitagawa et al., 2011; Lindner, Refael, and Galitski, 2011). Such systems are often referred to as "Floquet topological insulators," and in some cases they are characterized by topological invariants that have no equilibrium counterpart (Kitagawa et al., 2010; Rudner et al., 2013).

The first proposal along these lines was put forward by Oka and Aoki (2009). Using Floquet theory they showed that an anomalous Hall effect could be induced in graphene by dressing its quantum states with circularly polarized light [Fig. 10(a)] (Oka and Kitamura, 2019). The physics can be summarized as follows: Floquet-Bloch bands form and hybridize at their crossing points, opening band gaps $\left[\Delta_{1}\right.$, $\Delta_{2}$ in Fig. 10(b)]. A second-order process also causes a gap to open at the Dirac point $\left(\Delta_{0}\right)$ because the interaction breaks time-reversal symmetry. The resulting fully gapped FloquetBloch bands have a nonzero Berry curvature distribution that causes charges to undergo an anomalous Hall effect. By reversing the helicity of the light, the polarity of the induced Berry curvature distribution, and hence the direction of the net anomalous Hall current, can be controlled.

In the high-frequency limit, where the Floquet sidebands do not overlap and thus there are no gaps due to band crossings, the Hamiltonian for the driven system is

$$
H_{\mathrm{eff}} \approx H_{0}+\frac{\left[H_{-1}, H_{1}\right]}{\hbar \omega},
$$

where the first term is the equilibrium Dirac Hamiltonian and the second term is responsible for the gap opening at the Dirac point. Specifically, for a two-dimensional Dirac cone with energy-momentum relation $\epsilon(\vec{k})=\hbar v_{F}|\vec{k}|$ with Fermi velocity $v_{F}$, one obtains $\Delta_{0}=2 \hbar^{-1} e^{2} v_{F}^{2} E_{0}^{2} / \omega^{3}=2 \hbar^{-1} e^{2} v_{F}^{2} A_{0}^{2} / \omega$, which scales quadratically with the peak electric field $E_{0}$ and is enhanced for small frequencies.

Kitagawa et al. (2011) interpreted Eq. (7) as the energy difference $\left(\Delta_{0}\right)$ between two processes, where an electron in the $n=0$ Dirac cone first absorbs (emits) a circularly polarized photon to enter the $n=1(n=-1)$ sector, then emits (absorbs) a photon of the same helicity to return to the $n=0$ sector [Fig. 10(b)]. In real space, this corresponds to a double hopping in the unit cell that has the net effect of introducing chiral intrasublattice tunneling elements to the effective Hamiltonian [Fig. 10(c)]. The effective Hamiltonian is identical to that proposed by Haldane (1988) for a topological Chern insulator in graphene, which was previously believed to be inaccessible via any realistic experimental setting. When only the lower Chern band is populated, a topological phase is expected to develop that hosts a quantized anomalous Hall effect carried by chiral edge states, which would be photon dressed in this case.

Floquet topological insulators have been studied in the high-frequency limit using a variety of quantum simulation platforms, most notably in photonic waveguides (Rechtsman et al., 2013), where the propagating edge mode was directly observed, and in driven optical lattices of ultracold fermions (Jotzu et al., 2014). Later theoretical works confirmed that topological states could also be created in the low-frequency limit [Fig. 10(b)], with the difference being that the multitude of Floquet-Bloch bands that form due to the additional gap openings can have Chern numbers $|C|>1$, i.e., multiple edge modes (Usaj et al., 2014; Dehghani, Oka, and Mitra, 2015; Sentef et al., 2015; Claassen et al., 2016; Mikami et al., 2016). An additional topological invariant named the winding number can also arise in the low-frequency limit (Kitagawa et al., 2010; Rudner et al., 2013), which is defined over time rather than momentum space, and thus realizable only in periodically driven systems. Observations of such "anomalous Floquet topological insulators" have also been reported in the quantum simulation community (Kitagawa et al., 2012; Maczewsky et al., 2017).

In a landmark paper, Wang et al. (2013) reported the first spectroscopic evidence of topological Floquet-Bloch bands in a solid within the Dirac surface states of the topological insulator $\mathrm{Bi}_{2} \mathrm{Se}_{3}$ using tr-ARPES [Fig. 10(d)]. The data reveal multiple dressed sidebands and the opening of band gaps similar to those illustrated in [Fig. 10(b)].

McIver et al. (2020) reported the first electrical transport results from topological Floquet-Bloch bands. Using an ultrafast transport device (bottom center panel of Fig. 1), they detected an anomalous Hall effect in graphene illuminated by a circularly polarized mid-IR pulse. At high laser pulse fluences they observed that the anomalous Hall conductance saturated of the order of 2 conductance quanta, the same value predicted in the high-frequency limit (Kitagawa et al., 2011). When the graphene Fermi level was tuned away from charge neutrality using an electrostatic gate [Fig. 10(e)], features were observed in the conductance spectrum that were closely aligned with the opening of the band gaps $\Delta_{0}$ and $\Delta_{1}$, as predicted by Floquet theory for their laser pulse parameters. Subsequent numerical studies confirmed that the observed photocurrents originated from the formation of topological Floquet-Bloch bands (Sato et al., 2019; Nuske et al., 2020). These results are an encouraging sign that Floquet-engineered topological edge states are a distinct possibility in optically driven Dirac materials.

\section{Toward Floquet many-body physics: Heating and interactions}

Experiments by Wang et al. (2013) and McIver et al. (2020) demonstrated that Floquet engineering in quantum materials is realistic despite the presence of strong dissipation, which leads to decoherence. However, the tr-ARPES data also reveal significant pump-induced carrier excitation, leading to a nonequilibrium dressed electron distribution in the FloquetBloch bands. Here distributional and dissipative effects already play an important role, even in the absence of electronic interactions (Dehghani, Oka, and Mitra, 2014; 
Iadecola, Neupert, and Chamon, 2015; Seetharam et al., 2015), and contribute to substantial deviations from quantization of the Hall conductivity and edge state transport in Floquet band topological insulators (Kundu and Seradjeh, 2013; Farrell and Pereg-Barnea, 2016). The roles of heating and thermalization hence can be expected to take on an outsized role as "Floquet engineering" is extended toward the control of interacting many-body states of matter. Therefore, materials and driving protocols need to be chosen carefully in order to minimize heating and carrier redistribution such that a nonequilibrium state proximal to the ground state of the effective Hamiltonian is realized.

Theoretically, there have been multiple proposals to address the challenge of heating. In general, closed periodically driven interacting systems continuously absorb energy and heat to a featureless infinite-temperature steady state by virtue of the eigenstate thermalization hypothesis for nonintegrable systems (D'Alessio and Rigol, 2014). However, tailored drive protocols can be chosen to suppress heating at short times and realize a potentially long-lived "prethermal" regime. Here a central ingredient is a separation of energy scales, and thus a separation of timescales in the driven system, which entails an ergodic obstruction to fast energy absorption. After an initial ramp-on period governed by fast switch-on processes, the intermediate time (period-averaged) dynamics can saturate to a prethermal plateau, governed by an effective Hamiltonian that captures a controlled Floquet modification, leading to observable consequences in correlation functions or spectroscopic probes of the system. This regime is bounded by a timescale that signifies the onset of heating toward a featureless high-temperature state (Fig. 11), which can in principle nevertheless retain certain correlations when constrained by exact non-Abelian symmetries (Tindall et al., 2019).

Most of our current understanding regards the limit of highfrequency driving (Mentink, Balzer, and Eckstein, 2015; Claassen et al., 2017; Weidinger and Knap, 2017; Haldar, Moessner, and Das, 2018; Peronaci, Schiro, and Parcollet, 2018; Vajna et al., 2018; Machado et al., 2019, 2020; Gulden et al., 2020), where the pump frequency exceeds local energy scales. In this limit rigorous results for slow heating were recently established (Abanin, De Roeck, and Huveneers, 2015; Kuwahara, Mori, and Saito, 2016; Mori, Kuwahara, and Saito, 2016; Abanin et al., 2017a, 2017b; Ho, Protopopov, and Abanin, 2018; Mori et al., 2018). These works provide only mathematical bounds for the onset of heating to featureless states at infinite temperature, which can be exceeded in specific settings. Many-body localized (MBL) systems constitute an important exception (D'Alessio and Polkovnikov, 2013; Lazarides, Das, and Moessner, 2014, 2015; Ponte et al., 2015). Here heating can be averted via a lack of ergodicity, stabilizing a tantalizing array of bona fide nonequilibrium phases, such as time-crystalline orders or Floquet topological phases (Zeng and Sheng, 2017; Rovny, Blum, and Barrett, 2018; Decker et al., 2020).

In practice, a high-frequency driving limit is absent from most real materials since higher-lying bands and collective excitations provide a multitude of possibilities for resonant absorption at higher frequencies. Similarly, while MBL is realizable in cold atomic systems (Singh et al., 2019), it is typically destabilized in solids due to energy dissipation to the (a) Tuning prethermal states

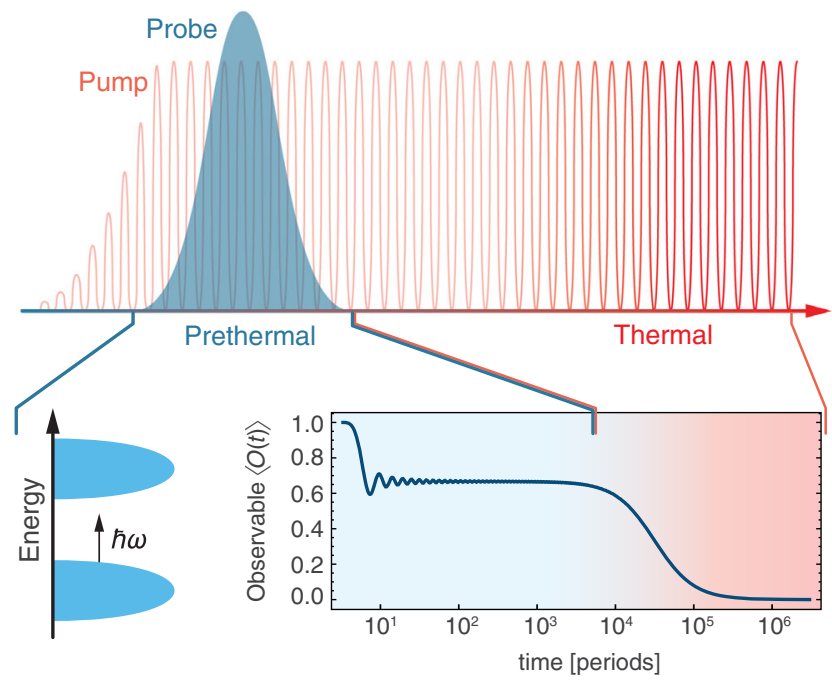

(b) Engineering correlated systems
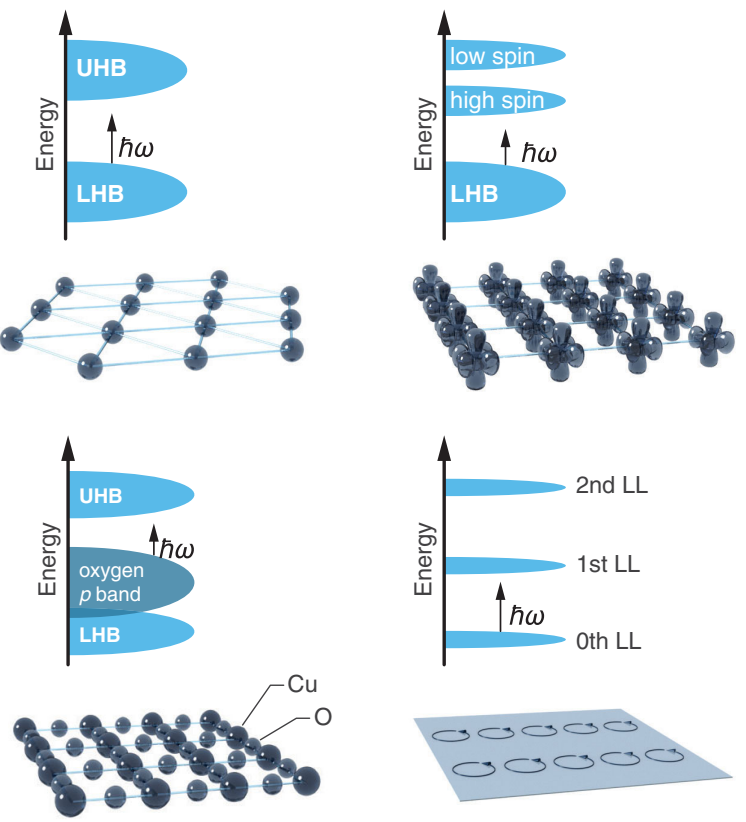

FIG. 11. Routes to avoid runaway heating. (a) Generic interacting systems heat up upon applying a continuous drive, which is detrimental to coherent control. However, when the drive frequency is tuned to a spectral gap in the system, this heating can in principle be pushed to exponentially large times. Control of prethermal states can then be achieved on intermediate to long timescales until thermalization sets in. (b) Examples of condensed matter systems for which such a strategy could be applied. The upper two and bottom left panels show Mott and charge-transfer insulators (LHB and UHB denote the lower and upper Hubbard bands, respectively). Another example involves quantum Hall insulators (lower right panel). Between the different Landau levels (LLs) energy gaps proportional to the externally applied magnetic field emerge. Frequency detuning to the sharp resonances between LLs could in principle allow for prethermal state Floquet control of the system.

lattice or other degrees of freedom. Nonetheless, sufficiently slow energy absorption, for instance, due to off-resonant driving or other ergodic obstructions to heating, can realize 

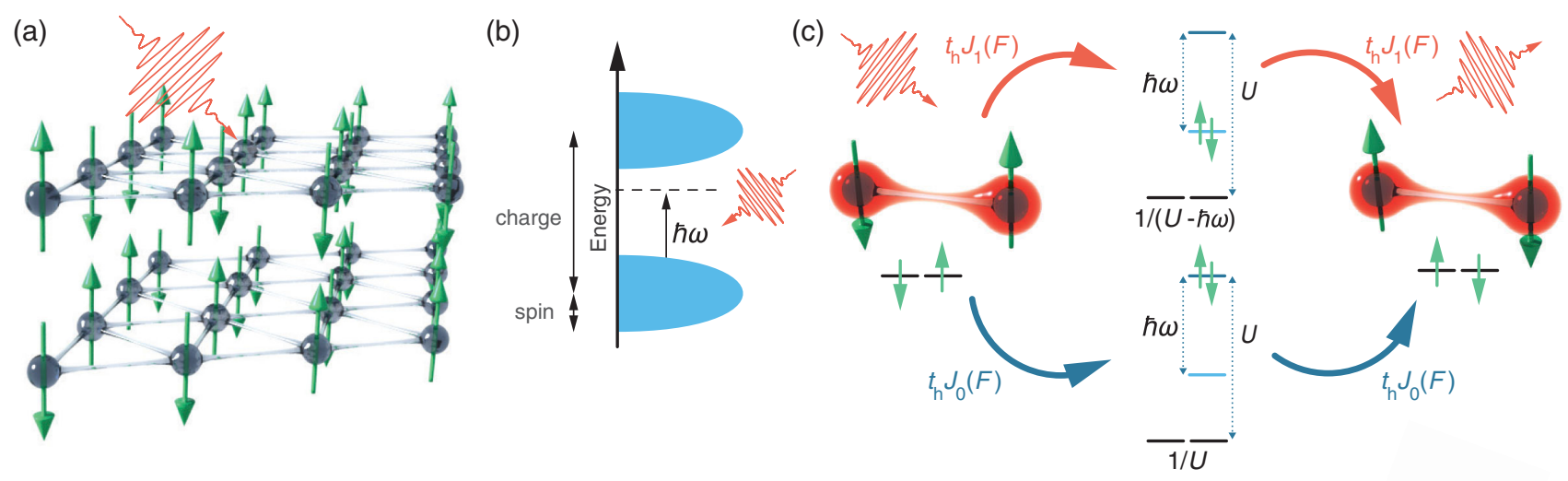

(d)

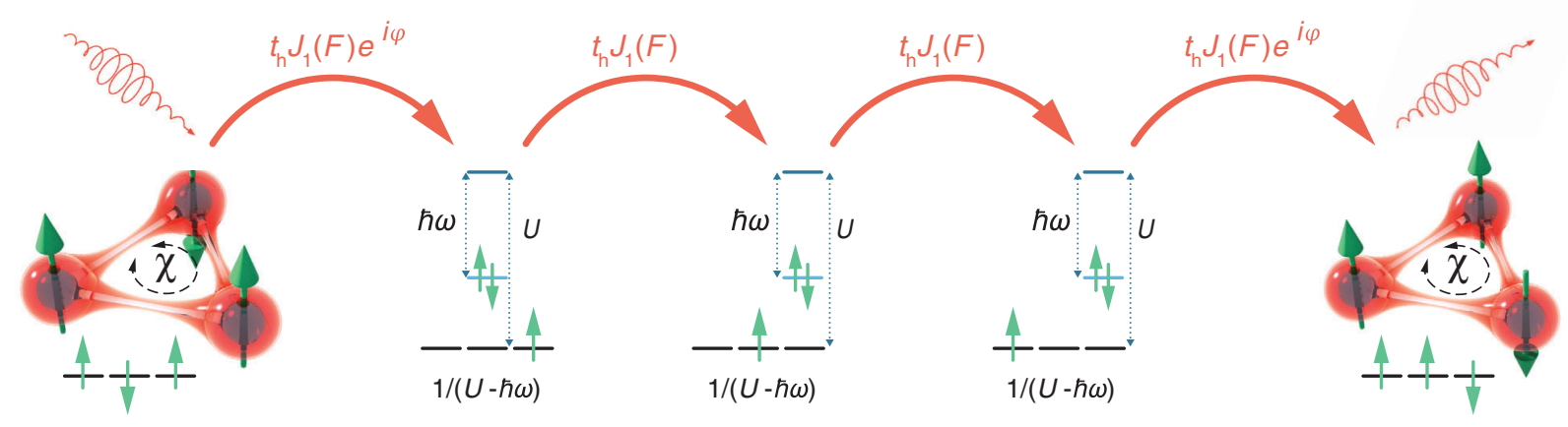

FIG. 12. Floquet engineering of magnetic interactions. (a) Schematic of manipulation of magnetic degrees of freedom via irradiation of quantum magnets with light. (b) In Mott insulators, pumping below the charge gap suppresses charge excitations on short timescales while renormalizing the magnetic interactions at low energies. (c) Irradiation with light can alter conventional spin-exchange interactions by reducing the energy cost of intermediate virtual states of electronic tunneling. (d) Circularly polarized light can induce new types of magnetic interactions, such as SU(2)-symmetric chiral three-spin-exchange interactions due to dynamical time-reversal symmetry breaking, which are absent at equilibrium.

"prethermal" dynamical regimes at short times (Fig. 11), which can harbor intriguing emergent phenomena (Haldar, Moessner, and Das, 2018; Rovny, Blum, and Barrett, 2018; Gulden et al., 2020; Luitz et al., 2020; Claassen, 2021). Therefore, a central problem is to identify materials and driving regimes for which comparatively long-lived prethermal regimes persist in a realistic setting. These arguments imply that Floquet control of quantum phases in solids entails a twofold challenge: engineering (i) a prethermal Hamiltonian that captures the desired dynamics on sufficiently long timescales, and (ii) an electronic distribution with respect to this prethermal Hamiltonian on the same timescales.

A complementary route to Floquet engineering aims to exploit dissipation in open systems to stabilize a driven steady state at long times, for which the energy influx absorbed from the pump is compensated for by energy dissipation into the environment (Esin et al., 2018; Seetharam et al., 2019). In a solid-state setting, the crystal lattice can act as a thermostat for the electronic system due to the large timescale separation between electronic and lattice dynamics, with early works suggesting routes toward the controlled dissipative population of single-particle Floquet states (Dehghani, Oka, and Mitra, 2014; Iadecola, Neupert, and Chamon, 2015; Seetharam et al., 2015). The phase diagrams of infinite-time steady states of clean systems have been established to exhibit rich phase transitions (Mitra et al., 2006; Mendoza-Arenas et al., 2017; Peronaci, Schiro, and Parcollet, 2018; Walldorf et al., 2019; Klöckner, Karrasch, and Kennes, 2020; Kalthoff et al., 2021), which are, however, expected to be first order at finite driving frequency (Mathey and Diehl, 2019).

\section{Engineering correlated systems}

An interesting prospect concerns the utilization of tailored light pulses to engineer novel phases of matter in correlated electron systems. The use of light to modulate interactions or selectively break symmetries to tune the interplay of competing phases promises a rich playground to stabilize new phases of matter but remains a largely unexplored and methodologically challenging regime. We now discuss a few different examples.

Magnetic Mott insulators have recently emerged as a promising class of candidate materials for Floquet engineering in correlated systems (Mentink, Balzer, and Eckstein, 2015; Bukov, Kolodrubetz, and Polkovnikov, 2016; Claassen et al., 2017; Kennes et al., 2018; Walldorf et al., 2019). In a Mott insulator, strong local Coulomb repulsion, typically in localized transition-metal $d$ orbitals, freezes local charge degrees of freedom at commensurate filling to open an insulating gap and form local magnetic moments. If the pump frequency is sufficiently red detuned from the charge gap, energy cannot be absorbed resonantly as photons couple to charge [Figs. 12(a) and 12(b)]. While the charge sector remains inert on short timescales, a prethermal regime can emerge that is characterized by transiently modified effective magnetic interactions between the local moments (Mentink, Balzer, and Eckstein, 2015; Claassen et al., 2017). 
A minimal model to describe these effects is the driven halffilled single-band Hubbard model

$$
\hat{H}(t)=-t_{\mathrm{h}} \sum_{\langle i j\rangle \sigma} e^{i e \mathbf{A}(t) \cdot \mathbf{r}_{i j} / \hbar} \hat{c}_{i \sigma}^{\dagger} \hat{c}_{j \sigma}+U \sum_{i} \hat{n}_{i \uparrow} \hat{n}_{i \downarrow},
$$

where $t_{\mathrm{h}}$ and $U$ denote the hopping of electrons between neighboring sites and local Coulomb repulsion, respectively. The optical pump enters via minimal coupling to a gauge field $\mathbf{A}(t)$.

At equilibrium, the half-filled Hubbard model at low energies and large $U$ canonically maps onto effective nearest-neighbor Heisenberg interactions $J \approx 4 t_{\mathrm{h}}^{2} / U$ that arise from virtual exchange. Suppose that the pump frequency $\omega$ is sufficiently red detuned from the Mott gap $\sim U-4 t_{\mathrm{h}}$. If the charge sector remains inert out of equilibrium, then a perturbative calculation, which simultaneously integrates out charge degrees of freedom and photons, leads to a transient photoinduced renormalization of spin-exchange interactions that is proportional to $J \approx \sum_{m} 4 t_{\mathrm{h}}^{2}\left[J_{m}(F)\right]^{2} /(U-m \hbar \omega)$ (Itin and Katsnelson, 2015; Mentink, Balzer, and Eckstein, 2015; Chaudhary, Hsieh, and Refael, 2019).

A physical picture readily emerges when one notes that the electron participating in the exchange process can now lower the Coulomb repulsion energy $U$ of the virtual intermediate state by absorbing a photon from the pump field, thereby enhancing the effective spin exchange. A schematic depiction of this process is shown in Fig. 12(c). Conversely, for strong pump fields, higher-order multiphoton processes can dominate and even permit the sign of the Heisenberg exchange to be flipped if $m$-photon processes with $m \hbar \omega>U$ dominate (Mentink, Balzer, and Eckstein, 2015). For a typical Mott antiferromagnet with $U \sim 2 \mathrm{eV}, t_{\mathrm{h}} \sim 0.1 \mathrm{eV}, a \sim 3 \AA$ (corresponding to $J \sim 20 \mathrm{meV}$ at equilibrium), and pumping at $700 \mathrm{~nm}$, one finds an enhancement of the exchange coupling in the range $\Delta J \sim 0.2$ to $20 \mathrm{meV}$ for a peak field strength of 0.05 to $0.5 \mathrm{~V}^{-1}$ (Batignani et al., 2015).

Frustrated quantum magnets, encompassing materials in which competing magnetic interactions render minimization of the free energy landscape nontrivial, are another interesting class to study. In these compounds, transient modifications of the magnetic interactions are expected to have a sizable effect. While the previously discussed role of overall renormalization of Heisenberg interactions is reflected solely in shifts of the excitation spectrum without affecting the ground state, strongly frustrated systems typically host rich phase diagrams of competing magnetic orders such that a small photoinduced transient change of competing subdominant interactions can nudge the system into a proximal phase (Kennes, Claassen et al., 2019). Moreover, such materials are highly sensitive to the breaking of symmetries, granting a powerful handle to steer the nonequilibrium phase on prethermal timescales, which can be kept sufficiently long via detuning from the Mott gap.

From these considerations, one avenue of utilizing Floquet engineering to transiently stabilize a novel correlated state of matter builds upon the observation that pumping a frustrated Mott insulator with circularly polarized light permits the selective breaking of time-reversal symmetry (TRS) and inversion without breaking the $\mathrm{SU}(2)$ spin-rotational symmetry of the magnetic moments (Claassen et al., 2017; Kitamura, Oka, and Aoki, 2017). While TRS can be readily broken at equilibrium via external magnetic fields, the dominant contribution to low-energy spin dynamics comes from a Zeeman splitting that breaks SU(2), quickly magnetizing the system. The role of the pump is to generate a transient photoinduced scalar spin chirality $\mathbf{S}_{i} \cdot\left(\mathbf{S}_{j} \times \mathbf{S}_{k}\right)$, whose form can be inferred from symmetry considerations alone and which microscopically arises from fourth-order virtual processes involving elementary triangles of the underlying lattice [Fig. 12(d)]. For example, it was shown that in kagome antiferromagnets, such as herbertsmithite, a weak optical pump tuned between one- and two-photon resonances of the parent Mott insulator can induce a transition to a proximal chiral spin liquid phase (Claassen et al., 2017), suggesting a novel nonequilibrium pathway to one of the earliest and most elusive candidates of a gapped quantum spin liquid with topological order.

Strong spin-orbit-coupled multiorbital compounds constitute a natural generalization of such a nonequilibrium separation of charge and spin degrees of freedom (Liu, Hejazi, and Balents, 2018; Hejazi, Liu, and Balents, 2019; Arakawa and Yonemitsu, 2021; Sriram and Claassen, 2021), which could allow for Floquet engineering while also mitigating heating. In the orthorhombic titanates, the partially filled $t_{2 g}$ manifold has been proposed as a prime target for optical manipulation of effective Kugel-Khomskii spin-orbital interactions. In Kitaev materials such as $\alpha-\mathrm{RuCl}_{3}$, Floquet engineering can modify the interplay between Kitaev and competing exchange interactions to manipulate the magnetic state and induce a quantum spin liquid. In charge-transfer insulators such as cuprates, photoinduced rotational symmetry breaking was proposed to engineer magnetic interactions and ultimately destabilize the Mott antiferromagnetic phase in favor of a $d$-wave superconductor (Kennes, Claassen et al., 2019). Conversely, theoretical predictions of spectroscopic signatures suggest that the controlled manipulation of magnetic exchange interactions could be observed in time-resolved Raman or resonant inelastic x-ray scattering (Wang, Claassen et al., 2018), for instance, via tracking the softening of bimagnon excitations (Wang, Devereaux, and Chen, 2018).

Fractional quantum Hall systems provide another intriguing direction to engineer prethermal correlated phases using external driving. While a vast series of Abelian filling fractions have been experimentally realized (Stormer, Tsui, and Gossard, 1999), more elusive fractional quantum Hall states (Moore and Read, 1991; Read and Rezayi, 1999) with non-Abelian quasiparticles are well known to prefer finetuned or multibody interactions for stabilization (Greiter, Wen, and Wilczek, 1991) while simultaneously incurring tremendous interest for potential applications in topological quantum computing and beyond (Nayak et al., 2008). In principle, Landau levels in ultraclean electron gases provide a natural separation of energy scales for Floquet engineering. If the pump frequency is sufficiently detuned from a cyclotron resonance, the regularity in Landau-level spacing guarantees the absence of multiphoton resonances at low photon number. If heating can be suppressed, such a setting has been predicted 
to give rise to photoinduced three-body interactions that arise from virtual inter-Landau-level scattering (Lee et al., 2018), suggesting a nonequilibrium route to stabilize a Moore-Read state. Conversely, resonant excitation between Landau levels has been proposed to engineer effective multilayer fractional quantum Hall models (Ghazaryan et al., 2017).

\section{OUTLOOK}

In this Colloquium, we discussed a set of results that provide insights into ultrafast phenomena in photoexcited quantum materials. These advances rely on an unprecedented range of optical excitations and a growing set of tools to probe and simulate nonthermal pathways toward control and functionality on ultrafast timescales. In particular, photoinduced phase transitions to thermally inaccessible states via short optical excitation or photon dressing constitute promising routes to achieving nonequilibrium functionality.

Recent studies of photoinduced phase transitions have provided a more detailed understanding of the relevant microscopic degrees of freedom and their nonthermal behavior and have revealed the interplay between these degrees of freedom in the time domain. The realization of new applications based on optical switching into metastable phases is already being explored. For instance, the photoinduced charge configuration change of $1 T-\mathrm{TaS}_{2}$ has emerged as a new avenue for memory devices in low-temperature circuitry (Mraz et al., 2021). Similarly, advances in optical switching of superconductors (Budden et al., 2021) could be integrated into future microelectronics. An expansion of the range of applications mandates the identification of further materials whose nonthermal energy landscape allows for efficient switching mechanisms in metastable states.

Creation and control of properties through light-matter coupling via Floquet engineering is another promising approach. To realize robust functionality and devices, it is necessary to understand the role of dissipation and identify strategies to mitigate heating and decoherence. As such, a firm understanding of the coupling to environmental degrees of freedom is necessary. Moreover, suitable candidate materials need to be identified that host appropriate electronic structures and interactions to minimize energy absorption at selected frequencies while still permitting a controlled modification of the salient electronic features.

To reach the final goal of new functionalities based on quantum dynamics far from equilibrium, it will be of increasing importance to (i) design materials tailored to enabling precise nonequilibrium control by integrating material synthesis and nonequilibrium experimentation synergistically, and (ii) establish further bridges between the existing ultrafast materials science community and adjacent research fields. For example, the young field of polaritonic chemistry is bridging nonequilibrium quantum chemistry, quantum optics, and nanoplasmonics (Ebbesen, 2016; Feist, Galego, and Garcia-Vidal, 2018; Flick, Rivera, and Narang, 2018; Ruggenthaler et al., 2018). Inspired in part by these efforts, cavity materials science is an emergent topic at the boundary between quantum optics and nonequilibrium materials science (Hübener et al., 2021; Juraschek et al., 2021). The key idea is the replacement of a strong laser with a few-photon state in a strongly light-matter-coupled cavity that enables control of the material properties. One possible route to cavity control could employ the quantum nature of photons for effective Floquet engineering without the need of intense laser fields (Sentef et al., 2020).

Controlled synthesis of van der Waals heterostructures has recently emerged as a versatile tool for establishing electronic and structural properties with unprecedented control and variety. In this context, twistronics (Moiré potential engineering by twisting adjacent layers) is set to play a crucial role. The twist angle provides a critical handle on designing electronic and structural properties that can be geared with great flexibility (Kennes et al., 2021). The relevant kinetic energy scales of the Moiré superlattice can be orders of magnitude smaller than in the bulk material, suggesting that external nonequilibrium perturbations can have an outsized effect in determining electronic phases. Combining ultrafast light-matter interaction with twist control of such band structures hence suggests a particularly promising route to nonequilibrium functionalization, with the first explorations already under way (Topp et al., 2019; Kennes et al., 2021; Rodriguez-Vega, Vogl, and Fiete, 2021). These efforts should be complemented by more traditional avenues of materials science, such as materials synthesis tailored specifically to questions relevant to nonequilibrium control. For example, a systematic exploration of engineerable free energy landscapes, which are particularly suitable for control via photoexcitation, in complex oxides is highly desirable.

These approaches expose the need for new paradigms of materials synthesis and predictions that address the requirements for ultrafast control of nonthermal states of matter. Theory needs to turn from toy models to realistic materials, not only to guide the process of directed materials synthesis but also to provide principles for how nonthermal pathways of ultrafast control can be obtained. To achieve this leap, inspiration can be drawn from other fields where such an integrated approach has already been established. Namely, an increased feedback between experimental characterization, theoretical understanding, and materials synthesis is a strategy that is successfully employed in many branches of quantum materials research, for instance, in the field of oxides (Coll et al., 2019), or in the study of quantum materials with angleresolved photoemission spectroscopy (Sobota, He, and Shen, 2021). This interdisciplinary approach requires additional effort, as a common language and concepts need to be developed for thinking about the underlying physics. Parts of such a program were laid out in this Colloquium. This is but one step along the long path of unifying themes and languages, with the goal of fostering the relatively new field of ultrafast quantum materials science.

\section{ACKNOWLEDGMENTS}

We are grateful to H. Aoki, R. Averitt, M. Bonitz, A. Cavalleri, M. Dean, P. Hofmann, D. Juraschek, P. Kirchmann, A. Kogar, W.-S. Lee, D. Mazzone, M. Mitrano, P. Narang, D. Nicoletti, B. Normand, H. Petek, J. Ravnik, G. Refael, R. Tuovinen, and A. Zong for stimulating discussions and critical feedback. D. M. K, J. W. M, and M. A. S. acknowledge support from the Max Planck-New York City Center for 
Non-Equilibrium Quantum Phenomena. D. M. K. acknowledges the Deutsche Forschungsgemeinschaft (DFG, German Research Foundation) for support through RTG 1995 and under Germany's Excellence Strategy-Cluster of Excellence Matter and Light for Quantum Computing (ML4Q) EXC 2004/1-390534769. M.C. acknowledges support from a startup grant from the University of Pennsylvania, and from the Flatiron Institute, a division of the Simons Foundation. J. W. M. acknowledges support from the Cluster of Excellence "CUI: Advanced Imaging of Matter" of the DFG, EXC 2056, Project ID No. 390715994 and is funded by DFG, SFB-925, Project No. 170620586. M. A.S. acknowledges financial support from the DFG via the Emmy Noether Program (Grant No. SE 2558/2).

A. d.1. T, D. M. K., M. C., S. G., J. W. M., and M. A. S. contributed equally to this work.

\section{APPENDIX: TECHNICAL ADVANCES SHAPING ULTRAFAST QUANTUM MATERIALS SCIENCE}

\section{Experimental tools}

Time-resolved optical spectroscopy remains the most commonly used approach to access the time-dependent optical properties of quantum materials after photoexcitation. In recent years, the field has seen an evolution from the early measurements of transient absorption and reflectivity (Elsayed-Ali et al., 1987; Schoenlein et al., 1987; Koshihara et al., 1990; Miyano et al., 1997; Chemla and Shah, 2000; Tsen, 2001) into a multifaceted set of techniques where the particular details of experiments depend on the targeted subsystem and dynamics (Averitt and Taylor, 2002; Orenstein, 2012). Some of the most advantageous capabilities of these approaches are as follows: (i) direct detection of transient changes in the electronic joint density of states via frequency-resolved measurements of the transient complex optical conductivity from the terahertz to the extreme ultraviolet range (Sie et al., 2015; Jager et al., 2017; Siegrist et al., 2019; Baldini et al., 2020), (ii) simultaneous measurements of the dynamics of different subsystems taken by combining multiple detection schemes (von Hoegen et al., 2018), including transient nonlinear optical processes (Woerner et al., 2013; Sala et al., 2016; Mahmood et al., 2021) and polarization rotations that are sensitive to changes in magnetic orders (Beaurepaire et al., 1996; Kirilyuk, Kimel, and Rasing, 2010; Walowski and Münzenberg, 2016; Němec et al., 2018; Schlauderer et al., 2019; Kimel et al., 2020), (iii) the integrability with other external stimuli such as magnetic fields and hydrostatic pressure (Trigo et al., 2012; Mitrano et al., 2014; Cantaluppi et al., 2018; Nicoletti et al., 2018), and (iv) the ability to modulate and control the optical pulse to gain real-space information (Gedik et al., 2003; Torchinsky et al., 2013; Mahmood et al., 2018). These techniques have enabled the observation of a wide range of phenomena in the time domain, including quasiparticle relaxation dynamics, electron-boson coupling strengths, gap magnitudes, photoexcited order parameters, and collective mode oscillations (Brorson et al., 1990; Demsar, Biljaković, and Mihailovic, 1999; Demsar et al., 2002; Segre et al., 2002), phase coexistence, light-induced phase transitions (Koshihara et al.,
1990; Rini et al., 2007; Yusupov, Mertelj et al., 2010; Giannetti et al., 2016), magnetic moment precession (Nova et al., 2017; Disa et al., 2020; Afanasiev et al., 2021; Stupakiewicz et al., 2021) and the relaxation dynamics of superconductors, and light-induced superconductinglike states (Yu et al., 1991; Demsar et al., 1999; Kaindl et al., 2000; Fausti et al., 2011; Hu et al., 2014; Nicoletti et al., 2014; Mitrano et al., 2016; Cremin et al., 2019).

Time-resolved scattering techniques.-Ultrafast X-ray scattering techniques take advantage of photon wavelengths of comparable magnitude to the atomic spacing to probe structural dynamics (Cavalleri et al., 2006; Fritz et al., 2007; Johnson et al., 2008; Mankowsky et al., 2014; Gerber et al., 2015, 2017; Rettig et al., 2015) or the evolution of spin, charge, and orbital electronic orders (Först et al., 2011; Johnson et al., 2012; Lee et al., 2012; Beaud et al., 2014; Kubacka et al., 2014; Dean et al., 2016; Mitrano et al., 2019) after optical excitation. The way toward subpicosecond x-ray pulses has been paved by laser-driven (Rischel et al., 1997) and hybrid laser-accelerator-based sources (Schoenlein et al., 1996, 2000), which in addition to diffraction (Cavalleri et al., 2006; Johnson et al., 2008) have also enabled spectroscopic measurements (Cavalleri et al., 2005; Stamm et al., 2007). The advent of x-ray free-electron lasers (FELs) (Emma et al., 2010; Seddon et al., 2017) has led to another burst of quantum materials studies, benefiting from femtosecond pulses of angstrom wavelength and increased brilliance. We refer to in-depth reviews on ultrafast X-ray scattering (Buzzi et al., 2018) and resonant inelastic x-ray scattering (Cao et al., 2019; Mitrano and Wang, 2020).

The wavelength tunability of pulsed x-ray sources is key to accessing different phenomena. For example, the "soft" x-ray regime (250-2000 eV) includes many strongly resonant elemental absorption edges, such as $3 d$ transition metals, which can be targeted to probe electronic properties. Although such resonances also exist in the "hard" $\mathrm{x}$-ray regime $(>5000 \mathrm{eV})$, their main use is to access structural properties and large portions of reciprocal space. X-ray FELs can also be used to access inelastic scattering channels in the time domain, for example, to map out energy- and momentum-resolved nonequilibrium phonon dispersions (Trigo et al., 2013; Zhu et al., 2015), the dynamics of magnetic correlations (Dean et al., 2016; Mazzone et al., 2021; Paris et al., 2021) and orbitals (Parchenko et al., 2020), as well as disordering during ultrafast phase transitions (Wall et al., 2018). The coherence of FEL light has also been used to directly probe fluctuating topological order via X-ray photon correlation spectroscopy (Seaberg et al., 2017).

Ultrafast electron diffraction (UED) is a complementary scattering technique that also directly measures ultrafast structural dynamics in solids (Siwick et al., 2003; Zewail, 2006; Baum and Zewail, 2007). The larger scattering cross section of electrons relative to $\mathrm{x}$-ray photons makes UED particularly suited to studying thin samples. New $\mathrm{MeV}$ electron sources (Weathersby et al., 2015), terahertz streaking (Kealhofer et al., 2016; Zhao et al., 2018), and pulse compression schemes (Kim et al., 2020; Qi et al., 2020) can now achieve $<100$ fs time resolution. Recent experiments have taken advantage of these capabilities to investigate lattice dynamics (Mannebach et al., 2015; Waldecker et al., 2017; Konstantinova et al., 2018) and electronic orders 
(Haupt et al., 2016; Le Guyader et al., 2017; Vogelgesang et al., 2018; Zong et al., 2018; Sie et al., 2019), as well as to study electron-phonon couplings (Harb et al., 2016; Waldecker et al., 2016; Stern et al., 2018; René de Cotret et al., 2019; Horstmann et al., 2020).

Time- and angle-resolved photoemission spectroscopy (trARPES) measures changes in the band structure and singleparticle spectral function of solids with momentum resolution (Haight, Silberman, and Lilie, 1988; Petek and Ogawa, 1997; Bovensiepen and Kirchmann, 2012; Smallwood, Kaindl, and Lanzara, 2016; Gedik and Vishik, 2017; Nicholson et al., 2018; Zhou et al., 2018; Lv, Qian, and Ding, 2019). This technique has been used to study decoherence effects in the excitation process (Höfer et al., 1997; Ogawa et al., 1997; Reutzel, Li, and Petek, 2019), to shed light on the physics of high-temperature superconductors (Smallwood et al., 2012; Avigo et al., 2013; Yang et al., 2014, 2019; Parham et al., 2017), to track the melting and recovery of charge-density wave orders (Perfetti et al., 2006; Schmitt et al., 2008; Hellmann et al., 2010, 2012; Rohwer et al., 2011; Rettig et al., 2016; Ligges et al., 2018), to directly probe excitonic states (Cui et al., 2014; Madéo et al., 2020), and to measure the relaxation dynamics of photocurrents (Reimann et al., 2018; Güdde and Höfer, 2021) and the coupling between electronic and lattice degrees of freedom (Gerber et al., 2017; Kemper et al., 2017; Na et al., 2019). tr-ARPES also permits the detection of transiently populated topological states (Sobota et al., 2012, 2013; Belopolski et al., 2017; Zhang et al., 2017), the observation of Floquet-Bloch states (Wang et al., 2013; Mahmood et al., 2016), and the identification of nonthermal electronic regimes (Gierz et al., 2013; Johannsen et al., 2013; Na et al., 2020). An interesting prospect is the implementation of new detection schemes extending timeand momentum-resolved microscopy to FELs (Kutnyakhov et al., 2020).

Time-resolved scanning probes.-Time-resolved scanning near-field optical microscopy (tr-SNOM) tracks photoinduced changes in the optical constants of materials on the $10 \mathrm{~nm}$ length scale with high temporal and spectral resolution (Eisele et al., 2014; Wagner et al., 2014). tr-SNOM has been used to investigate photoinduced insulator-to-metal transitions (Dönges et al., 2016; Huber et al., 2016) and image the propagation of plasmon polaritons in a variety of systems (Wagner et al., 2014; Ni et al., 2016; Huber et al., 2017). Ultrafast scanning tunneling microscopy (STM) is also gaining traction and probes quantum tunneling at the atomic length scale with subfemtosecond time resolution (Nunes and Freeman, 1993; Cocker et al., 2013; Garg and Kern, 2020). Ultrafast STM has been used to track carrier and spin dynamics in photoexcited semiconductors (Terada et al., 2010; Yoshida et al., 2014), the ultrafast vibrational motion of a single molecule (Cocker et al., 2016), and image surface plasmons in gold (Garg and Kern, 2020).

Time-resolved transport.-Microstructured devices incorporating laser-triggered photoconductive switches (Auston, 1975) have been used to investigate a variety of ultrafast transport phenomena, such as ballistic electron flow in carbon nanotubes (Zhong et al., 2008), helicity-dependent photocurrents in topological insulators (Kastl et al., 2015), and the transport properties of Floquet-Bloch states in graphene
(McIver et al., 2020). Ultrafast transport dynamics have also been probed in carbon nanotubes (Gabor et al., 2012), graphene (Sun et al., 2012), and various van der Waals heterostructures (Ma et al., 2016; Massicotte et al., 2016; Arp et al., 2019) by measuring the photocurrent generated in response to two time-delayed laser pulses.

\section{Theoretical tools}

Time-dependent density functional theory (TDDFT) is an extension of ground-state density functional theory, with similar merits and challenges (Marques et al., 2012; Ullrich, 2012). The full time-dependent Schrödinger equation for the many-particle wave function is replaced by an auxiliary set of Schrödinger (Kohn-Sham) equations, which determine the exact time-dependent density of the system (Runge and Gross, 1984). Coulomb interactions are accounted for via exchange-correlation potentials, although their exact forms are unknown in practice. TDDFT has been successfully applied to a host of systems in weak and strong external driving fields, ranging from atoms and molecules to periodic solids (De Giovannini et al., 2013; Lian et al., 2020). The strength of TDDFT lies in the ability to describe full-fledged material-specific details. However, the faithful description of nontrivial correlation effects still poses a severe challenge for TDDFT-based methods, particularly in pump-probe settings.

Nonequilibrium Green's functions and diagrammatic techniques provide a framework for obtaining few-body correlation functions of time-dependent problems without computing the actual many-body wave functions (Stefanucci and van Leeuwen, 2013). Green's function techniques are based on single-particle propagators that describe the probability amplitude for particles (electrons, phonons, etc.) to travel between two space-time points while interacting with the rest of the system. These many-body interactions are described through the many-body self-energy. Notable approximations to calculate the self-energy include the so-called $G W$ approximation for screened interactions (Aryasetiawan and Gunnarsson, 1998; Thygesen and Rubio, 2007), nonequilibrium dynamical mean-field theory (DMFT) for strongly correlated systems, including Mott insulators with mainly local self-energies (Freericks, Turkowski, and Zlatić, 2006; Tsuji, Oka, and Aoki, 2008; Aoki et al., 2014), and the real-time functional renormalization group approach (Kennes et al., 2012). In a nonequilibrium setup these approximation schemes are more difficult to handle than at equilibrium because timetranslational invariance cannot be exploited and, in general, two time variables instead of one energy variable must be kept. Nonequilibrium Green's functions are an ideal starting point when approximations for the self-energy can be physically motivated, e.g., perturbative weak- or strong-coupling expansions or the local-self-energy approximation of DMFT.

Green's function-based real-time diagrammatic quantum Monte Carlo techniques provide alternative and a priori controlled solutions via stochastic sampling of a perturbation series. While these approaches suffer from a notorious "dynamical sign problem" (Werner, Oka, and Millis, 2009) whereby the nonpositivity of sampling weights results in a computational effort that increases exponentially in time, recent algorithmic advances with modest computational 
scaling, including the "inchworm" algorithm (Cohen et al., 2015) and conformal transformations (Bertrand et al., 2019), have been put forward. Real-time diagrammatic quantum Monte Carlo calculation is the method of choice whenever the sign problem can be averted.

Tensor networks provide a faithful representation of manybody states and operators as a "contraction" network of tensors that encode the local properties of the system (Verstraete, Murg, and Cirac, 2008; Schollwöck, 2011). In one dimension matrix product states are a particularly prominent example of a tensor network and provide the basis for elegant density matrix renormalization group implementations (Schollwöck, 2011). In general, the representation of generic quantum states requires one to contract tensor dimensions that scale with the exponential size of the full many-body Hilbert space. However, in many physically relevant situations the notion of locality introduced by the tensor network allows one to represent states with low entanglement via tensors of much reduced dimensionality. In this sense, tensor networks might be viewed as a low-entanglement method. The entanglement of ground states generally scales favorably in low-dimensional systems due to the area law (Eisert, Cramer, and Plenio, 2010; Orús, 2014). Conversely, dynamics far from equilibrium typically involves highly excited states with volume law scaling of the entanglement entropy. Therefore, in nonequilibrium studies only short timescales can be simulated. Numerous methods for time evolution have been proposed and benchmarked, including the block decimation, matrix product operator techniques, Krylov methods, or the timedependent variational principle (Paeckel et al., 2019). In a nutshell, tensor networks are useful whenever entanglement entropy can be kept at bay.

Other variational techniques.-Beyond asymptotically exact representations of the many-body wave function, simpler variational starting points serve as theoretical tools to gain insight into specific physical systems, often based on some intuition of the relevant physics. Examples include the timedependent Gutzwiller approximation (Seibold and Lorenzana, 2001; Schiró and Fabrizio, 2010), variational Monte Carlo calculations (Carleo et al., 2012), Gaussian and non-Gaussian variational states (Shi, Demler, and Cirac, 2018; Hackl et al., 2020), and Gross-Pitaevskii equations (Gross, 1961; Pitaevskii, 1961). More recently, machine-learning-inspired restricted Boltzmann machines have emerged that provide a more flexible variational subspace for time evolution (Carleo and Troyer, 2017). To summarize, common to all these techniques are Ansätze for the many-body wave function with an economical number of free parameters, which are governed by equations of motion as determined via the time-dependent variational principle. Although the general usefulness of these techniques for simulations related to pump-probe experiments is not yet clear, they can provide a good starting point whenever a suitable variational manifold can be identified.

\section{REFERENCES}

Abanin, D., W. De Roeck, W. W. Ho, and F. Huveneers, 2017a, "A rigorous theory of many-body prethermalization for periodically driven and closed quantum systems," Commun. Math. Phys. 354, 809.

Abanin, D. A., W. De Roeck, Wen W. Ho, and F. Huveneers, 2017b, "Effective Hamiltonians, prethermalization, and slow energy absorption in periodically driven many-body systems," Phys. Rev. B 95, 014112.

Abanin, D. A., W. De Roeck, and F. Huveneers, 2015, "Exponentially Slow Heating in Periodically Driven Many-Body Systems," Phys. Rev. Lett. 115, 256803.

Aeschlimann, S., et al., 2021, "Survival of Floquet-Bloch states in the presence of scattering," Nano Lett. 21, 5028.

Afanasiev, D., J. R. Hortensius, B. A. Ivanov, A. Sasani, E. Bousquet, Y. M. Blanter, R. V. Mikhaylovskiy, A. V. Kimel, and A.D. Caviglia, 2021, "Ultrafast control of magnetic interactions via light-driven phonons," Nat. Mater. 20, 607.

Alexandradinata, A., et al., 2020, "The future of the correlated electron problem," arXiv:2010.00584.

Allen, P. B., 1987, "Theory of Thermal Relaxation of Electrons in Metals,” Phys. Rev. Lett. 59, 1460.

Anisimov, S. I., B. L. Kapeliovich, and T. L. Perelman, 1974, "Electron emission from metal surfaces exposed to ultrashort laser pulses," Sov. Phys. JETP 39, 375, http://jetp.ras.ru/cgi-bin/e/index/ e/39/2/p375?a=list.

Aoki, H., N. Tsuji, M. Eckstein, M. Kollar, T. Oka, and P. Werner, 2014, "Nonequilibrium dynamical mean-field theory and its applications," Rev. Mod. Phys. 86, 779.

Arakawa, N., and K. Yonemitsu, 2021, "Floquet engineering of mott insulators with strong spin-orbit coupling," Phys. Rev. B 103, L100408.

Arp, T. B., D. Pleskot, V. Aji, and N. M. Gabor, 2019, "Electron-hole liquid in a van der Waals heterostructure photocell at room temperature," Nat. Photonics 13, 245.

Aryasetiawan, F., and O. Gunnarsson, 1998, "The $G W$ method," Rep. Prog. Phys. 61, 237.

Asamitsu, A., Y. Tomioka, H. Kuwahara, and Y. Tokura, 1997, "Current switching of resistive states in magnetoresistive manganites," Nature (London) 388, 50.

Auston, D. H., 1975, "Picosecond optoelectronic switching and gating in silicon,” Appl. Phys. Lett. 26, 101.

Autler, S. H., and C. H. Townes, 1955, "Stark effect in rapidly varying fields," Phys. Rev. 100, 703.

Averitt, R. D., and A. J. Taylor, 2002, "Ultrafast optical and farinfrared quasiparticle dynamics in correlated electron materials," J. Phys. Condens. Matter 14, R1357.

Avigo, I., et al., 2013, "Coherent excitations and electron-phonon coupling in $\mathrm{Ba} / \mathrm{EuFe}_{2} \mathrm{As}_{2}$ compounds investigated by femtosecond time- and angle-resolved photoemission spectroscopy," J. Phys. Condens. Matter 25, 094003.

Babadi, M., M. Knap, I. Martin, G. Refael, and E. Demler, 2017, "Theory of parametrically amplified electron-phonon superconductivity," Phys. Rev. B 96, 014512.

Baldini, E., et al., 2020, "Electron-phonon-driven three-dimensional metallicity in an insulating cuprate," Proc. Natl. Acad. Sci. U.S.A. 117, 6409.

Balzer, K., F. A. Wolf, I. P. McCulloch, P. Werner, and M. Eckstein, 2015, "Nonthermal Melting of Néel Order in the Hubbard Model," Phys. Rev. X 5, 031039.

Bartels, R. A., A. Paul, H. Green, H. C. Kapteyn, M. M. Murnane, S. Backus, I. P. Christov, Y. Liu, D. Attwood, and C. Jacobsen, 2002, "Generation of spatially coherent light at extreme ultraviolet wavelengths," Science 297, 376.

Basov, D. N., R. D. Averitt, and D. Hsieh, 2017, “Towards properties on demand in quantum materials," Nat. Mater. 16, 1077. 
Basov, D. N., R. D. Averitt, D. van der Marel, M. Dressel, and K. Haule, 2011, "Electrodynamics of correlated electron materials," Rev. Mod. Phys. 83, 471.

Batignani, G., D. Bossini, N. Di Palo, C. Ferrante, E. Pontecorvo, G. Cerullo, A. Kimel, and T. Scopigno, 2015, "Probing ultrafast photo-induced dynamics of the exchange energy in a Heisenberg antiferromagnet," Nat. Photonics 9, 506.

Bauer, M., A. Marienfeld, and M. Aeschlimann, 2015, "Hot electron lifetimes in metals probed by time-resolved two-photon photoemission," Prog. Surf. Sci. 90, 319.

Baum, P., D.-S. Yang, and A. H. Zewail, 2007, “4D visualization of transitional structures in phase transformations by electron diffraction," Science 318, 788.

Baum, P., and A. H. Zewail, 2007, "Attosecond electron pulses for 4D diffraction and microscopy," Proc. Natl. Acad. Sci. U.S.A. 104, 18409.

Beaud, P., et al., 2014, "A time-dependent order parameter for ultrafast photoinduced phase transitions," Nat. Mater. 13, 923.

Beaulieu, S., et al., 2021, "Ultrafast dynamical Lifshitz transition," Sci. Adv. 7, eabd9275.

Beaurepaire, E., J.-C. Merle, A. Daunois, and J.-Y. Bigot, 1996, "Ultrafast Spin Dynamics in Ferromagnetic Nickel," Phys. Rev. Lett. 76, 4250.

Becker, M. F., A. B. Buckman, R. M. Walser, T. Lépine, P. Georges, and A. Brun, 1994, "Femtosecond laser excitation of the semiconductor-metal phase transition in $\mathrm{VO}_{2}$," Appl. Phys. Lett. 65, 1507.

Belopolski, I., et al., 2017, "Signatures of a time-reversal symmetric Weyl semimetal with only four Weyl points," Nat. Commun. 8, 942.

Bertrand, C., S. Florens, O. Parcollet, and X. Waintal, 2019, "Reconstructing Nonequilibrium Regimes of Quantum ManyBody Systems from the Analytical Structure of Perturbative Expansions," Phys. Rev. X 9, 041008.

Bonitz, M., 2016, Quantum Kinetic Theory (Springer International Publishing, Basel).

Bovensiepen, U., and P. S. Kirchmann, 2012, "Elementary relaxation processes investigated by femtosecond photoelectron spectroscopy of two-dimensional materials," Laser Photonics Rev. 6, 589.

Bray, A. J., 1994, "Theory of phase-ordering kinetics," Adv. Phys. 43, 357.

Brorson, S. D., A. Kazeroonian, J. S. Moodera, D. W. Face, T. K. Cheng, E. P. Ippen, M. S. Dresselhaus, and G. Dresselhaus, 1990, "Femtosecond Room-Temperature Measurement of the ElectronPhonon Coupling Constant $\gamma$ in Metallic Superconductors," Phys. Rev. Lett. 64, 2172.

Bucksbaum, P. H., A. Zavriyev, H. G. Muller, and D. W. Schumacher, 1990, "Softening of the $\mathrm{H}_{2}^{+}$Molecular Bond in Intense Laser Fields," Phys. Rev. Lett. 64, 1883.

Budden, M., et al., 2021, "Evidence for metastable photo-induced superconductivity in $\mathrm{K}_{3} \mathrm{C}_{60}$," Nat. Phys. 17, 611 .

Bukov, M., L. D’Alessio, and A. Polkovnikov, 2015, "Universal high-frequency behavior of periodically driven systems: From dynamical stabilization to Floquet engineering," Adv. Phys. 64, 139.

Bukov, M., M. Kolodrubetz, and A. Polkovnikov, 2016, "SchriefferWolff Transformation for Periodically Driven Systems: Strongly Correlated Systems with Artificial Gauge Fields," Phys. Rev. Lett. 116, 125301.

Buzzi, M., M. Först, R. Mankowsky, and A. Cavalleri, 2018, "Probing dynamics in quantum materials with femtosecond X-rays," Nat. Rev. Mater. 3, 299.
Buzzi, M., et al., 2020, "Photomolecular High-Temperature Superconductivity," Phys. Rev. X 10, 031028.

Cantaluppi, A., M. Buzzi, G. Jotzu, D. Nicoletti, M. Mitrano, D. Pontiroli, M. Riccò, A. Perucchi, P. Di Pietro, and A. Cavalleri, 2018, "Pressure tuning of light-induced superconductivity in $\mathrm{K}_{3} \mathrm{C}_{60}$," Nat. Phys. 14, 837.

Cao, Y., D. G. Mazzone, D. Meyers, J. P. Hill, X. Liu, S. Wall, and M.P. M. Dean, 2019, "Ultrafast dynamics of spin and orbital correlations in quantum materials: An energy- and momentumresolved perspective," Phil. Trans. R. Soc. A 377, 20170480.

Carleo, G., F. Becca, M. Schiró, and M. Fabrizio, 2012, "Localization and glassy dynamics of many-body quantum systems," Sci. Rep. 2, 243.

Carleo, G., and M. Troyer, 2017, "Solving the quantum many-body problem with artificial neural networks," Science 355, 602.

Cavalleri, A., Th. Dekorsy, H. H. W. Chong, J. C. Kieffer, and R. W. Schoenlein, 2004, "Evidence for a structurally-driven insulatorto-metal transition in $\mathrm{VO}_{2}$ : A view from the ultrafast timescale," Phys. Rev. B 70, 161102.

Cavalleri, A., M. Rini, H. H. W. Chong, S. Fourmaux, T. E. Glover, P. A. Heimann, J. C. Kieffer, and R. W. Schoenlein, 2005, "BandSelective Measurements of Electron Dynamics in $\mathrm{VO}_{2}$ Using Femtosecond Near-Edge X-Ray Absorption," Phys. Rev. Lett. 95, 067405.

Cavalleri, A., C. Tóth, C. W. Siders, J. A. Squier, F. Ráksi, P. Forget, and J. C. Kieffer, 2001, "Femtosecond Structural Dynamics in $\mathrm{VO}_{2}$ during an Ultrafast Solid-Solid Phase Transition," Phys. Rev. Lett. 87, 237401.

Cavalleri, A., S. Wall, C. Simpson, E. Statz, D. W. Ward, K. A. Nelson, M. Rini, and R. W. Schoenlein, 2006, "Tracking the motion of charges in a terahertz light field by femtosecond x-ray diffraction," Nature (London) 442, 664.

Cerullo, G., and S. De Silvestri, 2003, "Ultrafast optical parametric amplifiers," Rev. Sci. Instrum. 74, 1.

Chaudhary, S., D. Hsieh, and G. Refael, 2019, "Orbital Floquet engineering of exchange interactions in magnetic materials," Phys. Rev. B 100, 220403.

Chemla, D. S., and J. Shah, 2000, "Ultrafast dynamics of many-body processes and fundamental quantum mechanical phenomena in semiconductors," Proc. Natl. Acad. Sci. U.S.A. 97, 2437.

Chiocchetta, A., A. Gambassi, S. Diehl, and J. Marino, 2017, "Dynamical Crossovers in Prethermal Critical States," Phys. Rev. Lett. 118, 135701.

Chou, Y.-Z, Y. Liao, and M. S. Foster, 2017, "Twisting Anderson pseudospins with light: Quench dynamics in terahertz-pumped BCS superconductors," Phys. Rev. B 95, 104507.

Chu, H., et al., 2020, "Phase-resolved Higgs response in superconducting cuprates," Nat. Commun. 11, 1793.

Chuang, Y. D., et al., 2013, "Real-Time Manifestation of Strongly Coupled Spin and Charge Order Parameters in Stripe-Ordered $\mathrm{La}_{1.75} \mathrm{Sr}_{0.25} \mathrm{NiO}_{4}$ Nickelate Crystals Using Time-Resolved Resonant X-Ray Diffraction,” Phys. Rev. Lett. 110, 127404.

Claassen, M., 2021, "Flow renormalization and emergent prethermal regimes of periodically-driven quantum systems," arXiv:2103 .07485 .

Claassen, M., C. Jia, B. Moritz, and T. P. Devereaux, 2016, "Alloptical materials design of chiral edge modes in transition-metal dichalcogenides," Nat. Commun. 7, 13074.

Claassen, M., H.-C. Jiang, B. Moritz, and T. P. Devereaux, 2017, "Dynamical time-reversal symmetry breaking and photo-induced chiral spin liquids in frustrated Mott insulators," Nat. Commun. 8, 1192. 
Claassen, M., D. M. Kennes, M. Zingl, M. A. Sentef, and A. Rubio, 2019, "Universal optical control of chiral superconductors and Majorana modes," Nat. Phys. 15, 766.

Cocker, T. L., V. Jelic, M. Gupta, S. J. Molesky, J. A. J. Burgess, G. De Los Reyes, L. V. Titova, Y. Y. Tsui, M. R. Freeman, and F. A. Hegmann, 2013, "An ultrafast terahertz scanning tunnelling microscope," Nat. Photonics 7, 620.

Cocker, T. L., D. Peller, P. Yu, J. Repp, and R. Huber, 2016, "Tracking the ultrafast motion of a single molecule by femtosecond orbital imaging," Nature (London) 539, 263.

Cohen, G., E. Gull, D. R. Reichman, and A. J. Millis, 2015, “Taming the Dynamical Sign Problem in Real-Time Evolution of Quantum Many-Body Problems," Phys. Rev. Lett. 115, 266802.

Coll, M., et al., 2019, “Towards oxide electronics: A roadmap," Appl. Surf. Sci. 482, 1.

Coulthard, J. R., S. R. Clark, S. Al-Assam, A. Cavalleri, and D. Jaksch, 2017, "Enhancement of superexchange pairing in the periodically driven Hubbard model," Phys. Rev. B 96, 085104.

Cremin, K. A., J. Zhang, C. C. Homes, G. D. Gu, Z. Sun, M. M. Fogler, A. J. Millis, D. N. Basov, and R. D. Averitt, 2019, "Photoenhanced metastable $c$-axis electrodynamics in stripe-ordered cuprate $\mathrm{La}_{1.885} \mathrm{Ba}_{0.115} \mathrm{CuO}_{4}$," Proc. Natl. Acad. Sci. U.S.A. 116, 19875.

Cui, X., C. Wang, A. Argondizzo, S. Garrett-Roe, B. Gumhalter, and H. Petek, 2014, "Transient excitons at metal surfaces," Nat. Phys. 10, 505 .

D'Alessio, L., and A. Polkovnikov, 2013, "Many-body energy localization transition in periodically driven systems," Ann. Phys. (Amsterdam) 333, 19

D'Alessio, L., and M. Rigol, 2014, "Long-time Behavior of Isolated Periodically Driven Interacting Lattice Systems," Phys. Rev. X 4, 041048.

Danz, T., T. Domröse, and C. Ropers, 2021, "Ultrafast nanoimaging of the order parameter in a structural phase transition," Science 371, 371.

Dasari, N., and M. Eckstein, 2018, "Transient Floquet engineering of superconductivity," Phys. Rev. B 98, 235149.

Dasari, N., and M. Eckstein, 2019, "Ultrafast electric field controlled spin correlations in the Hubbard model," Phys. Rev. B 100, 121114.

Dean, M. P. M., et al., 2016, "Ultrafast energy- and momentumresolved dynamics of magnetic correlations in the photo-doped Mott insulator $\mathrm{Sr}_{2} \mathrm{IrO}_{4}$," Nat. Mater. 15, 601 .

Decker, K. S. C., C. Karrasch, J. Eisert, and D. M. Kennes, 2020, "Floquet Engineering Topological Many-Body Localized Systems," Phys. Rev. Lett. 124, 190601.

De Giovannini, U., G. Brunetto, A. Castro, J. Walkenhorst, and A. Rubio, 2013, "Simulating pump-probe photoelectron and absorption spectroscopy on the attosecond timescale with time-dependent density functional theory," ChemPhysChem 14, 1363.

Dehghani, H., M. Hafezi, and P. Ghaemi, 2021, "Light-induced topological superconductivity via Floquet interaction engineering," Phys. Rev. Research 3, 023039.

Dehghani, H., and A. Mitra, 2017, "Dynamical generation of superconducting order of different symmetries in hexagonal lattices,” Phys. Rev. B 96, 195110.

Dehghani, H., T. Oka, and A. Mitra, 2014, "Dissipative Floquet topological systems," Phys. Rev. B 90, 195429.

Dehghani, H., T. Oka, and A. Mitra, 2015, "Out-of-equilibrium electrons and the Hall conductance of a Floquet topological insulator," Phys. Rev. B 91, 155422.

Demsar, J., 2020, "Non-equilibrium phenomena in superconductors probed by femtosecond time-domain spectroscopy," J. Low Temp. Phys. 201, 676.
Demsar, J., K. Biljaković, and D. Mihailovic, 1999, "Single Particle and Collective Excitations in the One-Dimensional Charge Density Wave Solid $\mathrm{K}_{0.3} \mathrm{MoO}_{3}$ Probed in Real Time by Femtosecond Spectroscopy," Phys. Rev. Lett. 83, 800.

Demsar, J., L. Forró, H. Berger, and D. Mihailovic, 2002, "Femtosecond snapshots of gap-forming charge-density-wave correlations in quasi-two-dimensional dichalcogenides $1 \mathrm{~T}-\mathrm{TaS}_{2}$ and $2 \mathrm{H}$-TaS $\mathrm{Ta}_{2}$," Phys. Rev. B 66, 041101.

Demsar, J., D. Mihailovic, V. V. Kabanov, and K. Biljakovic, 2004, "Ultrafast real-time spectroscopy of low dimensional charge density wave compounds," in Molecular Nanowires and Other Quantum Objects, edited by A. S. Alexandrov, J. Demsar, and I. K. Yanson (Springer Netherlands, Dordrecht), p. 377.

Demsar, J., B. Podobnik, V. V. Kabanov, Th. Wolf, and D. Mihailovic, 1999, "Superconducting Gap $\Delta_{c}$, the Pseudogap $\Delta_{p}$, and Pair Fluctuations above $T_{c}$ in Overdoped $\mathrm{Y}_{1-x} \mathrm{Ca}_{x} \mathrm{Ba}_{2} \mathrm{Cu}_{3} \mathrm{O}_{7-\delta}$ from Femtosecond Time-Domain Spectroscopy,' Phys. Rev. Lett. 82, 4918.

Denny, S. J., S. R. Clark, Y. Laplace, A. Cavalleri, and D. Jaksch, 2015, "Proposed Parametric Cooling of Bilayer Cuprate Superconductors by Terahertz Excitation,” Phys. Rev. Lett. 114, 137001.

Disa, A. S., M. Fechner, T. F. Nova, B. Liu, M. Först, D. Prabhakaran, P. G. Radaelli, and A. Cavalleri, 2020, "Polarizing an antiferromagnet by optical engineering of the crystal field," Nat. Phys. 16, 937.

Dolgirev, P. E., M. H. Michael, A. Zong, N. Gedik, and E. Demler, 2020, "Self-similar dynamics of order parameter fluctuations in pump-probe experiments," Phys. Rev. B 101, 174306.

Dönges, S. A., O. Khatib, B. T. O'Callahan, J. M. Atkin, J. H. Park, D. Cobden, and M. B. Raschke, 2016, "Ultrafast nanoimaging of the photoinduced phase transition dynamics in $\mathrm{VO}_{2}$," Nano Lett. 16, 3029.

Dunlap, D. H., and V. M. Kenkre, 1986, "Dynamic localization of a charged particle moving under the influence of an electric field," Phys. Rev. B 34, 3625.

Ebbesen, T. W., 2016, "Hybrid light-matter states in a molecular and material science perspective," Acc. Chem. Res. 49, 2403.

Eisele, M., T. L. Cocker, M. A. Huber, M. Plankl, L. Viti, D. Ercolani, L. Sorba, M. S. Vitiello, and R. Huber, 2014, "Ultrafast multiterahertz nano-spectroscopy with sub-cycle temporal resolution," Nat. Photonics 8, 841.

Eisert, J., M. Cramer, and M. B. Plenio, 2010, "Area laws for the entanglement entropy,” Rev. Mod. Phys. 82, 277.

Eissing, A. K., V. Meden, and D. M. Kennes, 2016, "Renormalization in Periodically Driven Quantum Dots," Phys. Rev. Lett. 116, 026801.

Elsayed-Ali, H. E., T. B. Norris, M. A. Pessot, and G. A. Mourou, 1987, "Time-Resolved Observation of Electron-Phonon Relaxation in Copper," Phys. Rev. Lett. 58, 1212.

Emma, P., et al., 2010, "First lasing and operation of an ångstromwavelength free-electron laser," Nat. Photonics 4, 641.

Esin, I., M.S. Rudner, G. Refael, and N.H. Lindner, 2018, "Quantized transport and steady states of Floquet topological insulators," Phys. Rev. B 97, 245401.

Farrell, A., and T. Pereg-Barnea, 2016, "Edge-state transport in Floquet topological insulators," Phys. Rev. B 93, 045121.

Fausti, D., R. I. Tobey, N. Dean, S. Kaiser, A. Dienst, M. C. Hoffmann, S. Pyon, T. Takayama, H. Takagi, and A. Cavalleri, 2011, "Light-induced superconductivity in a stripe-ordered cuprate," Science 331, 189.

Fazekas, P., and E. Tosatti, 1979, "Electrical, structural and magnetic properties of pure and doped $1 T-\mathrm{TaS}_{2}$," Philos. Mag. B 39, 229. 
Feist, J., J. Galego, and F. J. Garcia-Vidal, 2018, "Polaritonic chemistry with organic molecules," ACS Photonics 5, 205.

Flick, J., N. Rivera, and P. Narang, 2018, "Strong light-matter coupling in quantum chemistry and quantum photonics," Nanophotonics 7, 1479.

Floquet, G., 1883, "On linear differential equations with periodic coefficients," Ann. Sci. Ec. Norm. Super. Ser. 2, 12, 47.

Först, M., C. Manzoni, S. Kaiser, Y. Tomioka, Y. Tokura, R. Merlin, and A. Cavalleri, 2011, "Nonlinear phononics as an ultrafast route to lattice control," Nat. Phys. 7, 854.

Först, M., et al., 2011, "Driving magnetic order in a manganite by ultrafast lattice excitation," Phys. Rev. B 84, 241104.

Freericks, J. K., V. M. Turkowski, and V. Zlatić, 2006, "Nonequilibrium Dynamical Mean-Field Theory," Phys. Rev. Lett. 97, 266408.

Fritz, D. M., et al., 2007, "Ultrafast bond softening in bismuth: Mapping a solid's interatomic potential with x-rays," Science $\mathbf{3 1 5}$, 633.

Fröhlich, D., A. Nöthe, and K. Reimann, 1985, “Observation of the Resonant Optical Stark Effect in a Semiconductor," Phys. Rev. Lett. $\mathbf{5 5}, 1335$.

Gabor, N. M., Z. Zhong, K. Bosnick, and P. L. McEuen, 2012, "Ultrafast Photocurrent Measurement of the Escape Time of Electrons and Holes from Carbon Nanotube $p-i-n$ Photodiodes," Phys. Rev. Lett. 108, 087404.

Garg, M., and K. Kern, 2020, "Attosecond coherent manipulation of electrons in tunneling microscopy," Science 367, 411.

Gedik, N., J. Orenstein, R. Liang, D. A. Bonn, and W. N. Hardy, 2003, "Diffusion of nonequilibrium quasi-particles in a cuprate superconductor," Science 300, 1410.

Gedik, N., and I. Vishik, 2017, "Photoemission of quantum materials," Nat. Phys. 13, 1029.

Gerasimenko, Y. A., I. Vaskivskyi, M. Litskevich, J. Ravnik, J. Vodeb, M. Diego, V. Kabanov, and D. Mihailovic, 2019, "Quantum jamming transition to a correlated electron glass in $1 T-\mathrm{TaS}_{2}$," Nat. Mater. 18, 1078.

Gerber, S., et al., 2015, "Direct characterization of photoinduced lattice dynamics in $\mathrm{BaFe}_{2} \mathrm{As}_{2}$," Nat. Commun. 6, 7377.

Gerber, S., et al., 2017, "Femtosecond electron-phonon lock-in by photoemission and x-ray free-electron laser," Science 357, 71.

Ghazaryan, A., T. Graß, M. J. Gullans, P. Ghaemi, and M. Hafezi, 2017, "Light-Induced Fractional Quantum Hall Phases in Graphene," Phys. Rev. Lett. 119, 247403.

Ghimire, S., A. D. Dichiara, E. Sistrunk, P. Agostini, L. F. Dimauro, and D. A. Reis, 2011, "Observation of high-order harmonic generation in a bulk crystal," Nat. Phys. 7, 138.

Ghimire, S., and D. A. Reis, 2019, "High-harmonic generation from solids," Nat. Phys. 15, 10.

Giannetti, C., M. Capone, D. Fausti, M. Fabrizio, F. Parmigiani, and D. Mihailovic, 2016, "Ultrafast optical spectroscopy of strongly correlated materials and high-temperature superconductors: A nonequilibrium approach," Adv. Phys. 65, 58.

Gierz, I., J. C. Petersen, M. Mitrano, C. Cacho, I. C. E. Turcu, E. Springate, A. Stöhr, A. Köhler, U. Starke, and A. Cavalleri, 2013, "Snapshots of non-equilibrium Dirac carrier distributions in graphene," Nat. Mater. 12, 1119.

Gillmeister, K., D. Golež, C.-T. Chiang, N. Bittner, Y. Pavlyukh, J. Berakdar, P. Werner, and W. Widdra, 2020, "Ultrafast coupled charge and spin dynamics in strongly correlated $\mathrm{NiO}$," Nat. Commun. 11, 4095.

Giorgianni, F., T. Cea, C. Vicario, C. P. Hauri, W. K. Withanage, X. $\mathrm{Xi}$, and L. Benfatto, 2019, "Leggett mode controlled by light pulses," Nat. Phys. 15, 341.
Giorgianni, F., B. Wehinger, S. Allenspach, N. Colonna, C. Vicario, P. Puphal, E. Pomjakushina, B. Normand, and C. Rüegg, 2021, "Nonlinear quantum magnetophononics in $\mathrm{SrCu}_{2}\left(\mathrm{BO}_{3}\right)_{2}$," arXiv: 2101.01189.

Giustino, F., et al., 2021, "The 2021 quantum materials roadmap," J. Phys Mater. 3, 042006.

Golež, D, M. Eckstein, and P. Werner, 2019, "Multiband nonequilibrium $G W+$ EDMFT formalism for correlated insulators," Phys. Rev. B 100, 235117.

Görg, F., M. Messer, K. Sandholzer, G. Jotzu, R. Desbuquois, and T. Esslinger, 2018, "Enhancement and sign change of magnetic correlations in a driven quantum many-body system," Nature (London) 553, 481.

Greiter, M., X.-G. Wen, and F. Wilczek, 1991, "Paired Hall State at Half Filling," Phys. Rev. Lett. 66, 3205.

Gross, E. P., 1961, "Structure of a quantized vortex in boson systems," Nuovo Cimento (1955-1965) 20, 454.

Güdde, J., and U. Höfer, 2021, "Ultrafast dynamics of photocurrents in surface states of three-dimensional topological insulators," Phys. Status Solidi B 258, 2000521.

Gulden, T., E. Berg, M.S. Rudner, and N.H. Lindner, 2020, "Exponentially long lifetime of universal quasi-steady states in topological Floquet pumps," SciPost Phys. 9, 15.

Hackl, L., T. Guaita, T. Shi, J. Haegeman, E. Demler, and J. I. Cirac, 2020, "Geometry of variational methods: Dynamics of closed quantum systems," SciPost Phys. 9, 48.

Haight, R., J. A. Silberman, and M. I. Lilie, 1988, "Novel system for picosecond photoemission spectroscopy," Rev. Sci. Instrum. 59, 1941.

Haldane, F. D. M., 1988, "Model for a Quantum Hall Effect without Landau Levels: Condensed-Matter Realization of the 'Parity Anomaly,'” Phys. Rev. Lett. 61, 2015.

Haldar, A., R. Moessner, and A. Das, 2018, "Onset of Floquet thermalization,” Phys. Rev. B 97, 245122.

Harb, M., H. Enquist, A. Jurgilaitis, F. T. Tuyakova, A. N. Obraztsov, and J. Larsson, 2016, "Phonon-phonon interactions in photoexcited graphite studied by ultrafast electron diffraction," Phys. Rev. B 93, 104104.

Haug, H., and A.-P. Jauho, 2008, Quantum Kinetics in Transport and Optics of Semiconductors (Springer, Berlin).

Haupt, K., M. Eichberger, N. Erasmus, A. Rohwer, J. Demsar, K. Rossnagel, and H. Schwoerer, 2016, "Ultrafast Metamorphosis of a Complex Charge-Density Wave," Phys. Rev. Lett. 116, 016402 .

Hejazi, K., J. Liu, and L. Balents, 2019, "Floquet spin and spinorbital Hamiltonians and doublon-holon generations in periodically driven Mott insulators," Phys. Rev. B 99, 205111.

Hellmann, S., et al., 2010, "Ultrafast Melting of a Charge-Density Wave in the Mott Insulator $1 T-\mathrm{TaS}_{2}$," Phys. Rev. Lett. 105, 187401.

Hellmann, S., et al., 2012, "Time-domain classification of chargedensity-wave insulators," Nat. Commun. 3, 1069.

Higuchi, T., C. Heide, K. Ullmann, H. B. Weber, and P. Hommelhoff, 2017, "Light-field-driven currents in graphene," Nature (London) 550, 224.

Ho, W. W., I. Protopopov, and D. A. Abanin, 2018, "Bounds on Energy Absorption and Prethermalization in Quantum Systems with Long-Range Interactions," Phys. Rev. Lett. 120, 200601.

Höfer, U., I. L. Shumay, Ch. Reuß, U. Thomann, W. Wallauer, and Th. Fauster, 1997, "Time-resolved coherent photoelectron spectroscopy of quantized electronic states on metal surfaces," Science 277, 1480.

Hohenberg, P. C., and B. I. Halperin, 1977, "Theory of dynamic critical phenomena,” Rev. Mod. Phys. 49, 435. 
Horstmann, J. G., H. Böckmann, B. Wit, F. Kurtz, G. Storeck, and C. Ropers, 2020, "Coherent control of a surface structural phase transition," Nature (London) 583, 232.

Hu, W., S. Kaiser, D. Nicoletti, C. R. Hunt, I. Gierz, M. C. Hoffmann, M. Le Tacon, T. Loew, B. Keimer, and A. Cavalleri, 2014, "Optically enhanced coherent transport in $\mathrm{YBa}_{2} \mathrm{Cu}_{3} \mathrm{O}_{6.5}$ by ultrafast redistribution of interlayer coupling," Nat. Mater. 13, 705 .

Hübener, H., U. De Giovannini, C. Schäfer, J. Andberger, M. Ruggenthaler, J. Faist, and A. Rubio, 2021, "Engineering quantum materials with chiral optical cavities," Nat. Mater. 20, 438.

Huber, M. A., M. Plankl, M. Eisele, R. E. Marvel, F. Sandner, T. Korn, C. Schüller, R. F. Haglund, Jr., R. Huber, and T. L. Cocker, 2016, "Ultrafast mid-infrared nanoscopy of strained vanadium dioxide nanobeams," Nano Lett. 16, 1421.

Huber, M. A., et al., 2017, "Femtosecond photo-switching of interface polaritons in black phosphorus heterostructures," Nat. Nanotechnol. 12, 207.

Huber, T., et al., 2014, "Coherent Structural Dynamics of a Prototypical Charge-Density-Wave-to-Metal Transition," Phys. Rev. Lett. 113, 026401.

Iadecola, T., T. Neupert, and C. Chamon, 2015, "Occupation of topological Floquet bands in open systems," Phys. Rev. B 91, 235133

Ichikawa, H., et al., 2011, “Transient photoinduced 'hidden' phase in a manganite," Nat. Mater. 10, 101.

Imada, M., A. Fujimori, and Y. Tokura, 1998, "Metal-insulator transitions," Rev. Mod. Phys. 70, 1039.

Ishioka, K., M. Hase, M. Kitajima, L. Wirtz, A. Rubio, and H. Petek, 2008, "Ultrafast electron-phonon decoupling in graphite," Phys. Rev. B 77, 121402(R).

Itin, A. P., and M. I. Katsnelson, 2015, "Effective Hamiltonians for Rapidly Driven Many-Body Lattice Systems: Induced Exchange Interactions and Density-Dependent Hoppings," Phys. Rev. Lett. 115, 075301.

Jager, M. F., C. Ott, P. M. Kraus, C. J. Kaplan, W. Pouse, R. E. Marvel, R. F. Haglund, D. M. Neumark, and S. R. Leone, 2017, "Tracking the insulator-to-metal phase transition in $\mathrm{VO}_{2}$ with fewfemtosecond extreme UV transient absorption spectroscopy," Proc. Natl. Acad. Sci. U.S.A. 114, 9558.

Johannsen, J. C., et al., 2013, "Direct View of Hot Carrier Dynamics in Graphene," Phys. Rev. Lett. 111, 027403.

Johnson, S. L., P. Beaud, C. J. Milne, F. S. Krasniqi, E. S. Zijlstra, M. E. Garcia, M. Kaiser, D. Grolimund, R. Abela, and G. Ingold, 2008, "Nanoscale Depth-Resolved Coherent Femtosecond Motion in Laser-Excited Bismuth," Phys. Rev. Lett. 100, 155501.

Johnson, S. L., et al., 2012, "Femtosecond Dynamics of the Collinear-to-Spiral Antiferromagnetic Phase Transition in CuO," Phys. Rev. Lett. 108, 037203.

Jotzu, G., M. Messer, R. Desbuquois, M. Lebrat, T. Uehlinger, D. Greif, and T. Esslinger, 2014, "Experimental realization of the topological Haldane model with ultracold fermions," Nature (London) 515, 237.

Juraschek, D. M., P. Narang, and N. A. Spaldin, 2020, "Phonomagnetic analogs to opto-magnetic effects," Phys. Rev. Research 2, 043035.

Juraschek, D. M., T. Neuman, J. Flick, and P. Narang, 2021, "Cavity control of nonlinear phononics," Phys. Rev. Research 3, L032046.

Kaindl, R. A., M. Woerner, T. Elsaesser, D. C. Smith, J. F. Ryan, G. A. Farnan, M. P. McCurry, and D. G. Walmsley, 2000, "Ultrafast mid-infrared response of $\mathrm{YBa}_{2} \mathrm{Cu}_{3} \mathrm{O}_{7-\delta}$," Science 287, 470.

Kaiser, S., et al., 2014a, "Optical properties of a vibrationally modulated solid state Mott insulator," Sci. Rep. 4, 3823.
Kaiser, S., et al., 2014b, "Optically induced coherent transport far above $T_{c}$ in underdoped $\mathrm{YBa}_{2} \mathrm{Cu}_{3} \mathrm{O}_{6+\delta}$," Phys. Rev. B 89, 184516.

Kalthoff, M. H., D. M. Kennes, A. J. Millis, and M. A. Sentef, 2021, "Nonequilibrium phase transition in a driven-dissipative quantum antiferromagnet," arXiv:2107.03841.

Kampfrath, T., A. Sell, G. Klatt, A. Pashkin, S. Mährlein, T. Dekorsy, M. Wolf, M. Fiebig, A. Leitenstorfer, and R. Huber, 2011, "Coherent terahertz control of antiferromagnetic spin waves," Nat. Photonics 5, 31.

Kampfrath, T., K. Tanaka, and K. A. Nelson, 2013, "Resonant and nonresonant control over matter and light by intense terahertz transients," Nat. Photonics 7, 680.

Kastl, C., C. Karnetzky, H. Karl, and A. W. Holleitner, 2015, "Ultrafast helicity control of surface currents in topological insulators with near-unity fidelity," Nat. Commun. 6, 6617.

Kealhofer, C., W. Schneider, D. Ehberger, A. Ryabov, F. Krausz, and P. Baum, 2016, "All-optical control and metrology of electron pulses," Science 352, 429.

Keimer, B., and J.E. Moore, 2017, "The physics of quantum materials," Nat. Phys. 13, 1045.

Kemper, A. F., O. Abdurazakov, and J. K. Freericks, 2018, "General Principles for the Nonequilibrium Relaxation of Populations in Quantum Materials," Phys. Rev. X 8, 041009.

Kemper, A. F., M. A. Sentef, B. Moritz, T. P. Devereaux, and J. K. Freericks, 2017, "Review of the theoretical description of timeresolved angle-resolved photoemission spectroscopy in electronphonon mediated superconductors," Ann. Phys. (Berlin) 529, 1600235 .

Kennes, D. M., M. Claassen, M. A. Sentef, and C. Karrasch, 2019, "Light-induced $d$-wave superconductivity through Floquetengineered Fermi surfaces in cuprates," Phys. Rev. B 100, 075115.

Kennes, D. M., M. Claassen, L. Xian, A. Georges, A. J. Millis, J. Hone, C. R. Dean, D. N. Basov, A. N. Pasupathy, and A. Rubio, 2021, "Moiré heterostructures as a condensed-matter quantum simulator," Nat. Phys. 17, 155.

Kennes, D. M., A. de la Torre, A. Ron, D. Hsieh, and A. J. Millis, 2018, "Floquet Engineering in Quantum Chains," Phys. Rev. Lett. 120, 127601.

Kennes, D. M., S. G. Jakobs, C. Karrasch, and V. Meden, 2012, "Renormalization group approach to time-dependent transport through correlated quantum dots," Phys. Rev. B 85, 085113.

Kennes, D. M., N. Müller, M. Pletyukhov, C. Weber, C. Bruder, F. Hassler, J. Klinovaja, D. Loss, and H. Schoeller, 2019, "Chiral onedimensional Floquet topological insulators beyond the rotating wave approximation," Phys. Rev. B 100, 041103.

Kennes, D. M., E. Y. Wilner, D. R. Reichman, and A. J. Millis, 2017, "Transient superconductivity from electronic squeezing of optically pumped phonons," Nat. Phys. 13, 479.

Kibble, T. W. B., 1976, "Topology of cosmic domains and strings," J. Phys. A 9, 1387.

Kim, H. W., et al., 2020, "Towards jitter-free ultrafast electron diffraction technology," Nat. Photonics 14, 245.

Kim, J., X. Hong, C. Jin, S.-F. Shi, C.-Y. S. Chang, M.-H. Chiu, L.-J. $\mathrm{Li}$, and F. Wang, 2014, "Ultrafast generation of pseudo-magnetic field for valley excitons in WSe ${ }_{2}$ monolayers," Science 346, 1205.

Kim, M., Y. Nomura, M. Ferrero, P. Seth, O. Parcollet, and A. Georges, 2016, "Enhancing superconductivity in $\mathrm{A}_{3} \mathrm{C}_{60}$ fullerides," Phys. Rev. B 94, 155152.

Kimel, A. V., A. M. Kalashnikova, A. Pogrebna, and A. K. Zvezdin, 2020, "Fundamentals and perspectives of ultrafast photoferroic recording," Phys. Rep. 852, 1.

Kirilyuk, A., A. V. Kimel, and T. Rasing, 2010, "Ultrafast optical manipulation of magnetic order," Rev. Mod. Phys. 82, 2731. 
Kiryukhin, V., D. Casa, J. P. Hill, B. Keimer, A. Vigliante, Y. Tomioka, and Y. Tokura, 1997, "An x-ray-induced insulator-metal transition in a magnetoresistive manganite," Nature (London) 386, 813.

Kitagawa, T., E. Berg, M. Rudner, and E. Demler, 2010, “Topological characterization of periodically driven quantum systems," Phys. Rev. B 82, 235114.

Kitagawa, T., M. A. Broome, A. Fedrizzi, M. S. Rudner, E. Berg, I. Kassal, A. Aspuru-Guzik, E. Demler, and A. G. White, 2012, "Observation of topologically protected bound states in photonic quantum walks," Nat. Commun. 3, 882.

Kitagawa, T., T. Oka, A. Brataas, L. Fu, and E. Demler, 2011, "Transport properties of nonequilibrium systems under the application of light: Photoinduced quantum Hall insulators without Landau levels," Phys. Rev. B 84, 235108.

Kitamura, S., T. Oka, and H. Aoki, 2017, "Probing and controlling spin chirality in Mott insulators by circularly polarized laser," Phys. Rev. B 96, 014406.

Klöckner, C., C. Karrasch, and D. M. Kennes, 2020, "Nonequilibrium Properties of Berezinskii-Kosterlitz-Thouless Phase Transitions," Phys. Rev. Lett. 125, 147601.

Knap, M., M. Babadi, G. Refael, I. Martin, and E. Demler, 2016, "Dynamical Cooper pairing in nonequilibrium electron-phonon systems," Phys. Rev. B 94, 214504.

Kogar, A., et al., 2020, "Light-induced charge density wave in $\mathrm{LaTe}_{3}$," Nat. Phys. 16, 159.

Komnik, A., and M. Thorwart, 2016, "BCS theory of driven superconductivity,” Eur. Phys. J. B 89, 244.

Konstantinova, T., et al., 2018, "Nonequilibrium electron and lattice dynamics of strongly correlated $\mathrm{Bi}_{2} \mathrm{Sr}_{2} \mathrm{CaCu}_{2} \mathrm{O}_{8+\delta}$ single crystals," Sci. Adv. 4, eaap7427.

Koopmans, B., G. Malinowski, F. Dalla Longa, D. Steiauf, M. Fähnle, T. Roth, M. Cinchetti, and M. Aeschlimann, 2010, "Explaining the paradoxical diversity of ultrafast laser-induced demagnetization,” Nat. Mater. 9, 259.

Koshihara, S., Y. Tokura, T. Mitani, G. Saito, and T. Koda, 1990, "Photoinduced valence instability in the organic molecular compound tetrathiafulvalene- $p$-chloranil (TTF-CA)," Phys. Rev. B 42, 6853.

Kozina, M., et al., 2019, "Terahertz-driven phonon upconversion in $\mathrm{SrTiO}_{3}$," Nat. Phys. 15, 387.

Kubacka, T., et al., 2014, "Large-amplitude spin dynamics driven by a THz pulse in resonance with an electromagnon," Science $\mathbf{3 4 3}$, 1333.

Kundu, A., and B. Seradjeh, 2013, "Transport Signatures of Floquet Majorana Fermions in Driven Topological Superconductors," Phys. Rev. Lett. 111, 136402.

Kung, Y. F., W.-S. Lee, C.-C. Chen, A. F. Kemper, A. P. Sorini, B. Moritz, and T. P. Devereaux, 2013, "Time-dependent charge-order and spin-order recovery in striped systems," Phys. Rev. B 88, 125114.

Kutnyakhov, D., et al., 2020, "Time- and momentum-resolved photoemission studies using time-of-flight momentum microscopy at a free-electron laser," Rev. Sci. Instrum. 91, 013109.

Kuwahara, T., T. Mori, and K. Saito, 2016, "Floquet-Magnus theory and generic transient dynamics in periodically driven many-body quantum systems," Ann. Phys. (Amsterdam) 367, 96.

Laplace, Y., and A. Cavalleri, 2016, "Josephson plasmonics in layered superconductors," Adv. Phys. X 1, 387.

Laulhé, C., et al., 2017, "Ultrafast Formation of a Charge Density Wave State in $1 T-\mathrm{TaS}_{2}$ : Observation at Nanometer Scales Using Time-Resolved X-Ray Diffraction,” Phys. Rev. Lett. 118, 247401 .
Lazarides, A., A. Das, and R. Moessner, 2014, "Equilibrium states of generic quantum systems subject to periodic driving," Phys. Rev. E 90, 012110.

Lazarides, A., A. Das, and R. Moessner, 2015, "Fate of Many-Body Localization under Periodic Driving," Phys. Rev. Lett. 115, 030402.

Lee, C. H., W. W. Ho, B. Yang, J. Gong, and Z. Papić, 2018, "Floquet Mechanism for Non-Abelian Fractional Quantum Hall States," Phys. Rev. Lett. 121, 237401.

Lee, W.S., et al., 2012, "Phase fluctuations and the absence of topological defects in a photo-excited charge-ordered nickelate," Nat. Commun. 3, 838.

Le Guyader, L., T. Chase, A. H. Reid, R. K. Li, D. Svetin, X. Shen, T. Vecchione, X. J. Wang, D. Mihailovic, and H. A. Dürr, 2017, "Stacking order dynamics in the quasi-two-dimensional dichalcogenide $1 T-\mathrm{TaS}_{2}$ probed with $\mathrm{MeV}$ ultrafast electron diffraction," Struct. Dyn. 4, 044020.

Li, J., H. U. R. Strand, P. Werner, and M. Eckstein, 2018, "Theory of photoinduced ultrafast switching to a spin-orbital ordered hidden phase," Nat. Commun. 9, 4581.

Li, T., A. Patz, L. Mouchliadis, J. Yan, T. A. Lograsso, I. E. Perakis, and J. Wang, 2013, "Femtosecond switching of magnetism via strongly correlated spin-charge quantum excitations," Nature (London) 496, 69.

Li, X., T. Qiu, J. Zhang, E. Baldini, J. Lu, A. M. Rappe, and K. A. Nelson, 2019, "Terahertz field-induced ferroelectricity in quantum paraelectric $\mathrm{SrTiO}_{3}$," Science 364, 1079.

Lian, C., S.-J. Zhang, S.-Q. Hu, M.-X. Guan, and S. Meng, 2020, "Ultrafast charge ordering by self-amplified exciton-phonon dynamics in $\mathrm{TiSe}_{2}$, , Nat. Commun. 11, 43.

Ligges, M., et al., 2018, "Ultrafast Doublon Dynamics in Photoexcited $1 T-\mathrm{TaS}_{2}$, , Phys. Rev. Lett. 120, 166401.

Lindner, N. H., G. Refael, and V. Galitski, 2011, "Floquet topological insulator in semiconductor quantum wells," Nat. Phys. 7, 490.

Liu, B., H. Bromberger, A. Cartella, T. Gebert, M. Först, and A. Cavalleri, 2017, "Generation of narrowband, high-intensity, carrier-envelope phase-stable pulses tunable between 4 and 18 THz," Opt. Lett. 42, 129.

Liu, J., K. Hejazi, and L. Balents, 2018, "Floquet Engineering of Multiorbital Mott Insulators: Applications to Orthorhombic Titanates," Phys. Rev. Lett. 121, 107201.

Liu, M., et al., 2012, "Terahertz-field-induced insulator-to-metal transition in vanadium dioxide metamaterial,"Nature (London) $\mathbf{4 8 7}$, 345.

Lovinger, D. J., M. Brahlek, P. Kissin, D. M. Kennes, A. J. Millis, R. Engel-Herbert, and R. D. Averitt, 2020, "Influence of spin and orbital fluctuations on Mott-Hubbard exciton dynamics in $\mathrm{LaVO}_{3}$ thin films," Phys. Rev. B 102, 115143.

Lucchini, M., S. A. Sato, A. Ludwig, J. Herrmann, M. Volkov, L. Kasmi, Y. Shinohara, K. Yabana, L. Gallmann, and U. Keller, 2016, "Attosecond dynamical Franz-Keldysh effect in polycrystalline diamond," Science 353, 916.

Luitz, D. J., R. Moessner, S. L. Sondhi, and V. Khemani, 2020, "Prethermalization without Temperature," Phys. Rev. X 10, 021046.

Lv, B., T. Qian, and H. Ding, 2019, "Angle-resolved photoemission spectroscopy and its application to topological materials," Nat. Rev. Phys. 1, 609.

Ma, Q., et al., 2016, "Tuning ultrafast electron thermalization pathways in a van der Waals heterostructure," Nat. Phys. 12, 455.

Machado, F., D. V. Else, G. D. Kahanamoku-Meyer, C. Nayak, and N. Y. Yao, 2020, "Long-Range Prethermal Phases of Nonequilibrium Matter,” Phys. Rev. X 10, 011043. 
Machado, F., G. D. Kahanamoku-Meyer, D. V. Else, C. Nayak, and N. Y. Yao, 2019, "Exponentially slow heating in short and long-range interacting Floquet systems," Phys. Rev. Research 1, 033202.

Maczewsky, L. J., J. M. Zeuner, S. Nolte, and A. Szameit, 2017, "Observation of photonic anomalous Floquet topological insulators," Nat. Commun. 8, 13756.

Madéo, J., et al., 2020, "Directly visualizing the momentumforbidden dark excitons and their dynamics in atomically thin semiconductors," Science 370, 1199.

Mahmood, F., Z. Alpichshev, Y.-H. Lee, J. Kong, and N. Gedik, 2018, "Observation of exciton-exciton interaction mediated valley depolarization in monolayer $\mathrm{MoSe}_{2}$," Nano Lett. 18, 223.

Mahmood, F., C.-K. Chan, Z. Alpichshev, D. Gardner, Y. Lee, P. A. Lee, and N. Gedik, 2016, "Selective scattering between FloquetBloch and Volkov states in a topological insulator," Nat. Phys. 12, 306.

Mahmood, F., D. Chaudhuri, S. Gopalakrishnan, R. Nandkishore, and N. P. Armitage, 2021, "Observation of a marginal Fermi glass using THz 2D coherent spectroscopy," Nat. Phys. 17, 627.

Mankowsky, R., M. Först, and A. Cavalleri, 2016, "Non-equilibrium control of complex solids by nonlinear phononics," Rep. Prog. Phys. 79, 064503.

Mankowsky, R., M. Först, T. Loew, J. Porras, B. Keimer, and A. Cavalleri, 2015, "Coherent modulation of the $\mathrm{YBa}_{2} \mathrm{Cu}_{3} \mathrm{O}_{6+x}$ atomic structure by displacive stimulated ionic Raman scattering," Phys. Rev. B 91, 094308.

Mankowsky, R., A. von Hoegen, M. Först, and A. Cavalleri, 2017, "Ultrafast Reversal of the Ferroelectric Polarization," Phys. Rev. Lett. 118, 197601.

Mankowsky, R., et al., 2014, "Nonlinear lattice dynamics as a basis for enhanced superconductivity in $\mathrm{YBa}_{2} \mathrm{Cu}_{3} \mathrm{O}_{6.5}$," Nature (London) 516, 71.

Mannebach, E. M., et al., 2015, "Dynamic structural response and deformations of monolayer $\mathrm{MoS}_{2}$ visualized by femtosecond electron diffraction," Nano Lett. 15, 6889.

Mardegan, J. R. L., et al., 2021, "Ultrafast electron localization in the $\mathrm{EuNi}_{2}\left(\mathrm{Si}_{0.21} \mathrm{Ge}_{0.79}\right)_{2}$ correlated metal," Phys. Rev. Research 3, 033211.

Marques, M. A. L., N. T. Maitra, F. M. S. Nogueira, E. K. U. Gross, and A. Rubio, Eds. 2012, Fundamentals of Time-Dependent Density Functional Theory, Lecture Notes in Physics Vol. 837 (Springer-Verlag, Berlin).

Massicotte, M., P. Schmidt, F. Vialla, K. G. Schädler, A. ReserbatPlantey, K. Watanabe, T. Taniguchi, K. J. Tielrooij, and F. H. L. Koppens, 2016, "Picosecond photoresponse in van der Waals heterostructures," Nat. Nanotechnol. 11, 42.

Mathey, S., and S. Diehl, 2019, "Absence of Criticality in the Phase Transitions of Open Floquet Systems," Phys. Rev. Lett. 122, 110602.

Matsunaga, R., Y. I. Hamada, K. Makise, Y. Uzawa, H. Terai, Z. Wang, and R. Shimano, 2013, "Higgs Amplitude Mode in the BCS Superconductors $\mathrm{Nb}_{1-x} \mathrm{Ti}_{x} \mathrm{~N}$ Induced by Terahertz Pulse Excitation," Phys. Rev. Lett. 111, 057002.

Matsunaga, R., N. Tsuji, H. Fujita, A. Sugioka, K. Makise, Y. Uzawa, H. Terai, Z. Wang, H. Aoki, and R. Shimano, 2014, "Light-induced collective pseudospin precession resonating with Higgs mode in a superconductor," Science 345, 1145.

Mazza, G., and A. Georges, 2017, "Nonequilibrium superconductivity in driven alkali-doped fullerides," Phys. Rev. B 96, 064515.

Mazzone, D. G., et al., 2021, "Laser-induced transient magnons in $\mathrm{Sr}_{3} \mathrm{Ir}_{2} \mathrm{O}_{7}$ throughout the Brillouin zone," Proc. Natl. Acad. Sci. U.S.A. 118, e2103696118.
McIver, J. W., B. Schulte, F. U. Stein, T. Matsuyama, G. Jotzu, G. Meier, and A. Cavalleri, 2020, "Light-induced anomalous Hall effect in graphene," Nat. Phys. 16, 38.

Mendoza-Arenas, J. J., J. F. Gomez-Ruiz, M. Eckstein, D. Jaksch, and S. R. Clark, 2017, "Ultra-fast control of magnetic relaxation in a periodically driven Hubbard model," Ann. Phys. (Berlin) 529, 1700024

Mentink, J. H., K. Balzer, and M. Eckstein, 2015, "Ultrafast and reversible control of the exchange interaction in Mott insulators," Nat. Commun. 6, 6708.

Mertelj, T., P. Kusar, V. V. Kabanov, P. Giraldo-Gallo, I. R. Fisher, and D. Mihailovic, 2013, "Incoherent Topological Defect Recombination Dynamics in $\mathrm{TbTe}_{3}$," Phys. Rev. Lett. 110, 156401.

Mihailovic, D., 2019, "The importance of topological defects in photoexcited phase transitions including memory applications," Appl. Sci. 9, 890.

Mikami, T., S. Kitamura, K. Yasuda, N. Tsuji, T. Oka, and H. Aoki, 2016, "Brillouin-Wigner theory for high-frequency expansion in periodically driven systems: Application to Floquet topological insulators," Phys. Rev. B 93, 144307.

Mitra, A., S. Takei, Y. B. Kim, and A. J. Millis, 2006, "Nonequilibrium Quantum Criticality in Open Electronic Systems," Phys. Rev. Lett. 97, 236808.

Mitrano, M., and Y. Wang, 2020, "Probing light-driven quantum materials with ultrafast resonant inelastic X-ray scattering," Commun. Phys. 3, 184.

Mitrano, M., et al., 2014, "Pressure-Dependent Relaxation in the Photoexcited Mott Insulator ET-F $\mathrm{F}_{2}$ TCNQ: Influence of Hopping and Correlations on Quasiparticle Recombination Rates," Phys. Rev. Lett. 112, 117801.

Mitrano, M., et al., 2016, "Possible light-induced superconductivity in $\mathrm{K}_{3} \mathrm{C}_{60}$ at high temperature," Nature (London) 530, 461.

Mitrano, M., et al., 2019, "Ultrafast time-resolved x-ray scattering reveals diffusive charge order dynamics in $\mathrm{La}_{2-x} \mathrm{Ba}_{x} \mathrm{CuO}_{4}$," Sci. Adv. 5, eaax3346.

Miyake, T., F. Aryasetiawan, and M. Imada, 2009, “Ab initio procedure for constructing effective models of correlated materials with entangled band structure," Phys. Rev. B 80, 155134.

Miyano, K., T. Tanaka, Y. Tomioka, and Y. Tokura, 1997, "Photoinduced Insulator-to-Metal Transition in a Perovskite Manganite," Phys. Rev. Lett. 78, 4257.

Moore, G., and N. Read, 1991, "Nonabelions in the fractional quantum Hall effect," Nucl. Phys. B360, 362.

Mori, T., T. N. Ikeda, E. Kaminishi, and M. Ueda, 2018, “Thermalization and prethermalization in isolated quantum systems: A theoretical overview," J. Phys. B 51, 112001.

Mori, T., T. Kuwahara, and K. Saito, 2016, "Rigorous Bound on Energy Absorption and Generic Relaxation in Periodically Driven Quantum Systems,” Phys. Rev. Lett. 116, 120401.

Morrison, V. R., R. P. Chatelain, K. L. Tiwari, A. Hendaoui, A. Bruhács, M. Chaker, and B. J. Siwick, 2014, "A photoinduced metal-like phase of monoclinic $\mathrm{VO}_{2}$ revealed by ultrafast electron diffraction," Science 346, 445.

Mraz, A., et al., 2021, "Energy efficient manipulation of topologically protected states in non-volatile ultrafast charge configuration memory devices," arXiv:2103.04622.

Mücke, O. D., T. Tritschler, M. Wegener, U. Morgner, and F. X. Kärtner, 2001, "Signatures of Carrier-Wave Rabi Flopping in GaAs,” Phys. Rev. Lett. 87, 057401.

Murakami, Y., N. Tsuji, M. Eckstein, and P. Werner, 2017, "Nonequilibrium steady states and transient dynamics of conventional superconductors under phonon driving," Phys. Rev. B 96, 045125. 
Murakami, Y., P. Werner, N. Tsuji, and H. Aoki, 2015, "Interaction quench in the Holstein model: Thermalization crossover from electron- to phonon-dominated relaxation," Phys. Rev. B 91, 045128

Na, M. X., et al., 2019, "Direct determination of mode-projected electron-phonon coupling in the time domain," Science $\mathbf{3 6 6}$, 1231.

Na, M. X., et al., 2020, "Establishing nonthermal regimes in pumpprobe electron relaxation dynamics," Phys. Rev. B 102, 184307.

Narang, P., C. A. C. Garcia, and C. Felser, 2021, "The topology of electronic band structures," Nat. Mater. 20, 293.

Naseska, M., P. Sutar, Y. Vaskivskyi, I. Vaskivskyi, D. Vengust, D. Svetin, V. V. Kabanov, D. Mihailovic, and T. Mertelj, 2021, "Firstorder kinetics bottleneck during photoinduced ultrafast insulatormetal transition in 3D orbitally-driven Peierls insulator $\mathrm{CuIr}_{2} \mathrm{~S}_{4}$," New J. Phys. 23, 053023.

Nasu, K., H. Ping, and H. Mizouchi, 2001, "Photoinduced structural phase transitions and their dynamics," J. Phys. Condens. Matter 13, R693.

Nava, A., C. Giannetti, A. Georges, E. Tosatti, and M. Fabrizio, 2018, "Cooling quasiparticles in $\mathrm{A}_{3} \mathrm{C}_{60}$ fullerides by excitonic midinfrared absorption," Nat. Phys. 14, 154.

Nayak, C., S. H. Simon, A. Stern, M. Freedman, and S. Das Sarma, 2008, "Non-Abelian anyons and topological quantum computation," Rev. Mod. Phys. 80, 1083.

Němec, P, M. Fiebig, T. Kampfrath, and A. V. Kimel, 2018, "Antiferromagnetic opto-spintronics," Nat. Phys. 14, 229.

Ni, G.X., et al., 2016, "Ultrafast optical switching of infrared plasmon polaritons in high-mobility graphene," Nat. Photonics 10, 244.

Nicholson, C. W., A. Lücke, W. G. Schmidt, M. Puppin, L. Rettig, R. Ernstorfer, and M. Wolf, 2018, "Beyond the molecular movie: Dynamics of bands and bonds during a photoinduced phase transition," Science 362, 821.

Nicoletti, D., E. Casandruc, Y. Laplace, V. Khanna, C. R. Hunt, S. Kaiser, S. S. Dhesi, G. D. Gu, J. P. Hill, and A. Cavalleri, 2014, "Optically induced superconductivity in striped $\mathrm{La}_{2-x} \mathrm{Ba}_{x} \mathrm{CuO}_{4}$ by polarization-selective excitation in the near infrared," Phys. Rev. B 90, 100503.

Nicoletti, D., D. Fu, O. Mehio, S. Moore, A. S. Disa, G. D. Gu, and A. Cavalleri, 2018, "Magnetic-Field Tuning of Light-Induced Superconductivity in Striped $\mathrm{La}_{2-x} \mathrm{Ba}_{x} \mathrm{CuO}_{4}$," Phys. Rev. Lett. 121, 267003.

Nova, T. F., A. Cartella, A. Cantaluppi, M. Först, D. Bossini, R. V. Mikhaylovskiy, A. V. Kimel, R. Merlin, and A. Cavalleri, 2017, "An effective magnetic field from optically driven phonons," Nat. Phys. 13, 132.

Nova, T.F., A.S. Disa, M. Fechner, and A. Cavalleri, 2019, "Metastable ferroelectricity in optically strained $\mathrm{SrTiO}_{3}$," Science 364, 1075.

Nunes, Jr., G., and M. R. Freeman, 1993, "Picosecond resolution in scanning tunneling microscopy," Science 262, 1029.

Nuske, M., L. Broers, B. Schulte, G. Jotzu, S. A. Sato, A. Cavalleri, A. Rubio, J. W. McIver, and L. Mathey, 2020, "Floquet dynamics in light-driven solids," Phys. Rev. Research 2, 043408.

Ogawa, S., H. Nagano, H. Petek, and A. P. Heberle, 1997, "Optical Dephasing in $\mathrm{Cu}(111)$ Measured by Interferometric Two-Photon Time-Resolved Photoemission," Phys. Rev. Lett. 78, 1339.

Oka, T., and H. Aoki, 2009, "Photovoltaic Hall effect in graphene," Phys. Rev. B 79, 081406.

Oka, T., and S. Kitamura, 2019, "Floquet engineering of quantum materials," Annu. Rev. Condens. Matter Phys. 10, 387.
Okamoto, J.-i., A. Cavalleri, and L. Mathey, 2016, "Theory of Enhanced Interlayer Tunneling in Optically Driven High- $T_{c}$ Superconductors," Phys. Rev. Lett. 117, 227001.

Ono, A., and S. Ishihara, 2019, "Nonequilibrium susceptibility in photoinduced Floquet states," Phys. Rev. B 100, 075127.

Orenstein, J., 2012, "Ultrafast spectroscopy of quantum materials," Phys. Today 65, No. 9, 44.

Orús, R., 2014, "A practical introduction to tensor networks: Matrix product states and projected entangled pair states," Ann. Phys. (Amsterdam) 349, 117.

Otto, M. R., L.P. René de Cotret, D. A. Valverde-Chavez, K. L. Tiwari, N. Émond, M. Chaker, D. G. Cooke, and B. J. Siwick, 2019, "How optical excitation controls the structure and properties of vanadium dioxide," Proc. Natl. Acad. Sci. U.S.A. 116, 450.

Paeckel, S., T. Köhler, A. Swoboda, S. R. Manmana, U. Schollwöck, and C. Hubig, 2019, "Time-evolution methods for matrix-product states," Ann. Phys. (Amsterdam) 411, 167998.

Parchenko, S., et al., 2020, "Orbital dynamics during an ultrafast insulator to metal transition," Phys. Rev. Research 2, 023110.

Parham, S., H. Li, T. J. Nummy, J. A. Waugh, X. Q. Zhou, J. Griffith, J. Schneeloch, R. D. Zhong, G. D. Gu, and D. S. Dessau, 2017, "Ultrafast Gap Dynamics and Electronic Interactions in a Photoexcited Cuprate Superconductor," Phys. Rev. X 7, 041013.

Paris, E., et al., 2021, "Probing the interplay between lattice dynamics and short-range magnetic correlations in $\mathrm{CuGeO}_{3}$ with femtosecond RIXS," npj Quantum Mater. 6, 51.

Patz, A., T. Li, S. Ran, R. M. Fernandes, J. Schmalian, S. L. Bud'ko, P. C. Canfield, I. E. Perakis, and J. Wang, 2014, "Ultrafast observation of critical nematic fluctuations and giant magnetoelastic coupling in iron pnictides," Nat. Commun. 5, 3229.

Perfetti, L., P. A. Loukakos, M. Lisowski, U. Bovensiepen, H. Berger, S. Biermann, P. S. Cornaglia, A. Georges, and M. Wolf, 2006, "Time Evolution of the Electronic Structure of $1 T-\mathrm{TaS}_{2}$ through the Insulator-Metal Transition," Phys. Rev. Lett. 97, 067402.

Peronaci, F., M. Schiro, and O. Parcollet, 2018, "Resonant Thermalization of Periodically Driven Strongly Correlated Electrons," Phys. Rev. Lett. 120, 197601.

Petek, H., and S. Ogawa, 1997, "Femtosecond time-resolved twophoton photoemission studies of electron dynamics in metals," Prog. Surf. Sci. 56, 239.

Pitaevskii, L. P., 1961, "Vortex lines in an imperfect Bose gas," Sov. Phys. JETP 13, 451, http://www.jetp.ras.ru/cgi-bin/e/index/e/13/2/ p451?a=list.

Polkovnikov, A., K. Sengupta, A. Silva, and M. Vengalattore, 2011, "Nonequilibrium dynamics of closed interacting quantum systems," Rev. Mod. Phys. 83, 863.

Ponte, P., Z. Papić, F. Huveneers, and D. A. Abanin, 2015, "ManyBody Localization in Periodically Driven Systems," Phys. Rev. Lett. 114, 140401.

Porer, M., U. Leierseder, J. M. Ménard, H. Dachraoui, L. Mouchliadis, I. E. Perakis, U. Heinzmann, J. Demsar, K. Rossnagel, and R. Huber, 2014, "Non-thermal separation of electronic and structural orders in a persisting charge density wave," Nat. Mater. 13, 857.

Qi, F., et al., 2020, "Breaking 50 Femtosecond Resolution Barrier in $\mathrm{MeV}$ Ultrafast Electron Diffraction with a Double Bend Achromat Compressor," Phys. Rev. Lett. 124, 134803.

Qi, T., Y.-H. Shin, K.-L. Yeh, K. A. Nelson, and A. M. Rappe, 2009, "Collective Coherent Control: Synchronization of Polarization in Ferroelectric $\mathrm{PbTiO}_{3}$ by Shaped THz Fields," Phys. Rev. Lett. 102, 247603. 
Raines, Z. M., V. Stanev, and V. M. Galitski, 2015, "Enhancement of superconductivity via periodic modulation in a three-dimensional model of cuprates," Phys. Rev. B 91, 184506.

Rajasekaran, S., E. Casandruc, Y. Laplace, D. Nicoletti, G. D. Gu, S. R. Clark, D. Jaksch, and A. Cavalleri, 2016, "Parametric amplification of a superconducting plasma wave," Nat. Phys. 12, 1012.

Read, N., and E. Rezayi, 1999, "Beyond paired quantum Hall states: Parafermions and incompressible states in the first excited Landau level," Phys. Rev. B 59, 8084.

Rechtsman, M. C., J. M. Zeuner, Y. Plotnik, Y. Lumer, D. Podolsky, F. Dreisow, S. Nolte, M. Segev, and A. Szameit, 2013, "Photonic Floquet topological insulators," Nature (London) 496, 196.

Reimann, J., et al., 2018, "Subcycle observation of lightwave-driven Dirac currents in a topological surface band," Nature (London) 562, 396.

Reimann, K., 2007, "Table-top sources of ultrashort THz pulses," Rep. Prog. Phys. 70, 1597.

René de Cotret, L. P., J.-H. Pöhls, M. J. Stern, M. R. Otto, M. Sutton, and B. J. Siwick, 2019, "Time- and momentum-resolved phonon population dynamics with ultrafast electron diffuse scattering," Phys. Rev. B 100, 214115.

Rettig, L., R. Cortés, J.-H. Chu, I. R. Fisher, F. Schmitt, R. G. Moore, Z.-X. Shen, P. S. Kirchmann, M. Wolf, and U. Bovensiepen, 2016, "Persistent order due to transiently enhanced nesting in an electronically excited charge density wave," Nat. Commun. 7, 10459.

Rettig, L., et al., 2015, "Ultrafast Structural Dynamics of the FePnictide Parent Compound $\mathrm{BaFe}_{2} \mathrm{As}_{2}$," Phys. Rev. Lett. 114, 067402.

Reutzel, M., A. Li, and H. Petek, 2019, "Coherent Two-Dimensional Multiphoton Photoelectron Spectroscopy of Metal Surfaces," Phys. Rev. X 9, 011044.

Reutzel, M., A. Li, Z. Wang, and H. Petek, 2020, "Coherent multidimensional photoelectron spectroscopy of ultrafast quasiparticle dressing by light," Nat. Commun. 11, 2230.

Rini, M., R. Tobey, N. Dean, J. Itatani, Y. Tomioka, Y. Tokura, R. W. Schoenlein, and A. Cavalleri, 2007, "Control of the electronic phase of a manganite by mode-selective vibrational excitation," Nature (London) 449, 72.

Rischel, C., A. Rousse, I. Uschmann, P.-A. Albouy, J.-P. Geindre, P. Audebert, J.-C. Gauthier, E. Fröster, J.-L. Martin, and A. Antonetti, 1997, "Femtosecond time-resolved x-ray diffraction from laserheated organic films," Nature (London) 390, 490.

Rodriguez-Vega, M., M. Vogl, and G. A. Fiete, 2021, "Moiré-Floquet engineering of quantum materials: A review," Ann. Phys. (Amsterdam) (in press), https://doi.org/10.1016/j.aop.2021.168434.

Rohwer, T., et al., 2011, "Collapse of long-range charge order tracked by time-resolved photoemission at high momenta," Nature (London) 471, 490.

Rossi, F., and T. Kuhn, 2002, "Theory of ultrafast phenomena in photoexcited semiconductors," Rev. Mod. Phys. 74, 895.

Rovny, J., R. L. Blum, and S.E. Barrett, 2018, "Observation of Discrete-Time-Crystal Signatures in an Ordered Dipolar ManyBody System,” Phys. Rev. Lett. 120, 180603.

Rudner, M. S., and N. H. Lindner, 2020, "Band structure engineering and non-equilibrium dynamics in Floquet topological insulators," Nat. Rev. Phys. 2, 229.

Rudner, M. S., N.H. Lindner, E. Berg, and M. Levin, 2013, "Anomalous Edge States and the Bulk-Edge Correspondence for Periodically Driven Two-Dimensional Systems," Phys. Rev. X 3, 031005.

Ruggenthaler, M., N. Tancogne-Dejean, J. Flick, H. Appel, and A. Rubio, 2018, "From a quantum-electrodynamical light-matter description to novel spectroscopies," Nat. Rev. Chem. 2, 0118.
Runge, E., and E. K. U. Gross, 1984, "Density-Functional Theory for Time-Dependent Systems," Phys. Rev. Lett. 52, 997.

Sahoo, S., I. Schneider, and S. Eggert, 2019, "Periodically driven many-body systems: A Floquet density matrix renormalization group study," arXiv:1906.00004.

Sala, V. G., S. Dal Conte, T. A. Miller, D. Viola, E. Luppi, V. Véniard, G. Cerullo, and S. Wall, 2016, "Resonant optical control of the structural distortions that drive ultrafast demagnetization in $\mathrm{Cr}_{2} \mathrm{O}_{3}$," Phys. Rev. B 94, 014430.

Sandri, M., and M. Fabrizio, 2013, "Nonequilibrium dynamics in the antiferromagnetic Hubbard model," Phys. Rev. B 88, 165113

Sato, S. A., et al., 2019, "Microscopic theory for the light-induced anomalous Hall effect in graphene," Phys. Rev. B 99, 214302.

Schäfer, W., and M. Wegener, 2002, Semiconductor Optics and Transport Phenomena (Springer, Berlin).

Schiffrin, A., et al., 2013, "Optical-field-induced current in dielectrics," Nature (London) 493, 70.

Schiró, M., and M. Fabrizio, 2010, "Time-Dependent Mean Field Theory for Quench Dynamics in Correlated Electron Systems," Phys. Rev. Lett. 105, 076401.

Schlauderer, S., C. Lange, S. Baierl, T. Ebnet, C. P. Schmid, D. C. Valovcin, A. K. Zvezdin, A. V. Kimel, R. V. Mikhaylovskiy, and R. Huber, 2019, "Temporal and spectral fingerprints of ultrafast allcoherent spin switching," Nature (London) 569, 383.

Schmitt, F., et al., 2008, "Transient electronic structure and melting of a charge density wave in $\mathrm{TbTe}_{3}$," Science 321, 1649.

Schoenlein, R. W., S. Chattopadhyay, H. H. W. Chong, T. E. Glover, P. A. Heimann, C. V. Shank, A. A. Zholents, and M. S. Zolotorev, 2000, "Generation of femtosecond pulses of synchrotron radiation," Science 287, 2237.

Schoenlein, R. W., W. P. Leemans, A. H. Chin, P. Volfbeyn, T. E. Glover, P. Balling, M. Zolotorev, K.-J. Kim, S. Chattopadhyay, and C. V. Shank, 1996, "Femtosecond X-ray pulses at $0.4 \AA$ generated by $90^{\circ}$ Thomson scattering: A tool for probing the structural dynamics of materials," Science 274, 236.

Schoenlein, R. W., W. Z. Lin, J. G. Fujimoto, and G. L. Eesley, 1987, "Femtosecond Studies of Nonequilibrium Electronic Processes in Metals," Phys. Rev. Lett. 58, 1680.

Schollwöck, U., 2011, "The density-matrix renormalization group in the age of matrix product states," Ann. Phys. (Amsterdam) 326, 96.

Schubert, O., et al., 2014, "Sub-cycle control of terahertz highharmonic generation by dynamical Bloch oscillations," Nat. Photonics 8, 119 .

Schüler, M., Y. Murakami, and P. Werner, 2018, "Nonthermal switching of charge order: Dynamical slowing down and optimal control,” Phys. Rev. B 97, 155136.

Schultze, M., et al., 2013, "Controlling dielectrics with the electric field of light," Nature (London) 493, 75.

Scully, M. O., and M. S. Zubairy, 1997, "Atom-field interactionSemiclassical theory," in Quantum Optics (Cambridge University Press, Cambridge, England).

Seaberg, M. H., et al., 2017, "Nanosecond X-Ray Photon Correlation Spectroscopy on Magnetic Skyrmions," Phys. Rev. Lett. 119, 067403.

Seddon, E. A., et al., 2017, "Short-wavelength free-electron laser sources and science: A review," Rep. Prog. Phys. 80, 115901.

Seetharam, K. I., C.-E. Bardyn, N. H. Lindner, M. S. Rudner, and G. Refael, 2015, "Controlled Population of Floquet-Bloch States via Coupling to Bose and Fermi Baths," Phys. Rev. X 5, 041050.

Seetharam, K. I., C.-E. Bardyn, N. H. Lindner, M. S. Rudner, and G. Refael, 2019, "Steady states of interacting Floquet insulators," Phys. Rev. B 99, 014307. 
Segre, G. P., N. Gedik, J. Orenstein, D. A. Bonn, R. Liang, and W. N. Hardy, 2002, "Photoinduced Changes of Reflectivity in Single Crystals of $\mathrm{YBa}_{2} \mathrm{Cu}_{3} \mathrm{O}_{6.5}$ (Ortho II)," Phys. Rev. Lett. 88, 137001.

Seibold, G., and J. Lorenzana, 2001, "Time-Dependent Gutzwiller Approximation for the Hubbard Model," Phys. Rev. Lett. 86, 2605.

Sentef, M. A., 2017, "Light-enhanced electron-phonon coupling from nonlinear electron-phonon coupling," Phys. Rev. B 95, 205111.

Sentef, M. A., M. Claassen, A. F. Kemper, B. Moritz, T. Oka, J. K. Freericks, and T. P. Devereaux, 2015, "Theory of Floquet band formation and local pseudospin textures in pump-probe photoemission of graphene," Nat. Commun. 6, 7047.

Sentef, M. A., A. F. Kemper, A. Georges, and C. Kollath, 2016, "Theory of light-enhanced phonon-mediated superconductivity," Phys. Rev. B 93, 144506.

Sentef, M. A., J. Li, F. Künzel, and M. Eckstein, 2020, “Quantum to classical crossover of Floquet engineering in correlated quantum systems," Phys. Rev. Research 2, 033033.

Sentef, M. A., A. Tokuno, A. Georges, and C. Kollath, 2017, "Theory of Laser-Controlled Competing Superconducting and Charge Orders," Phys. Rev. Lett. 118, 087002.

Shi, T., E. Demler, and J. I. Cirac, 2018, "Variational study of fermionic and bosonic systems with non-Gaussian states: Theory and applications," Ann. Phys. (Amsterdam) 390, 245.

Shimojima, T., Y. Suzuki, A. Nakamura, N. Mitsuishi, S. Kasahara, T. Shibauchi, Y. Matsuda, Y. Ishida, S. Shin, and K. Ishizaka, 2019, "Ultrafast nematic-orbital excitation in FeSe," Nat. Commun. 10, 1946.

Sie, E. J., J. W. McIver, Y.-H. Lee, L. Fu, J. Kong, and N. Gedik, 2015, "Valley-selective optical Stark effect in monolayer $\mathrm{WS}_{2}$," Nat. Mater. 14, 290.

Sie, E. J., et al., 2019, "An ultrafast symmetry switch in a Weyl semimetal," Nature (London) 565, 61.

Sieberer, L. M., M. Buchhold, and S. Diehl, 2016, "Keldysh field theory for driven open quantum systems," Rep. Prog. Phys. 79, 096001.

Siegrist, F., et al., 2019, "Light-wave dynamic control of magnetism," Nature (London) 571, 240.

Singh, K., et al., 2019, "Quantifying and Controlling Prethermal Nonergodicity in Interacting Floquet Matter," Phys. Rev. X 9, 041021 .

Singla, R., et al., 2015, "THz-Frequency Modulation of the Hubbard $U$ in an Organic Mott Insulator,' Phys. Rev. Lett. 115, 187401.

Siwick, B. J., J. R. Dwyer, R. E. Jordan, and R. J. D. Miller, 2003, "An atomic-level view of melting using femtosecond electron diffraction," Science 302, 1382.

Smallwood, C. L., J. P. Hinton, C. Jozwiak, W. Zhang, J. D. Koralek, H. Eisaki, D.-H. Lee, J. Orenstein, and A. Lanzara, 2012, "Tracking Cooper pairs in a cuprate superconductor by ultrafast angleresolved photoemission," Science 336, 1137.

Smallwood, C. L., R. A. Kaindl, and A. Lanzara, 2016, "Ultrafast angle-resolved photoemission spectroscopy of quantum materials," Europhys. Lett. 115, 27001.

Sobota, J. A., Y. He, and Z.-X. Shen, 2021, "Angle-resolved photoemission studies of quantum materials," Rev. Mod. Phys. 93, 025006.

Sobota, J. A., S. Yang, J. G. Analytis, Y. L. Chen, I. R. Fisher, P. S. Kirchmann, and Z.-X. Shen, 2012, "Ultrafast Optical Excitation of a Persistent Surface-State Population in the Topological Insulator $\mathrm{Bi}_{2} \mathrm{Se}_{3}$," Phys. Rev. Lett. 108, 117403.

Sobota, J. A., et al., 2013, "Direct Optical Coupling to an Unoccupied Dirac Surface State in the Topological Insulator $\mathrm{Bi}_{2} \mathrm{Se}_{3}$," Phys. Rev. Lett. 111, 136802.
Spaldin, N. A., 2020, "Multiferroics beyond electric-field control of magnetism," Proc. R. Soc. A 476, 20190542.

Sriram, A., and M. Claassen, 2021, "Light-induced control of magnetic phases in Kitaev quantum magnets," arXiv:2105.01062.

Stamm, C., et al., 2007, "Femtosecond modification of electron localization and transfer of angular momentum in nickel," Nat. Mater. 6, 740.

Stefanucci, G., and R. van Leeuwen, 2013, Nonequilibrium ManyBody Theory of Quantum Systems: A Modern Introduction (Cambridge University Press, Cambridge, England).

Steinmeyer, G., D. H. Sutter, L. Gallmann, N. Matuschek, and U. Keller, 1999, "Frontiers in ultrashort pulse generation: Pushing the limits in linear and nonlinear optics," Science 286, 1507.

Stern, M. J., L. P. René de Cotret, M. R. Otto, R. P. Chatelain, J.-P. Boisvert, M. Sutton, and B. J. Siwick, 2018, "Mapping momentumdependent electron-phonon coupling and nonequilibrium phonon dynamics with ultrafast electron diffuse scattering," Phys. Rev. B 97, 165416.

Stoica, V. A., et al., 2019, "Optical creation of a supercrystal with three-dimensional nanoscale periodicity," Nat. Mater. 18, 377.

Stojchevska, L., I. Vaskivskyi, T. Mertelj, P. Kusar, D. Svetin, S. Brazovskii, and D. Mihailovic, 2014, "Ultrafast switching to a stable hidden quantum state in an electronic crystal," Science 344, 177.

Stormer, H. L., D. C. Tsui, and A. C. Gossard, 1999, "The fractional quantum Hall effect," Rev. Mod. Phys. 71, S298.

Stupakiewicz, A., C. S. Davies, K. Szerenos, D. Afanasiev, K. S. Rabinovich, A. V. Boris, A. Caviglia, A. V. Kimel, and A. Kirilyuk, 2021, "Ultrafast phononic switching of magnetization," Nat. Phys. 17, 489.

Subedi, A., A. Cavalleri, and A. Georges, 2014, "Theory of nonlinear phononics for coherent light control of solids," Phys. Rev. B 89, 220301.

Sun, D., G. Aivazian, A. M. Jones, J. S. Ross, W. Yao, D. Cobden, and X. Xu, 2012, "Ultrafast hot-carrier-dominated photocurrent in graphene," Nat. Nanotechnol. 7, 114.

Sun, Z., and A. J. Millis, 2020, "Transient Trapping into Metastable States in Systems with Competing Orders," Phys. Rev. X 10, 021028.

Takubo, N., Y. Ogimoto, M. Nakamura, H. Tamaru, M. Izumi, and K. Miyano, 2005, "Persistent and Reversible All-Optical Phase Control in a Manganite Thin Film," Phys. Rev. Lett. 95, 017404.

Tancogne-Dejean, N., M. A. Sentef, and A. Rubio, 2018, "Ultrafast Modification of Hubbard $U$ in a Strongly Correlated Material: Ab Initio High-Harmonic Generation in NiO," Phys. Rev. Lett. 121, 097402.

Tancogne-Dejean, N., M. A. Sentef, and A. Rubio, 2020, "Ultrafast transient absorption spectroscopy of the charge-transfer insulator NiO: Beyond the dynamical Franz-Keldysh effect," Phys. Rev. B 102, 115106.

Täuber, U.C., 2017, "Phase transitions and scaling in systems far from equilibrium," Annu. Rev. Condens. Matter Phys. 8, 185.

Teitelbaum, S. W., B. K. Ofori-Okai, Y. H. Cheng, J. Zhang, F. Jin, W. Wu, R. D. Averitt, and K. A. Nelson, 2019, "Dynamics of a Persistent Insulator-to-Metal Transition in Strained Manganite Films," Phys. Rev. Lett. 123, 267201.

Teitelbaum, S. W., T. Shin, J. W. Wolfson, Y. H. Cheng, I. J. Porter, M. Kandyla, and K. A. Nelson, 2018, "Real-Time Observation of a Coherent Lattice Transformation into a High-Symmetry Phase," Phys. Rev. X 8, 031081.

Terada, Y., S. Yoshida, O. Takeuchi, and H. Shigekawa, 2010, "Realspace imaging of transient carrier dynamics by nanoscale pumpprobe microscopy," Nat. Photonics 4, 869. 
Thygesen, K. S., and A. Rubio, 2007, "Nonequilibrium $G W$ approach to quantum transport in nano-scale contacts," J. Chem. Phys. 126, 091101.

Tindall, J. and B. Buča, J. R. Coulthard, and D. Jaksch, 2019, "Heating-Induced Long-Range $\eta$ Pairing in the Hubbard Model," Phys. Rev. Lett. 123, 030603.

Tindall, J., F. Schlawin, M. Buzzi, D. Nicoletti, J. R. Coulthard, H. Gao, A. Cavalleri, M. A. Sentef, and D. Jaksch, 2020, "Dynamical Order and Superconductivity in a Frustrated Many-Body System," Phys. Rev. Lett. 125, 137001.

Tokura, Y., 2006, "Photoinduced phase transition: A tool for generating a hidden state of matter," J. Phys. Soc. Jpn. 75, 011001.

Tokura, Y., M. Kawasaki, and N. Nagaosa, 2017, "Emergent functions of quantum materials," Nat. Phys. 13, 1056.

Tokura, Y., and N. Nagaosa, 2000, "Orbital physics in transitionmetal oxides," Science 288, 462.

Topp, G. E., G. Jotzu, J. W. McIver, L. Xian, A. Rubio, and M. A. Sentef, 2019, "Topological Floquet engineering of twisted bilayer graphene,” Phys. Rev. Research 1, 023031.

Torchinsky, D. H., F. Mahmood, A. T. Bollinger, I. Božović, and N. Gedik, 2013, "Fluctuating charge-density waves in a cuprate superconductor," Nat. Mater. 12, 387.

Trigo, M., J. Chen, M. P. Jiang, W. L. Mao, S. C. Riggs, M. C. Shapiro, I. R. Fisher, and D. A. Reis, 2012, "Ultrafast pump-probe measurements of short small-polaron lifetimes in the mixedvalence perovskite $\mathrm{Cs}_{2} \mathrm{Au}_{2} \mathrm{I}_{6}$ under high pressures," Phys. Rev. B 85, 081102.

Trigo, M., et al., 2013, "Fourier-transform inelastic x-ray scattering from time- and momentum-dependent phonon-phonon correlations," Nat. Phys. 9, 790.

Tsen, Kong-Thon, 2001, Ultrafast Phenomena in Semiconductors (Springer, New York).

Tsuji, N., and H. Aoki, 2015, "Theory of Anderson pseudospin resonance with Higgs mode in superconductors," Phys. Rev. B 92, 064508.

Tsuji, N., M. Eckstein, and P. Werner, 2013, "Nonthermal Antiferromagnetic Order and Nonequilibrium Criticality in the Hubbard Model," Phys. Rev. Lett. 110, 136404.

Tsuji, N., T. Oka, and H. Aoki, 2008, "Correlated electron systems periodically driven out of equilibrium: Floquet + DMFT formalism," Phys. Rev. B 78, 235124.

Ullrich, C., 2012, Time-Dependent Density-Functional Theory: Concepts and Applications (Oxford University Press, New York).

Usaj, G., P. M. Perez-Piskunow, L. E. F. Foa Torres, and C. A. Balseiro, 2014, "Irradiated graphene as a tunable Floquet topological insulator," Phys. Rev. B 90, 115423.

Vajna, S., K. Klobas, T. Prosen, and A. Polkovnikov, 2018, "Replica Resummation of the Baker-Campbell-Hausdorff Series," Phys. Rev. Lett. 120, 200607.

Vaskivskyi, I., J. Gospodaric, S. Brazovskii, D. Svetin, P. Sutar, E. Goreshnik, I. A. Mihailovic, T. Mertelj, and D. Mihailovic, 2015, "Controlling the metal-to-insulator relaxation of the metastable hidden quantum state in $1 T-\mathrm{TaS}_{2}, "$ Sci. Adv. 1, e1500168.

Verstraete, F., V. Murg, and J.I. Cirac, 2008, "Matrix product states, projected entangled pair states, and variational renormalization group methods for quantum spin systems," Adv. Phys. 57, 143.

Vodeb, J., V. V. Kabanov, Y. A. Gerasimenko, I. Vaskivskyi, J. Ravnik, and D. Mihailovic, 2019, "Theoretical modeling of the non-equilibrium amorphous state in $1 T-\mathrm{TaS}_{2}$," J. Supercond. Novel Magn. 32, 3057.
Vogelgesang, S., G. Storeck, J. G. Horstmann, T. Diekmann, M. Sivis, S. Schramm, Rossnagel K., S. Schäfer, and C. Ropers, 2018, "Phase ordering of charge density waves traced by ultrafast lowenergy electron diffraction," Nat. Phys. 14, 184.

von Hoegen, A., R. Mankowsky, M. Fechner, M. Först, and A. Cavalleri, 2018, "Probing the interatomic potential of solids with strong-field nonlinear phononics," Nature (London) 555, 79.

Wagner, M., et al., 2014, "Ultrafast and nanoscale plasmonic phenomena in exfoliated graphene revealed by infrared pumpprobe nanoscopy," Nano Lett. 14, 894.

Waldecker, L., R. Bertoni, R. Ernstorfer, and J. Vorberger, 2016, "Electron-Phonon Coupling and Energy Flow in a Simple Metal beyond the Two-Temperature Approximation," Phys. Rev. X 6, 021003.

Waldecker, L., R. Bertoni, H. Hübener, T. Brumme, T. Vasileiadis, D. Zahn, A. Rubio, and R. Ernstorfer, 2017, "Momentum-Resolved View of Electron-Phonon Coupling in Multilayer WSe $\mathrm{W}_{2}$," Phys. Rev. Lett. 119, 036803

Wall, S., et al., 2018, "Ultrafast disordering of vanadium dimers in photoexcited $\mathrm{VO}_{2}$, , Science 362, 572.

Walldorf, N., D. M. Kennes, J. Paaske, and A. J. Millis, 2019, "The antiferromagnetic phase of the Floquet-driven Hubbard model," Phys. Rev. B 100, 121110.

Walowski, J., and M. Münzenberg, 2016, "Ultrafast magnetism and THz spintronics,” J. Appl. Phys. 120, 140901.

Wang, J., and S.-C. Zhang, 2017, "Topological states of condensed matter," Nat. Mater. 16, 1062.

Wang, Y., C.-C. Chen, B. Moritz, and T. P. Devereaux, 2018, "LightEnhanced Spin Fluctuations and $d$-Wave Superconductivity at a Phase Boundary," Phys. Rev. Lett. 120, 246402.

Wang, Y., M. Claassen, C. D. Pemmaraju, C. Jia, B. Moritz, and T. P. Devereaux, 2018, "Theoretical understanding of photon spectroscopies in correlated materials in and out of equilibrium," Nat. Rev. Mater. 3, 312.

Wang, Y., T. P. Devereaux, and C.-C. Chen, 2018, "Theory of timeresolved Raman scattering in correlated systems: Ultrafast engineering of spin dynamics and detection of thermalization," Phys. Rev. B 98, 245106.

Wang, Y. H., H. Steinberg, P. Jarillo-Herrero, and N. Gedik, 2013, "Observation of Floquet-Bloch states on the surface of a topological insulator," Science 342, 453.

Weathersby, S. P., et al., 2015, "Mega-electron-volt ultrafast electron diffraction at SLAC National Accelerator Laboratory," Rev. Sci. Instrum. 86, 073702.

Wegkamp, D., et al., 2014, "Instantaneous Band Gap Collapse in Photoexcited Monoclinic $\mathrm{VO}_{2}$ due to Photocarrier Doping," Phys. Rev. Lett. 113, 216401.

Weidinger, S., and M. Knap, 2017, "Floquet prethermalization and regimes of heating in a periodically driven, interacting quantum system," Sci. Rep. 7, 45382.

Werdehausen, D., T. Takayama, M. Höppner, G. Albrecht, A. W. Rost, Y. Lu, D. Manske, H. Takagi, and S. Kaiser, 2018, "Coherent order parameter oscillations in the ground state of the excitonic insulator $\mathrm{Ta}_{2} \mathrm{NiSe}_{5}, "$ Sci. Adv. 4, eaap8652.

Werner, P., and Y. Murakami, 2020, "Nonthermal excitonic condensation near a spin-state transition," Phys. Rev. B 102, 241103.

Werner, P., T. Oka, and A. J. Millis, 2009, "Diagrammatic Monte Carlo simulation of nonequilibrium systems," Phys. Rev. B 79, 035320.

Werner, P., N. Tsuji, and M. Eckstein, 2012, "Nonthermal symmetrybroken states in the strongly interacting Hubbard model," Phys. Rev. B 86, 205101. 
Woerner, M., W. Kuehn, P. Bowlan, K. Reimann, and T. Elsaesser, 2013, "Ultrafast two-dimensional terahertz spectroscopy of elementary excitations in solids," New J. Phys. 15, 025039.

Yang, L.X., et al., 2014, "Ultrafast Modulation of the Chemical Potential in $\mathrm{BaFe}_{2} \mathrm{As}_{2}$ by Coherent Phonons," Phys. Rev. Lett. 112, 207001.

Yang, S.-L., J. A. Sobota, Y. He, D. Leuenberger, H. Soifer, H. Eisaki, P. S. Kirchmann, and Z.-X. Shen, 2019, "Mode-Selective Coupling of Coherent Phonons to the Bi2212 Electronic Band Structure," Phys. Rev. Lett. 122, 176403.

Yoshida, S., Y. Aizawa, Z.-H. Wang, R. Oshima, Y. Mera, E. Matsuyama, H. Oigawa, O. Takeuchi, and H. Shigekawa, 2014, "Probing ultrafast spin dynamics with optical pump-probe scanning tunnelling microscopy," Nat. Nanotechnol. 9, 588.

Yu, G., C. H. Lee, A. J. Heeger, N. Herron, and E. M. McCarron, 1991, "Transient Photoinduced Conductivity in Single Crystals of $\mathrm{YBa}_{2} \mathrm{Cu}_{3} \mathrm{O}_{6.3}$ : 'Photodoping' to the Metallic State," Phys. Rev. Lett. 67, 2581.

Yu, T., M. Claassen, D. M. Kennes, and M. A. Sentef, 2021, "Optical manipulation of domains in chiral topological superconductors," Phys. Rev. Research 3, 013253.

Yusupov, R., T. Mertelj, S. Kabanov, V. V. and Brazovskii, P. Kusar, J.-H. Chu, I. R. Fisher, and D. Mihailovic, 2010, "Coherent dynamics of macroscopic electronic order through a symmetry breaking transition," Nat. Phys. 6, 681.

Yusupov, R. V., D. Mihailovic, C. V. Colin, G. R. Blake, and T. T. M. Palstra, 2010, "Critical phenomena and femtosecond ordering dynamics associated with electronic and spin-ordered phases in $\mathrm{YVO}_{3}$ and $\mathrm{GdVO}_{3}$," Phys. Rev. B 81, 075103.

Zeng, T.-S., and D. N. Sheng, 2017, "Prethermal time crystals in a one-dimensional periodically driven Floquet system," Phys. Rev. B 96, 094202.

Zewail, A. H., 2006, "4D ultrafast electron diffraction, crystallography, and microscopy,” Annu. Rev. Phys. Chem. 57, 65.

Zhang, J., and R.D. Averitt, 2014, "Dynamics and control in complex transition metal oxides," Annu. Rev. Mater. Res. 44, 19.

Zhang, J., X. Tan, M. Liu, S. W. Teitelbaum, K. W. Post, Feng Jin, K. A. Nelson, D. N. Basov, Wenbin Wu, and R. D. Averitt, 2016, "Cooperative photoinduced metastable phase control in strained manganite films," Nat. Mater. 15, 956.
Zhang, P., et al., 2017, “Topologically Entangled Rashba-Split Shockley States on the Surface of Grey Arsenic," Phys. Rev. Lett. 118, 046802.

Zhang, S. J., Z. X. Wang, H. Xiang, X. Yao, Q. M. Liu, L. Y. Shi, T. Lin, T. Dong, D. Wu, and N. L. Wang, 2020, "Photoinduced Nonequilibrium Response in Underdoped $\mathrm{YBa}_{2} \mathrm{Cu}_{3} \mathrm{O}_{6+x}$ Probed by Time-Resolved Terahertz Spectroscopy," Phys. Rev. X 10, 011056.

Zhao, L., et al., 2018, “Terahertz Streaking of Few-Femtosecond Relativistic Electron Beams," Phys. Rev. X 8, 021061.

Zhong, Z., N. M. Gabor, J.E. Sharping, A. L. Gaeta, and P. L. McEuen, 2008, "Terahertz time-domain measurement of ballistic electron resonance in a single-walled carbon nanotube," Nat. Nanotechnol. 3, 201.

Zhou, F., J. Williams, S. Sun, C. D. Malliakas, M. G. Kanatzidis, A. F. Kemper, and C.-Y. Ruan, 2021, "Nonequilibrium dynamics of spontaneous symmetry breaking into a hidden state of chargedensity wave," Nat. Commun. 12, 566.

Zhou, X., S. He, G. Liu, L. Zhao, L. Yu, and W. Zhang, 2018, "New developments in laser-based photoemission spectroscopy and its scientific applications: A key issues review," Rep. Prog. Phys. 81, 062101.

Zhu, D., A. Robert, T. Henighan, H. T. Lemke, M. Chollet, J. M. Glownia, D. A. Reis, and M. Trigo, 2015, "Phonon spectroscopy with sub-meV resolution by femtosecond x-ray diffuse scattering," Phys. Rev. B 92, 054303.

Zhu, Y., J. Hoffman, C. E. Rowland, H. Park, D. A. Walko, J. W. Freeland, P. J. Ryan, R. D. Schaller, A. Bhattacharya, and H. Wen, 2018, "Unconventional slowing down of electronic recovery in photoexcited charge-ordered $\mathrm{La}_{1 / 3} \mathrm{Sr}_{2 / 3} \mathrm{FeO}_{3}$," Nat. Commun. 9, 1799.

Zong, A., et al., 2018, "Ultrafast manipulation of mirror domain walls in a charge density wave," Sci. Adv. 4, eaau5501.

Zong, A., et al., 2019a, "Dynamical Slowing-Down in an Ultrafast Photoinduced Phase Transition,” Phys. Rev. Lett. 123, 097601.

Zong, A., et al., 2019b, "Evidence for topological defects in a photoinduced phase transition," Nat. Phys. 15, 27.

Zurek, W. H., 1996, "Cosmological experiments in condensed matter systems,” Phys. Rep. 276, 177. 